\title{
LYAPUNOV SPECTRUM OF ASYMPTOTICALLY SUB-ADDITIVE POTENTIALS
}

\author{
DE-JUN FENG AND WEN HUANG
}

\begin{abstract}
For general asymptotically sub-additive potentials (resp. asymptotically additive potentials) on general topological dynamical systems, we establish some variational relations between the topological entropy of the level sets of Lyapunov exponents, measuretheoretic entropies and topological pressures in this general situation. Most of our results are obtained without the assumption of the existence of unique equilibrium measures or the differentiability of pressure functions. Some examples are constructed to illustrate the irregularity and the complexity of multifractal behaviors in the sub-additive case and in the case that the entropy map that is not upper-semi continuous.
\end{abstract}

\section{INTRODUCTION}

The present paper is devoted to the study of the multifractal behavior of Lyapunov exponents of asymptotically sub-additive potentials. This is mainly motivated by the recent works on the Lyapunov exponents of matrix products [26, 23, 24] and the Lyapunov exponents of differential maps on nonconformal repellers [6].

Before formulating our results, we first give some notation and backgrounds. We call $(X, T)$ a topological dynamical system (for short TDS) if $X$ is a compact metric space and $T$ is a continuous map from $X$ to $X$. A sequence $\Phi=\left\{\log \phi_{n}\right\}_{n=1}^{\infty}$ of functions on $X$ is said to be a sub-additive potential if each $\phi_{n}$ is a continuous nonnegative-valued function on $X$ such that

$$
0 \leq \phi_{n+m}(x) \leq \phi_{n}(x) \phi_{m}\left(T^{n} x\right), \quad \forall x \in X, m, n \in \mathbb{N} .
$$

More generally, $\Phi=\left\{\log \phi_{n}\right\}_{n=1}^{\infty}$ is said to be an asymptotically sub-additive potential if for any $\epsilon>0$, there exists a sub-additive potential $\Psi=\left\{\log \psi_{n}\right\}_{n=1}^{\infty}$ on $X$ such that

$$
\limsup _{n \rightarrow \infty} \frac{1}{n} \sup _{x \in X}\left|\log \phi_{n}(x)-\log \psi_{n}(x)\right| \leq \epsilon
$$

where we take the convention $\log 0-\log 0=0$. Furthermore $\Phi$ is called an asymptotically additive potential if both $\Phi$ and $-\Phi$ are asymptotically sub-additive, where $-\Phi$ denotes $\left\{\log \left(1 / \phi_{n}\right)\right\}_{n=1}^{\infty}$. In particular, $\Phi$ is called additive if each $\phi_{n}$ is a continuous positive-valued function so that $\phi_{n+m}(x)=\phi_{n}(x) \phi_{m}\left(T^{n} x\right)$ for all $x \in X$ and $m, n \in \mathbb{N}$; in this case, there is a continuous real function $g$ on $X$ such that $\phi_{n}(x)=\exp \left(\sum_{i=0}^{n-1} g\left(T^{i} x\right)\right)$ for each $n$.

Key words and phrases. Lyapunov exponents, Multifractal analysis, Variational principle.

2000 Mathematics Subject Classification: 37D35; 37C45. 
Let $\Phi=\left\{\log \phi_{n}\right\}_{n=1}^{\infty}$ be an asymptotically sub-additive potential on $X$. For any $x \in X$, we define

$$
\lambda_{\Phi}(x)=\lim _{n \rightarrow \infty} \frac{\log \phi_{n}(x)}{n}
$$

and call it the Lyapunov exponent of $\Phi$ at $x$, provided that the limit exists. Otherwise we use $\bar{\lambda}_{\Phi}(x)$ and $\underline{\lambda}_{\Phi}(x)$ to denote the upper and lower limits respectively. It can be derived from Kingman's sub-additive ergodic theorem (cf. [49], p. 231) that, for any $\mu \in \mathcal{E}(X, T)$,

$$
\lambda_{\Phi}(x)=\Phi_{*}(\mu) \quad \text { for } \mu \text {-a.e. } x \in X,
$$

where $\mathcal{E}(X, T)$ denotes the space of ergodic $T$-invariant Borel probability measures on $X$ and

$$
\Phi_{*}(\mu):=\lim _{n \rightarrow \infty} \int \frac{\log \phi_{n}(x)}{n} d \mu(x) .
$$

This limit always exists and takes values in $\mathbb{R} \cup\{-\infty\}$. (For details, see Proposition A.1.)

In this paper we are mainly concerned with the distribution of the Lyapunov exponents of $\Phi$. More precisely, for any $\alpha \in \mathbb{R}$, define

$$
E_{\Phi}(\alpha)=\left\{x \in X: \lambda_{\Phi}(x)=\alpha\right\},
$$

which is called the $\alpha$-level set of $\lambda_{\Phi}$. We shall study the topological entropy $h_{\text {top }}\left(T, E_{\Phi}(\alpha)\right)$ of $E_{\Phi}(\alpha)$ when $\alpha$ varies (here we are using the notion of topological entropy for arbitrary subsets of a compact space, introduced by Bowen in [12]; see Section 2.1). This is a general concept of multifractal analysis proposed by Barreira, Pesin and Schmeling [7], and it plays an important role in the dimension theory of dynamical systems [43, 5]. For convenience we call $h_{\mathrm{top}}\left(T, E_{\Phi}(\alpha)\right)$, as a function of $\alpha$, the Lyapunov spectrum of $\Phi$.

A key ingredient in the above study is the topological pressure of $\Phi$. To introduce this concept, let $X$ be endowed with the metric $d$. For any $n \in \mathbb{N}$, define a new metric $d_{n}$ on $X$ by

$$
d_{n}(x, y)=\max \left\{d\left(T^{k}(x), T^{k}(y)\right): k=0, \ldots, n-1\right\} .
$$

For any $\epsilon>0$, a set $E \subseteq X$ is said to be a $(n, \epsilon)$-separated subset of $X$ if $d_{n}(x, y)>\epsilon$ for any two different points $x, y \in E$. For $\Phi=\left\{\log \phi_{n}\right\}_{n=1}^{\infty}$, we define

$$
P_{n}(T, \Phi, \epsilon)=\sup \left\{\sum_{x \in E} \phi_{n}(x): E \text { is a }(n, \epsilon) \text {-separated subset of } X\right\} \text {. }
$$

It is clear that $P_{n}(T, \Phi, \epsilon)$ is a decreasing function of $\epsilon$. Define

$$
P(T, \Phi, \epsilon)=\limsup _{n \rightarrow \infty} \frac{1}{n} \log P_{n}(T, \Phi, \epsilon)
$$

and $P(T, \Phi)=\lim _{\epsilon \rightarrow 0} P(T, \Phi, \epsilon)$. We call $P(T, \Phi)$ the topological pressure of $\Phi$ with respect to $T$ or, simply, the topological pressure of $\Phi$. If $\Phi$ is additive, $P(T, \Phi)$ recovers the classical (additive) topological pressure introduced by Ruelle and Walters (cf. [49, Chapter 9]). 
Let us return back to the study of the Lyapunov spectrum. When $\Phi=\left\{\sum_{i=0}^{n-1} f \circ\right.$ $\left.T^{i}\right\}_{n=1}^{\infty}$ is an additive potential, the Lyapunov exponent $\lambda_{\Phi}$ is just equal to the Birkhoff average of $f$. In this case, the topological entropy (or the Hausdorff dimension) of the level sets of Birkhoff averages has been extensively studied in the recent two decades (see, e.g., [13, 7, 41, 9, 20, 21, 34, 27, 8, 42, 47, 23, 16, 22, 1, 2, 29] and references therein). It is well known (see, e.g. [21, 23, 41]) that when $(X, T)$ is a transitive subshift of finite type and $\Phi$ is an additive potential, then

$$
E_{\Phi}(\alpha) \neq \emptyset \Longleftrightarrow \alpha \in \Omega:=\left\{\Phi_{*}(\mu): \mu \in \mathcal{M}(X, T)\right\},
$$

where $\mathcal{M}(X, T)$ denotes the space of $T$-invariant Borel probability measures on $X$ and

$$
\begin{aligned}
h_{\text {top }}\left(T, E_{\Phi}(\alpha)\right) & =\sup \left\{h_{\mu}(T): \mu \in \mathcal{M}(X, T) \text { with } \Phi_{*}(\mu)=\alpha\right\} \\
& =\inf \left\{P_{\Phi}(q)-\alpha q: q \in \mathbb{R}\right\}, \quad \forall \alpha \in \Omega .
\end{aligned}
$$

where $h_{\mu}(T)$ denotes the measure-theoretic entropy of $\mu, P_{\Phi}(q):=P(T, q \Phi)$ and $q \Phi$ denotes the potential $\left\{q \log \phi_{n}\right\}_{n=1}^{\infty}$. The first variational relation in (1.7) has been extended to any TDS satisfying the specification property [47].

Motivated by the study of the multifractal formalism associated to certain iterated function systems with overlaps, the Lyapunov spectrum of certain special sub-additive potentials $\Phi=\left\{\log \phi_{n}\right\}_{n=1}^{\infty}$ on subshifts of finite type have been studied in [26, 23, 24], in which $\phi_{n}(x)=\left\|\prod_{i=0}^{n-1} M\left(T^{i} x\right)\right\|$, where $M$ is a continuous function on $X$ taking values in the set of $d \times d$ matrices, and $\|\cdot\|$ denotes the operator norm. It is known that in this general situation, (1.6) and (1.7) may both fail. The following is an example taken from [24].

Example 1.1 Let $(X, T)$ be the one-sided full shift over the alphabet $\{1,2,3,4\}$. Let $M(x)$ be a matrix function on $X$ defined as $M(x)=M_{x_{1}}$ for $x=\left(x_{j}\right)_{j=1}^{\infty}$, where $M_{i}(1 \leq i \leq 4)$ are diagonal $4 \times 4$ matrices given by

$$
M_{1}=M_{2}=\operatorname{diag}(1,2,0,0), \quad M_{3}=\operatorname{diag}(1,0,3,0), M_{4}=\operatorname{diag}(1,0,0,4) .
$$

It is easily checked that

and

$$
P_{\Phi}(q)= \begin{cases}q \log 4, & \text { if } q \geq 1 \\ \log 4, & \text { otherwise }\end{cases}
$$

$$
\begin{aligned}
\left\{\alpha \in \mathbb{R}: E_{\Phi}(\alpha) \neq \emptyset\right\} & =\{0, \log 2, \log 3, \log 4\} \\
& \varsubsetneqq[0, \log 4]=\left\{\alpha \in \mathbb{R}: \Phi_{*}(\mu)=\alpha \text { for some } \mu \in \mathcal{M}(X, T)\right\} .
\end{aligned}
$$

Furthermore, $E_{\Phi}(\log 3)$ is a singleton and thus

$$
h_{\mathrm{top}}\left(T, E_{\Phi}(\log 3)\right)=0<\log 4-\log 3=\inf _{q \in \mathbb{R}}\left\{-q \log 3+P_{\Phi}(q)\right\} .
$$

We remark that under some additional assumptions (e.g., positiveness or certain irreducibility) for the matrix function $M,(1.6)$ and (1.7) still hold [26, 23, 24. A natural question arises whether there exist some positive results without any additional assumptions. This is one of the original motivations of this paper. 
Indeed in this paper, we study the Lyapunov spectrum of general asymptotically subadditive potentials and asymptotically additive potentials on general TDS. Under this setting, the multifractal behavior may be quite irregular. For instance, we can construct a $\operatorname{TDS}(X, T)$ and an additive potential $\Phi$ on $X$ such that

$$
h_{\text {top }}\left(T, E_{\Phi}(\alpha)\right)<\inf \left\{P_{\Phi}(q)-\alpha q: q \in \mathbb{R}\right\} \quad \forall \alpha \in \Omega:=\left\{\Phi_{*}(\mu): \mu \in \mathcal{M}(X, T)\right\} .
$$

(See Example 6.2). Nevertheless, we still have some positive results regarding the Lyapunov spectrum and its variational relations to measure-theoretic entropies and topological pressures. Some more properties are obtained when the corresponding TDS satisfies further assumptions (e.g., upper semi-continuity of the entropy map).

To formulate our results, for an asymptotically sub-additive potential $\Phi=\left\{\log \phi_{n}\right\}$ on a general TDS $(X, T)$, we define

$$
\bar{\beta}(\Phi)=\lim _{n \rightarrow \infty} \frac{1}{n} \log \sup _{x \in X} \phi_{n}(x) .
$$

The limit exists and takes values in $\mathbb{R} \cup\{-\infty\}$ (see Lemma A.3). However if $\bar{\beta}(\Phi)=-\infty$, it is easy to see that for all $x \in X, \lambda_{\Phi}(x)=-\infty$. To avoid trivialities we shall always assume that $\bar{\beta}(\Phi)>-\infty$. For any $q>0$, let $q \Phi$ denote the sequence $\left\{q \log \phi_{n}\right\}_{i=1}^{\infty}$ (which clearly is asymptotically sub-additive) and write

$$
P_{\Phi}(q)=P(T, q \Phi) \text {. }
$$

The function $P_{\Phi}$ is called the pressure function of $\Phi$. When $\Phi$ is asymptotically additive on $X, P_{\Phi}$ can be defined over $(-\infty, \infty)$.

Our main results are Theorems 1.1,1.4 formulated as follows:

Theorem 1.1. Let $(X, T)$ be a TDS and $\Phi=\left\{\log \phi_{n}\right\}_{n=1}^{\infty}$ an asymptotically sub-additive potential on $X$ which satisfies $\bar{\beta}(\Phi)>-\infty$. Then $E_{\Phi}(\bar{\beta}(\Phi)) \neq \emptyset$ and

$$
\begin{aligned}
h_{\mathrm{top}}\left(T, E_{\Phi}(\bar{\beta}(\Phi))\right) & =\sup \left\{h_{\mu}(T): \mu \in \mathcal{M}(X, T), \Phi_{*}(\mu)=\bar{\beta}(\Phi)\right\} \\
& =\sup \left\{h_{\mu}(T): \mu \in \mathcal{E}(X, T), \Phi_{*}(\mu)=\bar{\beta}(\Phi)\right\} .
\end{aligned}
$$

We emphasize that the above theorem only deals with the specific value $\alpha=\bar{\beta}(\Phi)$, which is the largest possible value for $\lambda_{\Phi}$ (cf. Lemma A.3).

Theorem 1.2. Let $(X, T)$ be a TDS such that the topological entropy $h_{\mathrm{top}}(T)$ is finite. Suppose that $\Phi=\left\{\log \phi_{n}\right\}_{n=1}^{\infty}$ is an asymptotically sub-additive potential on $X$ which satisfies $\bar{\beta}(\Phi)>-\infty$. Then the pressure function $P_{\Phi}(q)$ is a continuous real convex function on $(0, \infty)$ with $P_{\Phi}^{\prime}(\infty):=\lim _{q \rightarrow \infty} P_{\Phi}(q) / q=\bar{\beta}(\Phi)$. Moreover,

(i) For any $t>0$, if $\alpha=P_{\Phi}^{\prime}(t+)$ or $\alpha=P_{\Phi}^{\prime}(t-)$, then

$$
\lim _{\epsilon \rightarrow 0} h_{\text {top }}\left(T, \underset{\beta \in(\alpha-\epsilon, \alpha+\epsilon)}{\bigcup} E_{\Phi}(\beta)\right)=\inf _{q>0}\left\{P_{\Phi}(q)-\alpha q\right\}=P_{\Phi}(t)-\alpha t,
$$


where $P_{\Phi}^{\prime}(t-)$ and $P_{\Phi}^{\prime}(t+)$ denote the left and right derivatives of $P_{\Phi}$ at $t$, respectively. Moreover the first equality is also valid when $\alpha=P_{\Phi}^{\prime}(\infty)$.

(ii) For any $t>0$ and any $\alpha \in\left[P_{\Phi}^{\prime}(t-), P_{\Phi}^{\prime}(t+)\right]$,

$$
\inf _{q>0}\left\{P_{\Phi}(q)-\alpha q\right\}=\lim _{\epsilon \rightarrow 0} \sup \left\{h_{\mu}(T): \mu \in \mathcal{M}(X, T),\left|\Phi_{*}(\mu)-\alpha\right|<\epsilon\right\} .
$$

Furthermore the above equality is valid for $\alpha=P_{\Phi}^{\prime}(\infty)$.

(iii) For any $\alpha \in\left(\lim _{t \rightarrow 0+} P_{\Phi}^{\prime}(t-), P_{\Phi}^{\prime}(\infty)\right)$,

$$
\inf _{q>0}\left\{P_{\Phi}(q)-\alpha q\right\}=\sup \left\{h_{\mu}(T): \mu \in \mathcal{M}(X, T), \Phi_{*}(\mu)=\alpha\right\} .
$$

By convexity, $P_{\Phi}$ may fail to be differentiable on a set which is at most countable, however the left and right derivatives of $P_{\Phi}$ exist everywhere. We remark that $h_{\text {top }}(T)$ is finite for a lot of TDS's such as expansive maps on compact metric space and Lipschitz continuous transformations on finite dimensional compact metric spaces (see e.g. [32, Section 3.2]), and asymptotically $h$-expansive TDS's [39]. We need to mention that Theorem 1.2(i) only deals with the "fuzzy" level sets and it is not valid for the standard level sets $E_{\Phi}(\alpha)$. Indeed, there are examples such that

$$
h_{\text {top }}\left(T, E_{\Phi}(\alpha)\right)<\inf _{q>0}\left\{P_{\Phi}(q)-\alpha q\right\}
$$

for any $\alpha=P_{\Phi}^{\prime}(t+)$ or $\alpha=P_{\Phi}^{\prime}(t-)$ with $t>0$ (see e.g. Example 6.2). Nevertheless the results of Theorem 1.2 can be improved if we add an additional assumption that the entropy map $\mu \rightarrow h_{\mu}(T)$ is upper semi-continuous on $\mathcal{M}(X, T)$. More precisely, we have

Theorem 1.3. Under the condition of Theorem 1.2, we assume furthermore that the entropy map $\mu \rightarrow h_{\mu}(T)$ is upper semi-continuous on $\mathcal{M}(X, T)$. Then

(i) For any $t>0$, if $\alpha=P_{\Phi}^{\prime}(t+)$ or $\alpha=P_{\Phi}^{\prime}(t-)$, then $E_{\Phi}(\alpha) \neq \emptyset$ and

$$
h_{\mathrm{top}}\left(T, E_{\Phi}(\alpha)\right)=\inf _{q>0}\left\{P_{\Phi}(q)-\alpha q\right\}=P_{\Phi}(t)-\alpha t
$$

(ii) For $\alpha \in \bigcup_{t>0}\left[P_{\Phi}^{\prime}(t-), P_{\Phi}^{\prime}(\infty)\right]$,

$$
\inf _{q>0}\left\{P_{\Phi}(q)-\alpha q\right\}=\max \left\{h_{\mu}(T): \mu \in \mathcal{M}(X, T), \Phi_{*}(\mu)=\alpha\right\} .
$$

(iii) If $t>0$ such that $t \Phi$ has a unique equilibrium state $\mu_{t} \in \mathcal{M}(X, T)$, then $\mu_{t}$ is ergodic, $P_{\Phi}^{\prime}(t)=\Phi_{*}\left(\mu_{t}\right), E_{\Phi}\left(P_{\Phi}^{\prime}(t)\right) \neq \emptyset$ and $h_{\mathrm{top}}\left(T, E_{\Phi}\left(P_{\Phi}^{\prime}(t)\right)\right)=h_{\mu_{t}}(T)$.

A significant part of Theorem 1.3(i) is that we don't need the differentiability assumption for $P_{\Phi}$. To the best of our knowledge, this result is not known even in the additive case. It has a nice application in the multifractal analysis for certain probability measures on symbolic spaces (see Remark 4.9). We remark that the assumption of upper semi-continuity for the entropy map is quite essential for the results in Theorem 1.3. This assumption is satisfied by some natural TDS's such as $h$-expansive TDS's [1] and more generally, asymptotically $h$-expansive TDS's [39] which include, for example, $C^{\infty}$ transformations on Riemannian manifolds [14]. Without this assumption, the multifractal behavior may be 
very irregular and complicated. See Section 6 for some examples. We remark that the differentiability property of the pressure functions was studied in [37, 38, for rational maps on the Riemann sphere for certain additive potentials.

Meanwhile Theorems 1.1 1.3 are about asymptotically sub-additive potentials, our next theorem is concerned with asymptotically additive potentials. A TDS $(X, T)$ is called to be saturated if for any $\mu \in \mathcal{M}(X, T)$, we have $G_{\mu} \neq \emptyset$ and $h_{\text {top }}\left(T, G_{\mu}\right)=h_{\mu}(T)$, where $G_{\mu}$ denotes the set of $\mu$-generic points defined by

$$
G_{\mu}:=\left\{x \in X: \frac{1}{n} \sum_{j=0}^{n-1} \delta_{T^{j} x} \rightarrow \mu \text { in the weak* topology as } n \rightarrow \infty\right\},
$$

where $\delta_{y}$ denotes the probability measure whose support is the single point $y$. It was shown independently in [22, 44] that if a TDS $(X, T)$ satisfies the specification property, then $(X, T)$ is saturated.

Theorem 1.4. Let $(X, T)$ be a TDS and let $\Phi$ be an asymptotically additive potential on $X$. Set $\Omega=\left\{\Phi_{*}(\mu): \mu \in \mathcal{M}(X, T)\right\}$. Then $\Omega$ is a bounded closed interval. Furthermore we have the following statements.

(i) $\left\{\alpha \in \mathbb{R}: E_{\Phi}(\alpha) \neq \emptyset\right\} \subseteq \Omega$.

(ii) If $h_{\text {top }}(T)<\infty$, then $P_{\Phi}$ is a real convex function over $\mathbb{R}$. Furthermore, $\alpha \in \Omega \Longleftrightarrow \inf \left\{P_{\Phi}(q)-\alpha q: q \in \mathbb{R}\right\} \neq-\infty \Longleftrightarrow \inf \left\{P_{\Phi}(q)-\alpha q: q \in \mathbb{R}\right\} \geq 0$.

(iii) If $h_{\mathrm{top}}(T)<\infty$ and the entropy map is upper semi-continuous, then for each $\alpha \in \Omega$,

$$
\sup \left\{h_{\mu}(T): \mu \in \mathcal{M}(X, T), \Phi_{*}(\mu)=\alpha\right\}=\inf \left\{P_{\Phi}(q)-\alpha q: q \in \mathbb{R}\right\} ;
$$

Furthermore, for $\alpha \in \bigcup_{t \in \mathbb{R}}\left\{P_{\Phi}^{\prime}(t-), P_{\Phi}^{\prime}(t+)\right\} \cup P_{\Phi}^{\prime}( \pm \infty)$, we have $E_{\Phi}(\alpha) \neq \emptyset$ and

$$
h_{\text {top }}\left(T, E_{\Phi}(\alpha)\right)=\inf \left\{P_{\Phi}(q)-\alpha q: q \in \mathbb{R}\right\},
$$

where $P_{\Phi}^{\prime}(+\infty):=\lim _{q \rightarrow+\infty} P_{\Phi}(q) / q$ and $P_{\Phi}^{\prime}(-\infty):=\lim _{q \rightarrow-\infty} P_{\Phi}(q) / q$.

(iv) Assume that $(X, T)$ is saturated. Then $E_{\Phi}(\alpha) \neq \emptyset$ if and only if $\alpha \in \Omega$. Furthermore, $h_{\mathrm{top}}\left(T, E_{\Phi}(\alpha)\right)=\sup \left\{h_{\mu}(T): \mu \in \mathcal{M}(X, T), \Phi_{*}(\mu)=\alpha\right\}$ for any $\alpha \in \Omega$.

We remark that Theorem 1.4(iii)-(iv) extend previous results about the Lyapunov spectrum of continuous positive matrix-valued functions [23] and the Lyapunov spectrum of certain asymptotically additive potentials [35] on subshifts of finite type.

In this paper, we also study the high dimensional Lyapunov spectrum. For a finite family of asymptotically sub-additive (resp. asymptotically additive) potentials $\Phi_{i}=\left\{\log \phi_{n, i}\right\}_{n=1}^{\infty}$, $i=1, \ldots, k$, and $\mathbf{a}=\left(a_{1}, \ldots, a_{k}\right)$, we define

$$
E_{\boldsymbol{\Phi}}(\mathbf{a})=\left\{x \in X: \lim _{n \rightarrow \infty} \frac{\log \phi_{n, i}(x)}{n}=a_{i} \text { for } 1 \leq i \leq k\right\} .
$$

We indeed obtain the high dimensional versions of Theorems 1.2 1.4 regarding the properties about $h_{\mathrm{top}}\left(T, E_{\boldsymbol{\Phi}}(\mathbf{a})\right)$ and the corresponding variational relations (see Theorems 4.2, 4.8, 
5.2). For instance, when $(X, T)$ is a saturated TDS such that the entropy map is upper-semi continuous, then for any asymptotically additive potentials $\Phi_{i}(i=1, \ldots, k)$, we have

$$
E_{\boldsymbol{\Phi}}(\mathbf{a}) \neq \emptyset \Longleftrightarrow \mathbf{a} \in A:=\left\{\boldsymbol{\Phi}_{*}(\mu): \mu \in \mathcal{M}(X, T)\right\}
$$

and

$$
\begin{aligned}
h_{\mathrm{top}}\left(T, E_{\boldsymbol{\Phi}}(\mathbf{a})\right) & =\sup \left\{h_{\mu}(T): \mu \in \mathcal{M}(X, T) \text { with } \boldsymbol{\Phi}_{*}(\mu)=\mathbf{a}\right\} \\
& =\inf \left\{P_{\boldsymbol{\Phi}}(\mathbf{q})-\mathbf{a} \cdot \mathbf{q}: \mathbf{q} \in \mathbb{R}^{k}\right\}, \quad \forall \mathbf{a} \in A .
\end{aligned}
$$

where $\boldsymbol{\Phi}_{*}(\mu):=\left(\left(\Phi_{1}\right)_{*}(\mu), \cdots,\left(\Phi_{k}\right)_{*}(\mu)\right), P_{\mathbf{\Phi}}(\mathbf{q})=P\left(T, \sum_{i=1}^{k} q_{i} \Phi_{i}\right)$ for $\mathbf{q}=\left(q_{1}, \ldots, q_{k}\right)$, and $\mathbf{a} \cdot \mathbf{q}$ denotes the inner product of $\mathbf{a}$ and $\mathbf{q}$ (see Theorem 5.2 (iii)-(iv)).

As an application of the above result, we can improve a result of Barreira and Gelfert in [6] on Lyapunov exponents on nonconformal repellers. To see it, let $\Lambda$ be a repeller of a $C^{1}$ local diffeomorphism $f: \mathbb{R}^{2} \rightarrow \mathbb{R}^{2}$, such that $f$ satisfies a cone condition on $\Lambda$ (see [6] for the definition). Let $\Phi_{i}=\left\{\log \phi_{n, 1}\right\}_{n=1}^{\infty}(i=1,2)$ are two potentials given by

$$
\phi_{n, i}(x)=\sigma_{i}\left(d_{x} f^{n}\right), \quad n \in \mathbb{N}, i=1,2,
$$

where $\sigma_{i}\left(d_{x} f^{n}\right)(i=1,2)$ denote the singular values of the differential of $f^{n}$ at $x$. Both $\Phi_{1}$ and $\Phi_{2}$ are asymptotically additive (see [6, Proposition 4]). Under the additional assumptions that $f$ is $C^{1+\delta}$ and $f$ has bounded distortion, Barreira and Gelfert showed that $h_{\text {top }}\left(T, E_{\boldsymbol{\Phi}}(\mathbf{a})\right)=\inf \left\{P_{\boldsymbol{\Phi}}(\mathbf{q})-\mathbf{a} \cdot \mathbf{q}: \mathbf{q} \in \mathbb{R}^{k}\right\}$ for each gradient a of $P_{\boldsymbol{\Phi}}$ (see [6. Theorem 1]). However according to our result, these two additional assumptions can be removed (although in this case $P_{\boldsymbol{\Phi}}$ may be not differentiable) and the variational relation holds for each $\mathbf{a} \in A:=\left\{\boldsymbol{\Phi}_{*}(\mu): \mu \in \mathcal{M}(X, T)\right\}$ (we remark that $A$ contains the subdifferentials of $P_{\boldsymbol{\Phi}}$; see Theorem $[3.3)$. Below we give some further remarks.

Remark 1.5. (i) In the definition of sub-additive potential $\Phi=\left\{\log \phi_{n}\right\}$, we admit that $\phi_{n}(x)$ takes the value 0 . As an advantage, we can cover the interesting case that $\phi_{n}(x)=\left\|\prod_{i=0}^{n-1} M\left(T^{i} x\right)\right\|$, where $M$ is an arbitrary continuous matrix-valued function.

(ii) There are some natural examples of asymptotically sub-additive (resp. asymptotically additive) potentials which may not be sub-additive, such as the general potential

$$
\Phi=\left\{\log \mu\left(I_{n}(x)\right)\right\}_{n=1}^{\infty},
$$

where $\mu$ is a weak Gibbs measure on a full shift space over finite symbols and $I_{n}(x)$ denotes the $n$-th cylinder about $x$ (cf. [28] and Proposition A.5(iv)). By the way, the quotient space of all asymptotically additive potentials on $X$ under certain equivalence relation is a separable Banach space endowed with some norm (cf. Remark A.6(ii)). These are two main reasons that we setup the theory for asymptotically sub-additive potentials rather than sub-additive potentials.

(iii) For the proofs of Theorems 1.2, 1.4, we first prove their higher dimensional versions by applying convex analysis and the thermodynamic formalism, then derive the onedimensional versions. Although it looks a bit strange and there are relatively simple 
alternative approaches for the one-dimensional versions, however the extension to higher dimensions along those approaches seems difficult.

(iv) Let $\Phi=\left\{\log \phi_{n}\right\}_{n=1}^{\infty}$, where $\phi_{n}$ 's are non-negative continuous functions on $X$ satisfying

$$
\phi_{n+m}(x) \leq C_{n} \phi_{n}(x) \phi_{m}\left(T^{n} x\right), \quad \forall n, m \in \mathbb{N}, x \in X,
$$

where $\left(C_{n}\right)$ is a sequence of positive numbers with $\lim _{n \rightarrow \infty}(1 / n) \log C_{n}=0$. We do not know whether $\Phi$ is asymptotically sub-additive. However, one can manage to prove Lemma A.2 and Theorem 3.1 for this $\Phi$ by an approach similar to [15]. Furthermore, Theorems 1.1 1.3 remain valid for this kind of potentials.

The content of the paper is following. In Section 2, we give some preliminaries about topological entropy and topological pressures, and we also present and derive some results in convex analysis that are needed in the proof of our theorems. In section 3, we introduce the asymptotically sub-additive thermodynamic formalism and we also set up a formula for the subdifferentials of pressure functions. The high dimensional versions of Theorem 1.2 1.3 are formulated and proved in Section 4. In particular, we give a class of sub-additive potentials on full shifts which satisfy part of (1.7) in Section 4. The high dimensional version of Theorem 1.4 is formulated and proved in Section 5. In Section 6, we give some examples about the irregular multifractal behaviors for additive potentials on TDS's for which the entropy maps is not upper semi-continuous. In Appendix $\mathrm{A}$, we give some properties about asymptotically sub-additive (resp. asymptotically additive potentials). In Appendix B, we summarize the main notation and conventions used in this paper.

\section{Preliminaries}

In this section, we first give the definitions and some properties about the topological entropy of non-compact sets and the topological pressure of non-additive potentials, for which the reader is referred to [12, 3, 43, 15] for more details. Then we present some notation and known facts in convex analysis and derive several results which are need in the proofs of our main results.

2.1. Topological entropy. Let $(X, d)$ be a compact metric space and $T: X \rightarrow X$ a continuous transformation. For any $n \in \mathbb{N}$ we define a new metric $d_{n}$ on $X$ by

$$
d_{n}(x, y)=\max \left\{d\left(T^{k}(x), T^{k}(y)\right): k=0, \ldots, n-1\right\},
$$

and for every $\epsilon>0$ we denote by $B_{n}(x, \epsilon)$ the open ball of radius $\epsilon$ in the metric $d_{n}$ around $x$, i.e., $B_{n}(x, \epsilon)=\left\{y \in X: d_{n}(x, y)<\epsilon\right\}$. Let $Z \subset X$ and $\epsilon>0$. We say that an at most countable collection of balls $\Gamma=\left\{B_{n_{i}}\left(x_{i}, \epsilon\right)\right\}_{i}$ covers $Z$ if $Z \subset \bigcup_{i} B_{n_{i}}\left(x_{i}, \epsilon\right)$. For $\Gamma=\left\{B_{n_{i}}\left(x_{i}, \epsilon\right)\right\}_{i}$, put $n(\Gamma)=\min _{i} n_{i}$. Let $s \geq 0$ and define

$$
M(Z, s, N, \epsilon)=\inf \sum_{i} \exp \left(-s n_{i}\right),
$$


where the infinum is taken over all collections $\Gamma=\left\{B_{n_{i}}\left(x_{i}, \epsilon\right)\right\}$ covering $Z$, such that $n(\Gamma) \geq N$. The quantity $M(Z, s, N, \epsilon)$ does not decrease with $N$, hence the following limit exists:

$$
M(Z, s, \epsilon)=\lim _{N \rightarrow \infty} M(Z, s, N, \epsilon)
$$

There exists a critical value of the parameter s, which we will denote by $h_{\mathrm{top}}(T, Z, \epsilon)$, where $M(Z, s, \epsilon)$ jumps from $\infty$ to 0 , i.e.

$$
M(Z, s, \epsilon)= \begin{cases}0, & s>h_{\mathrm{top}}(T, Z, \epsilon) \\ \infty, & s<h_{\mathrm{top}}(T, Z, \epsilon)\end{cases}
$$

It is clear to see that $h_{\text {top }}(T, Z, \epsilon)$ does not decrease with $\epsilon$, and hence the following limit exists,

$$
h_{\mathrm{top}}(T, Z)=\lim _{\epsilon \rightarrow 0} h_{\mathrm{top}}(T, Z, \epsilon)
$$

We call $h_{\text {top }}(T, Z)$ the topological entropy of $T$ restricted to $Z$ or, simply, the topological entropy of $Z$, when there is no confusion about $T$. In particular we write $h_{\text {top }}(T)$ for $h_{\mathrm{top}}(T, X)$. Here we recall some of the basic properties about the topological entropy.

Proposition 2.1 ([12, 43]). The topological entropy as defined above satisfies the following:

(1) $h_{\text {top }}\left(T, Z_{1}\right) \leq h_{\text {top }}\left(T, Z_{2}\right)$ for any $Z_{1} \subseteq Z_{2} \subseteq X$.

(2) $h_{\text {top }}(T, Z)=\sup _{i} h_{\text {top }}\left(T, Z_{i}\right)$, where $Z=\bigcup_{i=1}^{\infty} Z_{i} \subseteq X$.

(3) Suppose $\mu$ is an invariant measure and $Z \subseteq X$ is such that $\mu(Z)=1$, then $h_{\mathrm{top}}(T, Z) \geq h_{\mu}(T)$, where $h_{\mu}(T)$ is the measure-theoretic entropy.

2.2. Topological pressure. Let $T: X \rightarrow X$ be a continuous transformation of a compact metric space $(X, d)$. For any $n \in \mathbb{N}$, define the metric $d_{n}$ as in (2.1). For any $\epsilon>0$, a set $E \subseteq X$ is said to be a $(n, \epsilon)$-separated subset of $X$ if $d_{n}(x, y)>\epsilon$ for any two different points $x, y \in E$. Let $\Phi=\left\{\log \phi_{n}(x)\right\}_{n=1}^{\infty}$ be a sequence of functions on $X$ for which $\phi_{n}$ is non-negative for each $n$. We define

$$
P_{n}(T, \Phi, \epsilon)=\sup \left\{\sum_{x \in E} \phi_{n}(x): E \text { is a }(n, \epsilon) \text {-separated subset of } X\right\} \text {. }
$$

It is clear that $P_{n}(T, \Phi, \epsilon)$ is a decreasing function of $\epsilon$. Define

$$
P(T, \Phi, \epsilon)=\limsup _{n \rightarrow \infty} \frac{1}{n} \log P_{n}(T, \Phi, \epsilon)
$$

and $P(T, \Phi)=\lim _{\epsilon \rightarrow 0} P(T, \Phi, \epsilon)$. We call $P(T, \Phi)$ the topological pressure of $\Phi$ with respect to $T$ or, simply, the topological pressure of $\Phi$.

2.3. Subdifferentials of convex functions. We first give some notation and basic facts in convex analysis. For details, one is referred to [30, 45].

By a convex combination of points $\mathbf{x}_{1}, \ldots, \mathbf{x}_{m} \in \mathbb{R}^{k}$ we mean a linear combination $\sum_{i=1}^{m} \lambda_{i} \mathbf{x}_{i}$, where $\lambda_{1}+\cdots+\lambda_{m}=1$ and $\lambda_{1}, \ldots, \lambda_{m} \geq 0$. For any subset $M$ of $\mathbb{R}^{k}$, the convex hull $\operatorname{conv}(M)$ of $M$ is the set of all convex combinations of points from $M$. Carathéodory's 
Theorem says that for any subset $M$ of $\mathbb{R}^{k}$, the convex hull $\operatorname{conv}(M)$ is the set of all convex combinations of $k+1$ points from $M$ (cf. [45, Theorem 17.1]).

Let $C$ be a convex subset of $\mathbb{R}^{k}$. A point $\mathbf{x} \in C$ is called an extreme point of $C$ if $\mathbf{x}=p \mathbf{y}+(1-p) \mathbf{z}$ for some $\mathbf{y}, \mathbf{z} \in C$ and $0<p<1$, then $\mathbf{x}=\mathbf{y}=\mathbf{z}$. The set of extreme points of $C$ is denoted by $\operatorname{ext}(C)$. Minkowski's Theorem says that for any nonempty compact convex subset $C$ of $\mathbb{R}^{k}, C=\operatorname{conv}(\operatorname{ext}(C)$ ) (cf. [30, Theorem 2.3.4] or [45, Corollary 18.5.1]). Hence, according to Carathéodory's Theorem and Minkowski's Theorem, each point in a compact convex set $C \subset \mathbb{R}^{k}$ is a convex combination of $k+1$ points from $\operatorname{ext}(C)$.

A point $\mathbf{x} \in C$ is called exposed point of $C$, if $\{\mathbf{x}\}$ is the intersection of $C$ with some supporting hyperplane of $C$. The set of all exposed points of $C$ will be denoted by $\operatorname{expo}(C)$. Straszewicz' Theorem says for any compact convex set $C$ in $\mathbb{R}^{k}, \operatorname{expo}(C)$ is a dense subset of $\operatorname{ext}(C)$ (cf. [45, Theorem 18.6]).

Let $U$ be an open convex subset of $\mathbb{R}^{k}$ and $f$ be a real continuous convex function on $U$. For $\mathbf{x} \in U, \mathbf{a} \in \mathbb{R}^{k}$ is called a subgradient of $f$ at $\mathbf{x}$, if for any $\mathbf{y} \in U$ one has

$$
f(\mathbf{y})-f(\mathbf{x}) \geq \mathbf{a} \cdot(\mathbf{y}-\mathbf{x})
$$

where the dot denotes the dot product. The set of all subgradients at $\mathbf{x}$ is called the subdifferential of $f$ at $\mathbf{x}$ and is denoted $\partial f(\mathbf{x})$. For $\mathbf{x} \in U$, the subdifferential $\partial f(\mathbf{x})$ is always a nonempty convex compact set (cf. [45, Theorem 23.4]). Write

$$
\partial^{e} f(\mathbf{x})=\operatorname{ext}(\partial f(\mathbf{x})) \text {. }
$$

When $\partial^{e} f(\mathbf{x})=\{\mathbf{a}\}$, we say that $f$ is differentiable at $\mathbf{x}$ and write $f^{\prime}(\mathbf{x})=\mathbf{a}$. It is known that $f$ is differentiable for almost every $\mathbf{x} \in U$ (cf. [30, Theorem 4.2.3]). In the case $k=1$, $\partial f(x)=\left[f^{\prime}(x-), f^{\prime}(x+)\right]$ and $\partial^{e} f(x)=\left\{f^{\prime}(x-), f^{\prime}(x+)\right\}$.

Next we define

$$
\partial f(U)=\bigcup_{\mathbf{x} \in U} \partial f(\mathbf{x}) \quad \text { and } \quad \partial^{e} f(U)=\bigcup_{\mathbf{x} \in U} \partial^{e} f(\mathbf{x}) .
$$

Proposition 2.2. Let $U$ be an open convex subset of $\mathbb{R}^{k}$ and $f$ be a real continuous convex function on $U$. Then for each $\mathbf{x} \in U$ and $\mathbf{a} \in \partial^{e} f(\mathbf{x})$, there exists a sequence $\left(\mathbf{x}_{n}\right) \subset U$ such that $\lim _{n \rightarrow \infty} \mathbf{x}_{n}=\mathbf{x}, f$ is differentiable at each point $\mathbf{x}_{n}$ and $\mathbf{a}=\lim _{n \rightarrow \infty} f^{\prime}\left(\mathbf{x}_{n}\right)$.

Proof. Let $\mathbf{x} \in U$. Since $\operatorname{expo}(\partial f(x))$ is dense in $\partial^{e} f(x)$, we only need to show that the lemma holds when $\mathbf{a} \in \operatorname{expo}(\partial f(\mathbf{x}))$. Fix such an $\mathbf{a}$ and write $\mathbf{a}=\left(a_{1}, \cdots, a_{k}\right)$. Then there exists a non-zero vector $\mathbf{t}=\left(t_{1}, \cdots, t_{k}\right) \in \mathbb{R}^{k}$ such that

$$
\mathbf{t} \cdot \mathbf{b}<\mathbf{t} \cdot \mathbf{a} \quad \text { for any } \mathbf{b} \in \partial f(\mathbf{x}) \backslash\{\mathbf{a}\}
$$


Since $f$ is differentiable almost every on $U$, there exists a sequence $\left(\mathbf{x}_{n}\right) \in U$ such that $\lim _{n \rightarrow \infty} \mathbf{x}_{n}=\mathbf{x}, f$ is differentiable at each $\mathbf{x}_{n}$ and

$$
\left|\mathbf{x}_{n}-(\mathbf{x}+\mathbf{t} / n)\right|<n^{-2} \text { for all } n \in \mathbb{N} .
$$

Write $\mathbf{a}_{n}=f^{\prime}\left(\mathbf{x}_{n}\right)$. Note that the sequence $\left(\mathbf{a}_{n}\right)$ is bounded because of the boundedness of $\left(x_{n}\right)$. Hence by taking a subsequence if it is necessary, we can assume that $\lim _{n \rightarrow \infty} \mathbf{a}_{n}=\mathbf{a}^{\prime}$ for some $\mathbf{a}^{\prime} \in \mathbb{R}^{k}$. In the following we show that $\mathbf{a}^{\prime}=\mathbf{a}$.

Since $\mathbf{a}_{n}=f^{\prime}\left(\mathbf{x}_{n}\right)$, one has

$$
f(\mathbf{z})-f\left(\mathbf{x}_{n}\right) \geq \mathbf{a}_{n} \cdot\left(\mathbf{z}-\mathbf{x}_{n}\right) \quad \text { for any } \mathbf{z} \in U .
$$

Letting $n \rightarrow \infty$ yields $f(\mathbf{z})-f(\mathbf{x}) \geq \mathbf{a}^{\prime} \cdot(\mathbf{z}-\mathbf{x})$ for any $\mathbf{z} \in U$, which implies $\mathbf{a}^{\prime} \in \partial f(\mathbf{x})$. Meanwhile for each $n \in \mathbb{N}$,

$$
f(\mathbf{x})-f\left(\mathbf{x}_{n}\right) \geq \mathbf{a}_{n} \cdot\left(\mathbf{x}-\mathbf{x}_{n}\right) \quad \text { and } \quad f\left(\mathbf{x}_{n}\right)-f(\mathbf{x}) \geq \mathbf{a} \cdot\left(\mathbf{x}_{n}-\mathbf{x}\right) .
$$

Hence $\mathbf{a}_{n} \cdot\left(\mathbf{x}_{n}-\mathbf{x}\right) \geq f\left(\mathbf{x}_{n}\right)-f(\mathbf{x}) \geq \mathbf{a} \cdot\left(\mathbf{x}_{n}-\mathbf{x}\right)$. That is,

$$
\mathbf{a}_{n} \cdot\left(\mathbf{t}+n \mathbf{w}_{n}\right) \geq \mathbf{a} \cdot\left(\mathbf{t}+n \mathbf{w}_{n}\right),
$$

where $\mathbf{w}_{n}:=\mathbf{x}_{n}-(\mathbf{x}+\mathbf{t} / n)$. Taking $n \rightarrow \infty$ and noting that $\lim _{n \rightarrow \infty} n\left|\mathbf{w}_{n}\right|=0$ (by (2.4) $)$, we have

$$
\mathbf{a}^{\prime} \cdot \mathbf{t} \geq \mathbf{a} \cdot \mathbf{t}
$$

Combining it with (2.3) and the fact $\mathbf{a}^{\prime} \in \partial f(\mathbf{x})$, one has $\mathbf{a}^{\prime}=\mathbf{a}$. This finishes the proof of the proposition.

Proposition 2.3. Let $Y$ be a compact convex subset of a topological vector space which satisfies the first axiom of countability (i.e., there is a countable base at each point) and $U \subseteq \mathbb{R}^{k}$ a non-empty open convex set. Suppose $f: U \times Y \rightarrow \mathbb{R} \cup\{-\infty\}$ is a map satisfying the following conditions:

(i) $f(\mathbf{q}, y)$ is convex in $\mathbf{q}$;

(ii) $f(\mathbf{q}, y)$ is affine in $y$;

(iii) $f$ is upper semi-continuous over $U \times Y$;

(iv) $g(\mathbf{q}):=\sup _{y \in Y} f(\mathbf{q}, y)>-\infty$ for any $\mathbf{q} \in U$.

For each $\mathbf{q} \in U$, denote $\mathcal{I}(\mathbf{q}):=\{y \in Y: f(\mathbf{q}, y)=g(\mathbf{q})\}$. Then

$$
\partial g(\mathbf{q})=\bigcup_{y \in \mathcal{I}(\mathbf{q})} \partial f(\mathbf{q}, y)
$$

where $\partial f(\mathbf{q}, y)$ denotes the subdifferential of $f(\cdot, y)$ at $\mathbf{q}$.

Proof. By (i)-(iv), $g$ is a real convex function over $U$, and $\mathcal{I}(\mathbf{q})$ is a non-empty compact convex subset of $Y$ for each $\mathbf{q} \in U$. For convenience, denote by $R(\mathbf{q})$ the righthand side of $(2.5)$. A direct check shows that $R(\mathbf{q})$ is a non-empty convex subset of $\mathbb{R}^{k}$ for each $\mathbf{q} \in U$. We further show that for each $\mathbf{q} \in U$, 
(c1) $R(\mathbf{q})$ is compact;

(c2) For each $\delta>0$, there exists $\gamma>0$ such that

$$
R(\mathbf{t}) \subseteq B_{\delta}(R(\mathbf{q})) \quad \text { whenever } \mathbf{t} \in U,|\mathbf{t}-\mathbf{q}|<\gamma,
$$

where $B_{\delta}(R(\mathbf{q})):=\left\{\mathbf{b} \in \mathbb{R}^{k}: d(\mathbf{b}, R(\mathbf{q})) \leq \delta\right\}$ is the closed $\delta$-neighborhood of $R(\mathbf{q})$ in $\mathbb{R}^{k}$.

To show (c1), let $\left(\mathbf{a}_{n}\right)$ be a sequence in $R(\mathbf{q})$. Take $y_{n} \in \mathcal{I}(\mathbf{q})$ so that $\mathbf{a}_{n} \in \partial f\left(\mathbf{q}, y_{n}\right)$. Then

$$
f\left(\mathbf{t}, y_{n}\right)-g(\mathbf{q})=f\left(\mathbf{t}, y_{n}\right)-f\left(\mathbf{q}, y_{n}\right) \geq \mathbf{a}_{n} \cdot(\mathbf{t}-\mathbf{q})
$$

for each $\mathbf{t} \in U$. Hence the sequence $\left(\mathbf{a}_{n}\right)$ should be bounded (otherwise, there exists $\mathbf{t} \in U$ such that $\mathbf{a}_{n} \cdot(\mathbf{t}-\mathbf{q})$ is unbounded from above, however $f\left(\mathbf{t}, y_{n}\right)-f\left(\mathbf{q}, y_{n}\right)=f\left(\mathbf{t}, y_{n}\right)-g(\mathbf{q}) \leq$ $g(\mathbf{t})-g(\mathbf{q}))$. Taking a subsequence if necessary, we assume that $y_{n} \rightarrow y$ and $\mathbf{a}_{n} \rightarrow \mathbf{a}$ for some $y \in \mathcal{I}(\mathbf{q})$ and $\mathbf{a} \in \mathbb{R}^{k}$. Since $f(\mathbf{t}, \cdot)$ is upper semi-continuous, by (2.6) we have $f(\mathbf{t}, y)-f(\mathbf{q}, y)=f(\mathbf{t}, y)-g(\mathbf{q}) \geq \mathbf{a} \cdot(\mathbf{t}-\mathbf{q})$ for each $\mathbf{t} \in U$. This shows $\mathbf{a} \in R(\mathbf{q})$ and hence $R(\mathbf{q})$ is compact. To show (c2), we use contradiction. Assume that (c2) does not hold. Then there exist $\delta>0$ and a sequence $\left(\mathbf{t}_{n}\right)$ in $U$ with $\lim _{n \rightarrow \infty} \mathbf{t}_{n}=\mathbf{q}$ such that there exists $\mathbf{a}_{n} \in R\left(\mathbf{t}_{n}\right)$ satisfying $d\left(\mathbf{a}_{n}, R(\mathbf{q})\right)>\delta$ for each $n$. Take $y_{n} \in \mathcal{I}\left(\mathbf{t}_{n}\right)$ so that $\mathbf{a}_{n} \in \partial f\left(\mathbf{t}_{n}, y_{n}\right)$. Then we have

$$
f\left(\mathbf{t}, y_{n}\right)-g\left(\mathbf{t}_{n}\right)=f\left(\mathbf{t}, y_{n}\right)-f\left(\mathbf{t}_{n}, y_{n}\right) \geq \mathbf{a}_{n} \cdot\left(\mathbf{t}-\mathbf{t}_{n}\right)
$$

for each $\mathbf{t} \in U$. Similarly we can show that $\mathbf{a}_{n}$ is bounded. Hence by taking a subsequence if necessary, we can assume that $y_{n} \rightarrow y$ and $\mathbf{a}_{n} \rightarrow \mathbf{a}$ for some $y \in Y$ and $\mathbf{a} \in \mathbb{R}^{k}$. By the upper semi-continuity of $f$ and the continuity of $g$, we have

$$
f(\mathbf{q}, y) \geq \limsup _{n \rightarrow \infty} f\left(\mathbf{t}_{n}, y_{n}\right)=\limsup _{n \rightarrow \infty} g\left(\mathbf{t}_{n}\right)=g(\mathbf{q}),
$$

which implies $y \in \mathcal{I}(\mathbf{q})$. Hence taking $n \rightarrow \infty$ in (2.7) yields

$$
f(\mathbf{t}, y)-f(\mathbf{q}, y)=f(\mathbf{t}, y)-g(\mathbf{q}) \geq \limsup _{n \rightarrow \infty}\left(f\left(\mathbf{t}, y_{n}\right)-g\left(\mathbf{t}_{n}\right)\right) \geq \mathbf{a} \cdot(\mathbf{t}-\mathbf{q})
$$

for each $\mathbf{t} \in U$. Hence $\mathbf{a} \in \partial f(\mathbf{q}, y)$. Thus $\mathbf{a} \in R(\mathbf{q})$, which contradicts the assumption that $d\left(\mathbf{a}_{n}, R(\mathbf{q})\right)>\delta$ for all $n$. This finishes the proof of (c2).

Now we are ready to show (2.5), i.e., $\partial g(\mathbf{q})=R(\mathbf{q})$. For each $\mathbf{a} \in R(\mathbf{q})$, there exists $y \in \mathcal{I}(\mathbf{q})$ so that $\mathbf{a} \in \partial f(\mathbf{q}, y)$. Hence for each $\mathbf{t} \in U$,

$$
g(\mathbf{t})-g(\mathbf{q}) \geq f(\mathbf{t}, y)-g(\mathbf{q})=f(\mathbf{t}, y)-f(\mathbf{q}, y) \geq \mathbf{a} \cdot(\mathbf{t}-\mathbf{q}) .
$$

This implies $\mathbf{a} \in \partial g(\mathbf{q})$ and thus $\partial g(\mathbf{q}) \supseteq R(\mathbf{q})$.

In the end, we show that $\partial g(\mathbf{q}) \subseteq R(\mathbf{q})$ by contradiction. Assume that $\mathbf{a} \in \partial g(\mathbf{q}) \backslash R(\mathbf{q})$. Since $R(\mathbf{t}) \subseteq \partial g(\mathbf{t})$, we have

$$
g(\mathbf{t})-g(\mathbf{q}) \leq \mathbf{b} \cdot(\mathbf{t}-\mathbf{q}), \quad \forall \mathbf{t} \in U, \mathbf{b} \in R(\mathbf{t}) .
$$


Note that $\mathbf{a} \in \partial g(\mathbf{q})$. We have $g(\mathbf{t})-g(\mathbf{q}) \geq \mathbf{a} \cdot(\mathbf{t}-\mathbf{q})$ for all $\mathbf{t} \in U$. This combining (2.9) yields

$$
\mathbf{a} \cdot(\mathbf{t}-\mathbf{q}) \leq \mathbf{b} \cdot(\mathbf{t}-\mathbf{q}), \quad \forall \mathbf{t} \in U, \mathbf{b} \in R(\mathbf{t}) .
$$

Since $\mathbf{a} \notin R(\mathbf{q})$ and $R(\mathbf{q})$ is compact, there exists $\delta>0$ so that $\mathbf{a} \notin B_{\delta}(R(\mathbf{q}))$. Notice that $B_{\delta}(R(\mathbf{q}))$ is compact convex (since so is $R(\mathbf{q})$ ), there exists a vector $\mathbf{e} \in \mathbb{R}^{k}$ such that $|\mathbf{e}|=1$ and $\mathbf{a} \cdot \mathbf{e}>\mathbf{b} \cdot \mathbf{e}$ for any $\mathbf{b} \in B_{\delta}(R(\mathbf{q}))$. By (c2), there exists $\gamma>0$ such that $R(\mathbf{t}) \subseteq B_{\delta}(R(\mathbf{q}))$ whenever $|\mathbf{t}-\mathbf{q}| \leq \gamma$. Take a small $0<\tilde{\gamma}<\gamma$ such that $\mathbf{t}_{0}:=\mathbf{q}+(\tilde{\gamma} / 2) \mathbf{e} \in U$. Then $\mathbf{a} \cdot\left(\mathbf{t}_{0}-\mathbf{q}\right)>\mathbf{b} \cdot\left(\mathbf{t}_{0}-\mathbf{q}\right)$ for any $\mathbf{b} \in R\left(\mathbf{t}_{0}\right)$, which contradicts (2.10). This proves $\partial g(\mathbf{q}) \subseteq R(\mathbf{q})$.

2.4. Conjugates of convex functions. Let $f: \mathbb{R}^{k} \rightarrow \mathbb{R} \cup\{+\infty\}$ be convex and not identically equal to $+\infty$. Then the function $f^{*}: \mathbb{R}^{k} \rightarrow \mathbb{R} \cup\{+\infty\}$ defined by

$$
\mathbf{s} \mapsto f^{*}(\mathbf{s}):=\sup \left\{\mathbf{s} \cdot \mathbf{x}-f(\mathbf{x}): x \in \mathbb{R}^{k}\right\}
$$

is called the conjugate function of $f$ or Legendre transform of $f$. It is known that $f^{*}$ is also convex and not identically equal to $+\infty$ (cf. [30, p. 211]). Let $f^{* *}$ denote the conjugate of $f^{*}$. The following result is well known (cf. [45, Theorem 12.2]).

Theorem 2.4. Let $f: \mathbb{R}^{k} \rightarrow \mathbb{R} \cup\{+\infty\}$ be convex and not identically equal to $+\infty$. Let $\mathbf{x} \in \mathbb{R}^{k}$. Assume that $f$ is lower semi-continuous at $\mathbf{x}$, i.e., $\liminf _{\mathbf{y} \rightarrow \mathbf{x}} f(\mathbf{y}) \geq f(\mathbf{x})$. Then $f^{* *}(\mathbf{x})=f(\mathbf{x})$.

As an application, we have

Corollary 2.5. Let $A$ be a non-empty convex set in $\mathbb{R}^{k}$ and $g: A \rightarrow \mathbb{R}$ be a concave function. Set

$$
W(\mathbf{x})=\sup \{g(\mathbf{a})+\mathbf{a} \cdot \mathbf{x}: \mathbf{a} \in A\}, \quad \mathbf{x} \in \mathbb{R}^{k}
$$

and

Then we have

$$
G(\mathbf{a})=\inf \left\{W(\mathbf{x})-\mathbf{a} \cdot \mathbf{x}: \mathbf{x} \in \mathbb{R}^{k}\right\}, \quad \mathbf{a} \in \mathbb{R}^{k}
$$

(i) $G(\mathbf{a})=g(\mathbf{a})$ for $\mathbf{a} \in \operatorname{ri}(A)$, where $\operatorname{ri}(A)$ denotes the relative interior of $A$.

(ii) Assume in addition that $A$ is closed. If $g$ is upper semi-continuous at $\mathbf{a} \in A$, then $G(\mathbf{a})=g(\mathbf{a})$.

Proof. Let $f: \mathbb{R}^{k} \rightarrow \mathbb{R} \cup\{+\infty\}$ be the function which agrees with $-g$ on $A$ but is $+\infty$ everywhere else. Then $f$ is convex and has $A$ as its efficient domain, i.e., $A=\{\mathbf{x}: f(\mathbf{x})<$ $+\infty$ \}. By the definition of $W$ and $G$, we have $W=f^{*}$ and $G=-f^{* *}$. However, $f$ is lower semi-continuous on $\operatorname{ri}(A)$ (see, e.g., [45, Theorem 7.4]). Hence by Theorem 2.4, we have $f^{* *}(\mathbf{a})=f(\mathbf{a})$ for $\mathbf{a} \in \operatorname{ri}(A)$, and thus $G(\mathbf{a})=g(\mathbf{a})$ for $\mathbf{a} \in \operatorname{ri}(A)$. This proves (i). To show (ii), assume that $A$ is closed. Let $\mathbf{a} \in A$ so that $g$ is upper semi-continuous at $\mathbf{a}$. Then it is direct to check that $f$ is lower semi-continuous at a. By Theorem 2.4, we have $f^{* *}(\mathbf{a})=f(\mathbf{a})$ and hence $G(\mathbf{a})=g(\mathbf{a})$. This finishes the proof of (ii). 


\section{The THERMODYNAMIC FORMALISM AND SUBDIFFERENTIALS OF PRESSURE FUNCTIONS}

In this section, we firstly introduce a variational principle of topological pressures which plays a key role in the proofs of our main theorems. Then we set up a formula for the subdifferentials of pressure functions.

Let $(X, T)$ be a TDS and let $\Phi=\left\{\log \phi_{n}\right\}_{n=1}^{\infty}$ be an asymptotically sub-additive potential on a $\operatorname{TDS}(X, T)$. Let $\lambda_{\Phi}, \Phi_{*}$ and $\bar{\beta}(\Phi)$ be defined as in (1.2), (1.3) and (1.8). Some basic properties of $\lambda_{\Phi}, \Phi_{*}$ and $\bar{\beta}(\Phi)$ are given in Appendix $\mathrm{A}$. The following variational principle plays a key role in our analysis.

Theorem $3.1([15])$. The topological pressure $P(T, \Phi)$ of $\Phi$ satisfies the following variational principle:

$$
P(T, \Phi)=\left\{\begin{array}{l}
-\infty, \quad \text { if } \Phi_{*}(\mu)=-\infty \text { for all } \mu \in \mathcal{M}(X, T), \\
\sup \left\{h_{\mu}(T)+\Phi_{*}(\mu): \mu \in \mathcal{M}(X, T), \Phi_{*}(\mu) \neq-\infty\right\}, \text { otherwise. }
\end{array}\right.
$$

In particular if $h_{\mathrm{top}}(T)<\infty$, then $P(T, \Phi)=\sup \left\{h_{\mu}(T)+\Phi_{*}(\mu): \mu \in \mathcal{M}(X, T)\right\}$.

The above theorem is only proved in [15, Theorem 1.1] for sub-additive potentials. However the proof given there works well for asymptotically sub-additive potentials, in which we only need to replace Lemma 2.3 in [15] by Lemma A.2 given in Appendix. We remark that the variational principle for sub-additive potentials has been studied in [18, 3, 25, 31, 35, 4, 40] under additional assumptions on the corresponding sub-additive potential and TDS.

In the remain part of this section, we present and prove a formula for the subdifferentials of pressure functions. We first give some notation.

Let $k \in \mathbb{N}$. For each $i=1, \ldots, k$, let $\Phi_{i}=\left\{\log \phi_{n, i}\right\}_{n=1}^{\infty}$ be an asymptotically subadditive potential on $(X, T)$. Write $\mathbb{R}_{+}^{k}=\left\{\left(x_{1}, x_{2}, \ldots, x_{k}\right): x_{i}>0, i=1,2, \cdots, k\right\}$ and $\boldsymbol{\Phi}=\left(\Phi_{1}, \Phi_{2}, \ldots, \Phi_{k}\right)$. For $\mu \in \mathcal{M}(X, T)$, write

$$
\mathbf{\Phi}_{*}(\mu)=\left(\left(\Phi_{1}\right)_{*}(\mu), \cdots,\left(\Phi_{k}\right)_{*}(\mu)\right) .
$$

For $\mathbf{q}=\left(q_{1}, \cdots, q_{k}\right) \in \mathbb{R}_{+}^{k}$, let $\mathbf{q} \cdot \mathbf{\Phi}=\sum_{i=1}^{k} q_{i} \Phi_{i}$ denote the asymptotically sub-additive potential $\left\{\sum_{i=1}^{k} q_{i} \log \phi_{n, i}\right\}_{n=1}^{\infty}$ and write

$$
P_{\boldsymbol{\Phi}}(\mathbf{q})=P(T, \mathbf{q} \cdot \mathbf{\Phi}) .
$$

We call $P_{\boldsymbol{\Phi}}$ the pressure function of $\boldsymbol{\Phi}$.

Let $\bar{\beta}(\boldsymbol{\Phi})=\bar{\beta}\left(\sum_{i=1}^{k} \Phi_{i}\right)$. Then by Theorem 3.1, if $\bar{\beta}(\boldsymbol{\Phi})=-\infty$ then $P_{\boldsymbol{\Phi}}(\mathbf{q})=-\infty$ for any $\mathbf{q} \in \mathbb{R}_{+}^{k}$. If $\bar{\beta}(\boldsymbol{\Phi})>-\infty$, then $\bar{\beta}\left(\Phi_{1}\right)>-\infty, \ldots, \bar{\beta}\left(\Phi_{k}\right)>-\infty$.

Proposition 3.2. Assume that $h_{\mathrm{top}}(T)<\infty$ and $\bar{\beta}(\boldsymbol{\Phi})>-\infty$. Then $P_{\boldsymbol{\Phi}}$ is a real continuous convex function on $\mathbb{R}_{+}^{k}$ and

$$
\partial P_{\boldsymbol{\Phi}}\left(\mathbb{R}_{+}^{k}\right) \subseteq\left(-\infty, \bar{\beta}\left(\Phi_{1}\right)\right] \times\left(-\infty, \bar{\beta}\left(\Phi_{2}\right)\right] \times \cdots \times\left(-\infty, \bar{\beta}\left(\Phi_{k}\right)\right],
$$

where $\partial P_{\boldsymbol{\Phi}}\left(\mathbb{R}_{+}^{k}\right)$ is defined as in (2.2). 
Proof. By Lemma A.3 (4), $P_{\boldsymbol{\Phi}}(\mathbf{q}) \in \mathbb{R}$ for $\mathbf{q} \in \mathbb{R}_{+}^{k}$. The convexity of $P_{\boldsymbol{\Phi}}$ over $\mathbb{R}_{+}^{k}$ just comes from Theorem 3.1, using the affine property of the maps $\mu \rightarrow h_{\mu}(T)$ and $\mu \rightarrow\left(\Phi_{i}\right)_{*}(\mu)$. Since $P_{\boldsymbol{\Phi}}$ is also locally bounded on $\mathbb{R}_{+}^{k}, P_{\boldsymbol{\Phi}}$ is continuous on $\mathbb{R}_{+}^{k}$.

Fix $\mathbf{q}=\left(q_{1}, \cdots, q_{k}\right) \in \mathbb{R}_{+}^{k}$. Define $\mathbf{q}_{\lambda}=\left(q_{1}+\lambda, q_{2}, \cdots, q_{k}\right)$ for $\lambda>0$. Let $\mathbf{a}=$ $\left(a_{1}, \cdots, a_{k}\right) \in \partial P_{\boldsymbol{\Phi}}$. Then

$$
h_{\mathrm{top}}(T)+\lambda \bar{\beta}\left(\Phi_{1}\right)+\sum_{i=1}^{k} q_{i} \bar{\beta}\left(\Phi_{i}\right) \geq P_{\boldsymbol{\Phi}}\left(\mathbf{q}_{\lambda}\right) \geq P_{\boldsymbol{\Phi}}(\mathbf{q})+\left(\mathbf{q}_{\lambda}-\mathbf{q}\right) \cdot \mathbf{a}=P_{\mathbf{\Phi}}(\mathbf{q})+\lambda a_{1} .
$$

Letting $\lambda \rightarrow \infty$ one gets $\bar{\beta}\left(\Phi_{1}\right) \geq \alpha_{1}$. Similarly, we have $\alpha_{i} \leq \bar{\beta}\left(\Phi_{i}\right)$ for $i=2, \cdots, k$.

For $\mathbf{q} \in \mathbb{R}_{+}^{k}$, let $\mathcal{I}(\boldsymbol{\Phi}, \mathbf{q})$ denote the collection of invariant measures $\mu$ such that

$$
h_{\mu}(T)+\mathbf{q} \cdot \boldsymbol{\Phi}_{*}(\mu)=P(T, \mathbf{q} \cdot \mathbf{\Phi}) .
$$

If $\mathcal{I}(\mathbf{\Phi}, \mathbf{q}) \neq \emptyset$, then each element $\mathcal{I}(\mathbf{\Phi}, \mathbf{q})$ is called an equilibrium state for $\mathbf{q} \cdot \mathbf{\Phi}$.

In the following theorem, we set up a formula for the subdifferentials of $P_{\boldsymbol{\Phi}}$, which extends Ruelle's derivative formula for the pressures of additive potentials (cf. [46, exercise 5, p. 99], [41, lemma 4] and [33, theorem 4.3.5]).

Theorem 3.3. Assume that $h_{\mathrm{top}}(T)<\infty, \bar{\beta}(\mathbf{\Phi})>-\infty$, and that the entropy map $\mu \mapsto$ $h_{\mu}(T)$ is upper semi-continuous. Then

(i) For any $\mathbf{q} \in \mathbb{R}_{+}^{k}, \mathcal{I}(\mathbf{\Phi}, \mathbf{q})$ is a non-empty compact convex subset of $\mathcal{M}(X, T)$, and every extreme point of $\mathcal{I}(\mathbf{\Phi}, \mathbf{q})$ is an ergodic measure (i.e., an extreme point of $\mathcal{M}(X, T))$. Furthermore

$$
\partial P_{\boldsymbol{\Phi}}(\mathbf{q})=\left\{\boldsymbol{\Phi}_{*}(\mu): \mu \in \mathcal{I}(\mathbf{\Phi}, \mathbf{q})\right\} .
$$

(ii) Assume in addition that $\Phi_{i}(i=1, \ldots, k)$ are all asymptotically additive. Then the above results hold for all $\mathbf{q} \in \mathbb{R}^{k}$.

Proof. To prove (i), let $\mathbf{q} \in \mathbb{R}_{+}^{k}$. Then $\mathbf{q} \cdot \mathbf{\Phi}$ is an asymptotically sub-additive potential. We first show that $\mathcal{I}(\boldsymbol{\Phi}, \mathbf{q}) \neq \emptyset$. By Theorem 3.1, there exists a sequence $\left\{\mu_{n}\right\} \subset \mathcal{M}(X, T)$ such that $P_{\mathbf{\Phi}}(\mathbf{q})=\lim _{n \rightarrow \infty} h_{\mu_{n}}(T)+\mathbf{q} \cdot \boldsymbol{\Phi}_{*}\left(\mu_{n}\right)$. Let $\mu$ be a limit point of $\left\{\mu_{n}\right\}$. Then by the upper semi-continuity of $h_{(\cdot)}(T)$ and $\left(\Phi_{i}\right)_{*}(\cdot)$, we have $P_{\mathbf{\Phi}}(\mathbf{q}) \leq h_{\mu}(T)+\mathbf{q} \cdot \mathbf{\Phi}_{*}(\mu)$. Applying Theorem 3.1 again we obtain $P_{\boldsymbol{\Phi}}(\mathbf{q})=h_{\mu}(T)+\mathbf{q} \cdot \boldsymbol{\Phi}_{*}(\mu)$, i.e., $\mu \in \mathcal{I}(\mathbf{\Phi}, \mathbf{q})$. Hence $\mathcal{I}(\mathbf{\Phi}, \mathbf{q}) \neq \emptyset$.

An identical argument shows that any limit point of $\mathcal{I}(\boldsymbol{\Phi}, \mathbf{q})$ belongs to $\mathcal{I}(\boldsymbol{\Phi}, \mathbf{q})$ itself. Therefore $\mathcal{I}(\boldsymbol{\Phi}, \mathbf{q})$ is closed and thus compact. Now assume that $\mu$ is an extreme point of $\mathcal{I}(\boldsymbol{\Phi}, \mathbf{q})$. We claim that $\mu$ is ergodic, i.e., $\mu$ is also an extreme point of $\mathcal{M}(X, T)$. To see it, assume $\mu=p \mu_{1}+(1-p) \mu_{2}$ for some $p>0$ and $\mu_{1}, \mu_{2} \in \mathcal{M}(X, T)$. Since $h_{\mu}(T)=$ $p h_{\mu_{1}}(T)+(1-p) h_{\mu_{2}}(T)$ and $\boldsymbol{\Phi}_{*}(\mu)=p \boldsymbol{\Phi}_{*}\left(\mu_{1}\right)+(1-p) \boldsymbol{\Phi}_{*}\left(\mu_{2}\right)$, we have

$$
\begin{aligned}
P_{\boldsymbol{\Phi}}(\mathbf{q}) & =h_{\mu}(T)+\mathbf{q} \cdot \boldsymbol{\Phi}_{*}(\mu) \\
& =p\left(h_{\mu_{1}}(T)+\mathbf{q} \cdot \boldsymbol{\Phi}_{*}\left(\mu_{1}\right)\right)+(1-p)\left(h_{\mu_{2}}(T)+\mathbf{q} \cdot \boldsymbol{\Phi}_{*}\left(\mu_{2}\right)\right) .
\end{aligned}
$$


By Theorem 3.1, we have $\mu_{1}, \mu_{2} \in \mathcal{I}(\mathbf{\Phi}, \mathbf{q})$. Since $\mu$ is an extreme point of $\mathcal{I}(\mathbf{\Phi}, \mathbf{q})$, we have $\mu_{1}=\mu_{2}=\mu$. This shows that $\mu$ is also an extreme point of $\mathcal{M}(X, T)$.

Next we show (3.2). In Proposition 2.3, we take $Y=\mathcal{M}(X, T), U=\mathbb{R}_{+}^{k}$. Define $f: U \times Y \rightarrow \mathbb{R} \cup\{-\infty\}$ by

$$
f(\mathbf{q}, \mu)=\mathbf{q} \cdot \boldsymbol{\Phi}_{*}(\mu)+h_{\mu}(T) .
$$

Then $f$ satisfies the conditions (i)-(iv) in Proposition 2.3. The identity (3.2) just comes from (2.5). This finishes the proof of (i).

Now we turn to the proof of (ii). Assume $\Phi_{i}(i=1, \ldots, k)$ are all asymptotically additive. Let $\mathbf{q} \in \mathbb{R}^{k}$. Then $\mathbf{q} \cdot \boldsymbol{\Phi}$ is also asymptotically additive. Clearly the above proof still work for this case (as a slightly different point, we should take $U=\mathbb{R}^{k}$ for the proof of (3.2)).

\section{MULTIFRACTAL FORMALISM FOR AYMPTOTICALLY SUB-ADDITIVE POTENTIALS}

In the section, we establish the multifractal formalism for asymptotically sub-additive potentials. Let $(X, T)$ be a TDS.

4.1. Proof of Theorem 1.1. Let $\Phi=\left\{\log \phi_{n}\right\}_{n=1}^{\infty}$ be an asymptotically sub-additive potential on $(X, T)$ with $\bar{\beta}(\Phi)>-\infty$. For $x \in X$, we denote by $V(x)$ the set of all limit points in $\mathcal{M}(X)$ of the sequence $\mu_{x, n}=(1 / n) \sum_{j=0}^{n-1} \delta_{T^{j} x}$. This set is a non-empty compact subset of $\mathcal{M}(X, T)$ for each $x$ (cf. [12]). The following result of Bowen plays a key role in the proof of Theorem 1.1 .

Lemma 4.1 (Bowen [12]). For $t \geq 0$, define

$$
R(t)=\left\{x \in X: \exists \mu \in V(x) \text { with } h_{\mu}(T) \leq t\right\} .
$$

Then $h_{\mathrm{top}}(T, R(t)) \leq t$.

Proof of Theorem 1.1. Let $\alpha=\bar{\beta}(\Phi)$. By Lemma A.3(2), there exists $\mu \in \mathcal{E}(X, T)$ so that $\Phi_{*}(\mu)=\alpha$. By Proposition $\mathrm{A} .1(1), \mu\left(E_{\Phi}(\alpha)\right)=1$. Thus $E_{\Phi}(\alpha) \neq \emptyset$. Furthermore by Proposition $2.1(3), h_{\text {top }}\left(T, E_{\Phi}(\alpha)\right) \geq h_{\mu}(T)$. This indeed proves

$$
h_{\mathrm{top}}\left(T, E_{\Phi}(\alpha)\right) \geq \sup \left\{h_{\mu}(T): \mu \in \mathcal{E}(X, T), \Phi_{*}(\mu)=\alpha\right\} .
$$

Now assume $\nu \in \mathcal{M}(X, T)$ so that $\Phi_{*}(\nu)=\alpha$. Let $\nu=\int_{\mathcal{E}(X, T)} \theta d m(\theta)$ be the ergodic decomposition of $\mu$. By Proposition A.1 (3), $\alpha=\Phi_{*}(\nu)=\int_{\mathcal{E}(X, T)} \Phi_{*}(\theta) d m(\theta)$. Since $\alpha \geq \Phi_{*}(\theta)$ for each $\theta \in \mathcal{E}(X, T)$, we have $\alpha=\Phi_{*}(\theta)$ whenever $\theta \in \Omega^{\prime}$, where $\Omega^{\prime}$ is a subset 
of $\mathcal{E}(X, T)$ with $m\left(\Omega^{\prime}\right)=1$. Hence

$$
\begin{aligned}
h_{\nu}(T)+\Phi_{*}(\nu) & =\int_{\mathcal{E}(X, T)}\left(h_{\theta}(T)+\Phi_{*}(\theta)\right) d m(\theta)=\int_{\Omega^{\prime}}\left(h_{\theta}(T)+\Phi_{*}(\theta)\right) d m(\theta) \\
& \leq \int_{\Omega^{\prime}} \sup \left\{h_{\mu}(T): \mu \in \mathcal{E}(X, T), \Phi_{*}(\mu)=\alpha\right\} d m(\theta)+\alpha \\
& =\sup \left\{h_{\mu}(T): \mu \in \mathcal{E}(X, T), \Phi_{*}(\mu)=\alpha\right\}+\alpha .
\end{aligned}
$$

This proves

$$
\sup \left\{h_{\mu}(T): \mu \in \mathcal{M}(X, T), \Phi_{*}(\mu)=\alpha\right\} \leq \sup \left\{h_{\mu}(T): \mu \in \mathcal{E}(X, T), \Phi_{*}(\mu)=\alpha\right\} .
$$

Next we prove that

$$
h_{\mathrm{top}}\left(T, E_{\Phi}(\alpha)\right) \leq \sup \left\{h_{\mu}(T): \mu \in \mathcal{M}(X, T), \Phi_{*}(\mu)=\alpha\right\} .
$$

Denote by $t$ the right-hand side of the above inequality. We may assume that $t<\infty$, otherwise there is nothing remained to prove. Let $x \in E_{\Phi}(\alpha)$ and $\mu \in V(x)$. Then there is $n_{i} \rightarrow \infty$ such that $\mu_{x, n_{i}}=\frac{1}{n_{i}} \sum_{i=0}^{n_{i}-1} \delta_{T^{i} x} \rightarrow \mu$. By Lemma A.2, $\mu \in \mathcal{M}(X, T)$ and $\alpha=$ $\lim _{i \rightarrow \infty} \frac{\log \phi_{n_{i}}(x)}{n_{i}} \leq \Phi_{*}(\mu)$. Moreover $\alpha=\Phi_{*}(\mu)$ by Lemma A.3 $(2)$. Hence $h_{\mu}(T) \leq t$. It follows that

$$
E_{\Phi}(\alpha) \subset R(t):=\left\{x \in X: \exists \mu \in V(x) \text { with } h_{\mu}(T) \leq t\right\} .
$$

By Lemma 4.1, we have $h_{\text {top }}\left(T, E_{\Phi}(\alpha)\right) \leq h_{\text {top }}(T, R(t)) \leq t$. This proves (4.3). Now Theorem 1.1 just follows from (4.1)-(4.3).

4.2. A high dimensional version of Theorem 1.2. In this subsection, we present and prove a high dimension version of Theorem 1.2 .

We first give some notation. Let $k \in \mathbb{N}$. For each $i=1, \ldots, k$, let $\Phi_{i}=\left\{\log \phi_{n, i}\right\}_{n=1}^{\infty}$ be an asymptotically sub-additive potential on $(X, T)$. For $\mathbf{a}=\left(a_{1}, \ldots, a_{k}\right) \in \mathbb{R}^{k}$, let

$$
E_{\boldsymbol{\Phi}}(\mathbf{a})=\left\{x \in X: \lambda_{\Phi_{i}}(x)=a_{i}, i=1,2, \ldots, k\right\} .
$$

For any $\mathbf{b}=\left(b_{1}, \ldots, b_{k}\right) \in \mathbb{R}^{k}$, define

$$
|\mathbf{b}|:=\max \left\{\left|b_{i}\right|: i=1, \ldots, k\right\} .
$$

For $\mathbf{x}=\left(x_{1}, \ldots, x_{k}\right)$ and $\mathbf{y}=\left(y_{1}, \ldots, y_{k}\right) \in \mathbb{R}^{k}$, we write $\mathbf{x} \geq \mathbf{y}$ if $x_{i} \geq y_{i}$ for all $1 \leq i \leq k$. For $A \subseteq \mathbb{R}^{k}$, write

$$
\operatorname{cl}_{+}(A)=\left\{\mathbf{x} \in \mathbb{R}^{k}: \exists\left(\mathbf{y}_{j}\right) \subset A \text { such that } \mathbf{x} \geq \mathbf{y}_{j} \text { and } \lim _{j \rightarrow \infty} \mathbf{y}_{j}=\mathbf{x}\right\} .
$$

For a real valued function $f$ defined on a convex open set $U \subset \mathbb{R}^{k}$, let $\partial f(\mathbf{x}), \partial^{e} f(\mathbf{x})$, $(\mathbf{x} \in U), \partial f(U)$ and $\partial^{e} f(U)$ be defined as in Section 2.3. The following result is a high dimensional version of Theorem 1.2 .

Theorem 4.2. Assume $h_{\mathrm{top}}(T)<\infty$ and $\bar{\beta}(\mathbf{\Phi})>-\infty$. Then $P_{\boldsymbol{\Phi}}$ is a real continuous convex function on $\mathbb{R}_{+}^{k}$. Moreover, 
(i) For any $\mathbf{t} \in \mathbb{R}_{+}^{k}$, if $\mathbf{a} \in \partial^{e} P_{\boldsymbol{\Phi}}(\mathbf{t})$, then

$$
\lim _{\epsilon \rightarrow 0} h_{\text {top }}\left(T, \underset{|\mathbf{b}-\mathbf{a}|<\epsilon}{\bigcup} E_{\boldsymbol{\Phi}}(\mathbf{b})\right)=\inf _{\mathbf{q} \in \mathbb{R}_{+}^{k}}\left\{P_{\boldsymbol{\Phi}}(\mathbf{q})-\mathbf{a} \cdot \mathbf{q}\right\}=P_{\boldsymbol{\Phi}}(\mathbf{t})-\mathbf{a} \cdot \mathbf{t} .
$$

Moreover the first equality is also valid when $\mathbf{a} \in \mathrm{cl}_{+}\left(\partial^{e} P_{\boldsymbol{\Phi}}\left(\mathbb{R}_{+}^{k}\right)\right)$.

(ii) For any $\mathbf{t} \in \mathbb{R}_{+}^{k}$, if $\mathbf{a} \in \partial P_{\mathbf{\Phi}}(\mathbf{t})$, then

$$
\lim _{\epsilon \rightarrow 0} \sup \left\{h_{\mu}(T): \mu \in \mathcal{M}(X, T),\left|\Phi_{*}(\mu)-\mathbf{a}\right|<\epsilon\right\}=\inf _{\mathbf{q} \in \mathbb{R}_{+}^{k}}\left\{P_{\boldsymbol{\Phi}}(\mathbf{q})-\mathbf{a} \cdot \mathbf{q}\right\} .
$$

Furthermore the above equality is valid for $\mathbf{a} \in \mathrm{cl}_{+}\left(\partial P_{\boldsymbol{\Phi}}\left(\mathbb{R}_{+}^{k}\right)\right)$.

(iii) For any $\mathbf{a} \in \partial P_{\boldsymbol{\Phi}}\left(\mathbb{R}_{+}^{k}\right) \cap \operatorname{ri}(A)$,

$$
\inf _{\mathbf{q} \in \mathbb{R}_{+}^{k}}\left\{P_{\mathbf{\Phi}}(\mathbf{q})-\mathbf{a} \cdot \mathbf{q}\right\}=\sup \left\{h_{\mu}(T): \boldsymbol{\Phi}_{*}(\mu)=\mathbf{a}\right\},
$$

where $A:=\left\{\mathbf{a} \in \mathbb{R}^{k}: \mathbf{a}=\mathbf{\Phi}_{*}(\mu)\right.$ for some $\left.\mu \in \mathcal{M}(X, T)\right\}$, and ri denotes the relative interior ( $c f$. [45]).

Remark 4.3. When $\Phi_{i}(i=1, \ldots k)$ are all asymptotically additive, the results in Theorem 4.2 can be extended accordingly. Indeed, one can replace all the terms $\mathbb{R}_{+}^{k}$ in Theorem 4.2 by $\mathbb{R}^{k}$, except the two terms in $\mathrm{cl}_{+}\left(\partial^{e} P_{\boldsymbol{\Phi}}\left(\mathbb{R}_{+}^{k}\right)\right)$ and $\mathrm{cl}_{+}\left(\partial P_{\boldsymbol{\Phi}}\left(\mathbb{R}_{+}^{k}\right)\right)$.

To prove Theorem 4.2, we need some preparations. For any $\mathbf{a}=\left(a_{1}, \cdots, a_{k}\right) \in \mathbb{R}^{k}$ and $\epsilon>0$, define

$$
G(\mathbf{a}, n, \epsilon):=\left\{x \in X:\left|\frac{1}{\ell} \log \phi_{\ell, i}(x)-a_{i}\right|<\epsilon \text { for all } 1 \leq i \leq k \text { and } \ell \geq n\right\} .
$$

We have the following

Lemma 4.4. Assume that $G(\mathbf{a}, n, \epsilon) \neq \emptyset$. Then

(i) For any $\mathbf{q}=\left(q_{1}, \cdots, q_{k}\right) \in \mathbb{R}_{+}^{k}$,

$$
h_{\mathrm{top}}(T, G(\mathbf{a}, n, \epsilon)) \leq P_{\mathbf{\Phi}}(\mathbf{q})-\sum_{i=1}^{k}\left(a_{i}-\epsilon\right) q_{i} .
$$

(ii) Assume furthermore that all $\Phi_{i}(i=1, \ldots, k)$ are asymptotically additive. Then for any $\mathbf{q}=\left(q_{1}, \cdots, q_{k}\right) \in \mathbb{R}^{k}$,

$$
h_{\mathrm{top}}(T, G(\mathbf{a}, n, \epsilon)) \leq P_{\mathbf{\Phi}}(\mathbf{q})-\mathbf{a} \cdot \mathbf{q}+\epsilon \sum_{i=1}^{k}\left|q_{i}\right| .
$$

Proof. We first prove (i). Fix $\mathbf{q}=\left(q_{1}, \ldots, q_{k}\right) \in \mathbb{R}_{+}^{k}$. It suffices to show that for any $s<h_{\text {top }}(T, G(\mathbf{a}, n, \epsilon))$,

$$
P_{\mathbf{\Phi}}(\mathbf{q}) \geq s+\sum_{i=1}^{k}\left(a_{i}-\epsilon\right) q_{i}
$$


Let $s<h_{\text {top }}(T, G(\mathbf{a}, n, \epsilon))$ be given. By definition (cf. Section 2.1), there exists $\gamma>0$ such that $h_{\text {top }}(T, G(\mathbf{a}, n, \epsilon), \gamma)>s$. Therefore (cf. Section 2.1)

$$
\infty=M(G(\mathbf{a}, n, \epsilon), s, \gamma)=\lim _{N \rightarrow \infty} M(G(\mathbf{a}, n, \epsilon), s, N, \gamma)
$$

Hence there exists $N_{0}$ such that

$$
M(G(\mathbf{a}, n, \epsilon), s, N, \gamma) \geq 1, \quad \forall N \geq N_{0} .
$$

Now take $N \geq \max \left\{n, N_{0}\right\}$ and let $F$ be a $(N, \gamma)$-separated subset of $G(\mathbf{a}, n, \epsilon)$ with the maximal cardinality. Then $\bigcup_{x \in F} B_{N}(x, \gamma) \supseteq G(\mathbf{a}, n, \epsilon)$. It follows

$$
\# F \cdot \exp (-s N) \geq M(G(\mathbf{a}, n, \epsilon), s, N, \gamma) \geq 1 \text {. }
$$

Since $\sum_{i=1}^{k} q_{i} \log \phi_{N, i}(x) \geq N\left(\sum_{i=1}^{k}\left(a_{i}-\epsilon\right) q_{i}\right)$ for each $x \in G(\mathbf{a}, n, \epsilon)$, we have

$$
P_{N}(T, \mathbf{q} \cdot \boldsymbol{\Phi}, \gamma) \geq \sum_{x \in F} \exp \left(\sum_{i=1}^{k} q_{i} \log \phi_{N, i}(x)\right) \geq \# F \cdot \exp \left(N\left(\sum_{i=1}^{k}\left(a_{i}-\epsilon\right) q_{i}\right)\right) .
$$

Combining this with (4.7) yields $P_{N}(T, \mathbf{q} \cdot \mathbf{\Phi}, \gamma) \geq \exp \left(N\left(s+\sum_{i=1}^{k}\left(a_{i}-\epsilon\right) q_{i}\right)\right)$. Taking $N \rightarrow \infty$ we obtain $P(T, \mathbf{q} \cdot \mathbf{\Phi}, \gamma) \geq s+\sum_{i=1}^{k}\left(a_{i}-\epsilon\right) q_{i}$. Hence we have

$$
P_{\boldsymbol{\Phi}}(\mathbf{q})=P(T, \mathbf{q} \cdot \mathbf{\Phi}) \geq s+\sum_{i=1}^{k}\left(a_{i}-\epsilon\right) q_{i},
$$

which finishes the proof (i).

The proof of (ii) is almost identical. The only difference part is to use the inequality

$$
\sum_{i=1}^{k} q_{i} \log \phi_{N, i}(x) \geq N\left(\sum_{i=1}^{k}\left(a_{i} q_{i}-\epsilon\left|q_{i}\right|\right)\right.
$$

for each $x \in G(\mathbf{a}, n, \epsilon)$ and $\mathbf{q} \in \mathbb{R}^{k}$.

As a corollary, we have

Corollary 4.5. Let $\mathbf{a}=\left(a_{1}, \ldots, a_{k}\right) \in \mathbb{R}^{k}$ and $\epsilon>0$. Let $E_{\boldsymbol{\Phi}}(\mathbf{a})$ be defined as in (4.4). Then

$$
h_{\text {top }}\left(T, \underset{|\mathbf{b}-\mathbf{a}|<\epsilon}{\bigcup} E_{\boldsymbol{\Phi}}(\mathbf{b})\right) \leq P_{\boldsymbol{\Phi}}(\mathbf{q})-\sum_{i=1}^{k}\left(a_{i}-\epsilon\right) q_{i} \quad \text { for any } \mathbf{q}=\left(q_{1}, \ldots, q_{k}\right) \in \mathbb{R}_{+}^{k},
$$

whenever $\bigcup_{|\mathbf{b}-\mathbf{a}|<\epsilon} E_{\boldsymbol{\Phi}}(\mathbf{b}) \neq \emptyset$. Furthermore if all $\Phi_{i}(i=1, \ldots, k)$ are asymptotically additive, then

$$
h_{\text {top }}\left(T, \underset{|\mathbf{b}-\mathbf{a}|<\epsilon}{\bigcup} E_{\boldsymbol{\Phi}}(\mathbf{b})\right) \leq P_{\boldsymbol{\Phi}}(\mathbf{q})-\mathbf{a} \cdot \mathbf{q}+\epsilon \sum_{i=1}^{k}\left|q_{i}\right|, \quad \forall \mathbf{q}=\left(q_{1}, \ldots, q_{k}\right) \in \mathbb{R}^{k},
$$

whenever $\bigcup_{|\mathbf{b}-\mathbf{a}|<\epsilon} E_{\boldsymbol{\Phi}}(\mathbf{b}) \neq \emptyset$. 
Proof. Observe that $\bigcup_{|\mathbf{b}-\mathbf{a}|<\epsilon} E_{\boldsymbol{\Phi}}(\mathbf{b}) \subseteq \bigcup_{n=1}^{\infty} G(\mathbf{a}, n, \epsilon)$. By Proposition 2.1, we obtain

$$
\begin{aligned}
h_{\mathrm{top}}\left(T, \bigcup_{|\mathbf{b}-\mathbf{a}|<\epsilon} E_{\boldsymbol{\Phi}}(\mathbf{b})\right) & \leq h_{\mathrm{top}}\left(T, \bigcup_{n=1}^{\infty} G(\mathbf{a}, n, \epsilon)\right) \\
& =\sup _{n \geq 1} h_{\mathrm{top}}(T, G(\mathbf{a}, n, \epsilon)) .
\end{aligned}
$$

By Lemma 4.4, we obtain the desired result.

Lemma 4.6. Assume $h_{\mathrm{top}}(T)<\infty$ and $\bar{\beta}(\boldsymbol{\Phi})>-\infty$. Let $\mathbf{q} \in \mathbb{R}_{+}^{k}$. Then for any $\epsilon>0$, there exists $\nu \in \mathcal{E}(X, T)$ such that $h_{\nu}(T)+\mathbf{q} \cdot \mathbf{\Phi}_{*}(\nu) \geq P_{\mathbf{\Phi}}(\mathbf{q})-\epsilon$.

Proof. Let $\epsilon>0$. By Theorem 3.1, there exists $\mu \in \mathcal{M}(X, T)$ such that

$$
h_{\mu}(T)+\mathbf{q} \cdot \boldsymbol{\Phi}_{*}(\mu) \geq P_{\boldsymbol{\Phi}}(\mathbf{q})-\epsilon .
$$

Let $\mu=\int_{\mathcal{E}(X, T)} \theta d m(\theta)$ be the ergodic decomposition of $\mu$. Then by Proposition A.1(3), we have

$$
\int_{\mathcal{E}(X, T)}\left(h_{\theta}(T)+\mathbf{q} \cdot \mathbf{\Phi}_{*}(\theta)\right) d m(\theta)=h_{\mu}(T)+\mathbf{q} \cdot \boldsymbol{\Phi}_{*}(\mu) \geq P_{\mathbf{\Phi}}(\mathbf{q})-\epsilon .
$$

Hence there exists at least one $\nu \in \mathcal{E}(X, T)$ such that $h_{\nu}(T)+\mathbf{q} \cdot \boldsymbol{\Phi}_{*}(\nu) \geq P_{\mathbf{\Phi}}(\mathbf{q})-\epsilon$.

The following result is important in the proof of Theorem 4.2 ,

Lemma 4.7. Assume $h_{\text {top }}(T)<\infty$ and $\bar{\beta}(\mathbf{\Phi})>-\infty$. Let $\mathbf{t} \in \mathbb{R}_{+}^{k}$. Assume $\mathbf{a} \in \partial^{e} P_{\boldsymbol{\Phi}}(\mathbf{t})$. Then for any $\epsilon>0$, there exists $\nu \in \mathcal{E}(X, T)$ such that $\left|\mathbf{\Phi}_{*}(\nu)-\mathbf{a}\right|<\epsilon$ and $\mid h_{\nu}(T)-\left(P_{\mathbf{\Phi}}(\mathbf{t})-\right.$ $\mathbf{a} \cdot \mathbf{t}) \mid<\epsilon$.

Proof. By Proposition [3.2, $P_{\boldsymbol{\Phi}}$ is a real continuous convex function on $\mathbb{R}_{+}^{k}$. Let $\mathbf{t}=$ $\left(t_{1}, \ldots, t_{k}\right) \in \mathbb{R}_{+}^{k}$ and $\epsilon>0$. We first assume that $P_{\boldsymbol{\Phi}}$ is differentiable at $\mathbf{t}$. Let $\mathbf{a}=P_{\boldsymbol{\Phi}}^{\prime}(\mathbf{t})$ and write $\mathbf{a}=\left(a_{1}, \ldots, a_{k}\right)$. Set $\delta=\min \left\{\frac{\epsilon}{3 k}, \frac{\epsilon}{3 k|\mathbf{t}|}\right\}$. Choose $\gamma_{0}>0$ such that

$$
\frac{\left|P_{\boldsymbol{\Phi}}(\mathbf{t}+\mathbf{s})-P_{\boldsymbol{\Phi}}(\mathbf{t})-\mathbf{a} \cdot \mathbf{s}\right|}{|\mathbf{s}|}<\delta \quad \text { for all } \mathbf{s} \in \mathbb{R}^{k} \text { with } 0<|\mathbf{s}| \leq \gamma_{0} .
$$

Pick $\eta$ such that $0<\eta \leq \min \left\{\epsilon / 3, \delta \gamma_{0}\right\}$. By Lemma 4.6, there exists $\nu \in \mathcal{E}(X, T)$ such that

$$
h_{\nu}(T)+\mathbf{t} \cdot \boldsymbol{\Phi}_{*}(\nu) \geq P_{\mathbf{\Phi}}(\mathbf{t})-\eta .
$$

Meanwhile by Theorem 3.1 ,

$$
h_{\nu}(T)+(\mathbf{t}+\mathbf{s}) \cdot \boldsymbol{\Phi}_{*}(\nu) \leq P_{\boldsymbol{\Phi}}(\mathbf{t}+\mathbf{s}) \quad \text { for all } \mathbf{s} \in \mathbb{R}^{k} \text { with } \mathbf{t}+\mathbf{s} \in \mathbb{R}_{+}^{k} .
$$

Combining (4.10) and (4.9) yields

$$
P_{\boldsymbol{\Phi}}(\mathbf{t}+\mathbf{s})-P_{\boldsymbol{\Phi}}(\mathbf{t}) \geq \mathbf{s} \cdot \boldsymbol{\Phi}_{*}(\nu)-\eta \quad \text { for all } \mathbf{s} \in \mathbb{R}^{k} \text { with } \mathbf{t}+\mathbf{s} \in \mathbb{R}_{+}^{k} .
$$


Construct points $\mathbf{s}_{i} \in \mathbb{R}^{k}(i=1, \ldots, k)$ by $\mathbf{s}_{i}=\left(s_{i, 1}, \ldots, s_{i, k}\right)$, where

$$
s_{i, j}= \begin{cases}0 & \text { if } i \neq j \\ \gamma_{0} & \text { if } i=j .\end{cases}
$$

Taking $\mathbf{s}= \pm \mathbf{s}_{i}$ in (4.11) yields

$$
\frac{P_{\boldsymbol{\Phi}}\left(\mathbf{t}+\mathbf{s}_{i}\right)-P_{\boldsymbol{\Phi}}(\mathbf{t})}{\gamma_{0}} \geq\left(\Phi_{i}\right)_{*}(\nu)-\frac{\eta}{\gamma_{0}} \quad \text { and } \quad \frac{P_{\boldsymbol{\Phi}}\left(\mathbf{t}-\mathbf{s}_{i}\right)-P_{\boldsymbol{\Phi}}(\mathbf{t})}{-\gamma_{0}} \leq\left(\Phi_{i}\right)_{*}(\nu)+\frac{\eta}{\gamma_{0}} .
$$

Combining the above two inequalities with (4.8), we have

$$
\left|\left(\Phi_{i}\right)_{*}(\nu)-a_{i}\right| \leq \delta+\frac{\eta}{\gamma_{0}} \leq 2 \delta<\epsilon, \quad i=1, \ldots, k,
$$

which combining with (4.9) and (4.10) yields

$$
h_{\nu}(T) \geq P_{\boldsymbol{\Phi}}(\mathbf{t})-\eta-\sum_{i=1}^{k} t_{i}\left(a_{i}+2 \delta\right) \geq P_{\boldsymbol{\Phi}}(\mathbf{t})-\mathbf{a} \cdot \mathbf{t}-\eta-2 k|\mathbf{t}| \delta>P_{\boldsymbol{\Phi}}(\mathbf{t})-\mathbf{a} \cdot \mathbf{t}-\epsilon .
$$

and

$$
h_{\nu}(T) \leq P_{\boldsymbol{\Phi}}(\mathbf{t})-\mathbf{t} \cdot \boldsymbol{\Phi}_{*}(\nu) \leq P_{\boldsymbol{\Phi}}(\mathbf{t})-\sum_{i=1}^{k} t_{i}\left(a_{i}-2 \delta\right)<P_{\boldsymbol{\Phi}}(\mathbf{t})-\mathbf{a} \cdot \mathbf{t}+\epsilon .
$$

This proves the lemma in the case that $P_{\boldsymbol{\Phi}}$ is differentiable at $\mathbf{t}$.

Now assume that $P_{\boldsymbol{\Phi}}$ is not differentiable at $\mathbf{t}$. Let $\mathbf{a}=\left(a_{1}, \ldots, a_{k}\right) \in \partial^{e} P_{\boldsymbol{\Phi}}(\mathbf{t})$. Since $P_{\boldsymbol{\Phi}}$ is a real continuous convex function on $\mathbb{R}_{+}^{k}$, by Proposition 2.2, there exists a sequence $\left(\mathbf{t}_{n}\right) \subset \mathbb{R}_{+}^{k}$ converging to $\mathbf{t}$ such that $P_{\boldsymbol{\Phi}}^{\prime}\left(\mathbf{t}_{n}\right)$ exists for each $n$ and $\lim _{n \rightarrow \infty} P_{\boldsymbol{\Phi}}^{\prime}\left(\mathbf{t}_{n}\right)=\mathbf{a}$. Choose a large integer $n$ such that

$$
\left|P_{\boldsymbol{\Phi}}^{\prime}\left(\mathbf{t}_{n}\right)-\mathbf{a}\right|<\frac{\epsilon}{2} \quad \text { and } \quad\left|\left(P_{\boldsymbol{\Phi}}\left(\mathbf{t}_{n}\right)-P_{\boldsymbol{\Phi}}^{\prime}\left(\mathbf{t}_{n}\right) \cdot \mathbf{t}_{n}\right)-\left(P_{\boldsymbol{\Phi}}(\mathbf{t})-\mathbf{a} \cdot \mathbf{t}\right)\right|<\frac{\epsilon}{2} .
$$

As proved in the last paragraph, we can choose $\nu \in \mathcal{E}(X, T)$ such that

$$
\left|\boldsymbol{\Phi}_{*}(\nu)-P_{\boldsymbol{\Phi}}^{\prime}\left(\mathbf{t}_{n}\right)\right|<\frac{\epsilon}{2} \quad \text { and } \quad\left|h_{\nu}(T)-\left(P_{\boldsymbol{\Phi}}\left(\mathbf{t}_{n}\right)-P_{\boldsymbol{\Phi}}^{\prime}\left(\mathbf{t}_{n}\right) \cdot \mathbf{t}_{n}\right)\right|<\frac{\epsilon}{2} .
$$

Combining (4.12) with (4.13) yields $\left|\boldsymbol{\Phi}_{*}(\nu)-\mathbf{a}\right|<\epsilon$ and $\left|h_{\nu}(T)-\left(P_{\mathbf{\Phi}}(\mathbf{t})-\mathbf{a} \cdot \mathbf{t}\right)\right|<\epsilon$. This finishes the proof of the lemma.

Proof of Theorem 4.2. By Proposition [3.2, $P_{\boldsymbol{\Phi}}$ is a real continuous convex function on $\mathbb{R}_{+}^{k}$.

We first prove part (i) of the theorem. Let $\mathbf{t}=\left(t_{1}, \ldots, t_{k}\right) \in \mathbb{R}_{+}^{k}$ and $\mathbf{a}=\left(a_{1}, \ldots, a_{k}\right) \in$ $\partial^{e} P_{\boldsymbol{\Phi}}(\mathbf{t})$. Let $\epsilon>0$. Then by Lemma 4.7, there exists $\nu \in \mathcal{E}(X, T)$ such that

$$
\left|\boldsymbol{\Phi}_{*}(\nu)-\mathbf{a}\right|<\epsilon \quad \text { and } \quad\left|h_{\nu}(T)-\left(P_{\mathbf{\Phi}}(\mathbf{t})-\mathbf{a} \cdot \mathbf{t}\right)\right|<\epsilon .
$$

Since $\nu \in \mathcal{E}(X, T)$, by Proposition A.1 $(1), \lambda_{\Phi_{i}}(x)=\left(\Phi_{i}\right)_{*}(\nu)$ for $\nu$-a.e. $x \in X, i=1, \ldots, k$. That is, $\nu\left(E_{\boldsymbol{\Phi}}\left(\boldsymbol{\Phi}_{*}(\nu)\right)\right)=1$. By Proposition [2.1(3), $h_{\text {top }}\left(T, E_{\boldsymbol{\Phi}}\left(\boldsymbol{\Phi}_{*}(\nu)\right)\right) \geq h_{\nu}(T)$. Since 
$\boldsymbol{\Phi}_{*}(\nu)$ and $h_{\nu}(T)$ satisfy (4.14), we have

$$
h_{\text {top }}\left(T, \underset{|\mathbf{b}-\mathbf{a}|<\epsilon}{\bigcup} E_{\boldsymbol{\Phi}}(\mathbf{b})\right) \geq h_{\text {top }}\left(T, E_{\boldsymbol{\Phi}}\left(\boldsymbol{\Phi}_{*}(\nu)\right)\right) \geq h_{\nu}(T) \geq P_{\mathbf{\Phi}}(\mathbf{t})-\mathbf{a} \cdot \mathbf{t}-\epsilon .
$$

On the other hand by Corollary 4.5, we have

$$
h_{\text {top }}\left(T, \bigcup_{|\mathbf{b}-\mathbf{a}|<\epsilon} E_{\boldsymbol{\Phi}}(\mathbf{b})\right) \leq P_{\boldsymbol{\Phi}}(\mathbf{t})-\sum_{i=1}^{k}\left(a_{i}-\epsilon\right) t_{i} .
$$

Combining the above two inequalities and letting $\epsilon \rightarrow 0$, we obtain

$$
\lim _{\epsilon \rightarrow 0} h_{\text {top }}\left(T, \underset{|\mathbf{b}-\mathbf{a}|<\epsilon}{\bigcup} E_{\boldsymbol{\Phi}}(\mathbf{b})\right)=P_{\boldsymbol{\Phi}}(\mathbf{t})-\mathbf{a} \cdot \mathbf{t}=\inf _{\mathbf{q} \in \mathbb{R}_{+}^{k}}\left\{P_{\boldsymbol{\Phi}}(\mathbf{q})-\mathbf{a} \cdot \mathbf{q}\right\} .
$$

Now assume $\mathbf{a} \in \operatorname{cl}_{+}\left(\partial^{e} P_{\boldsymbol{\Phi}}\left(\mathbb{R}^{k}\right)\right)$. Then there exist $\mathbf{t}_{j} \in \mathbb{R}_{+}^{k}$ and $\mathbf{b}_{j} \in \partial^{e} P_{\boldsymbol{\Phi}}\left(\mathbf{t}_{j}\right), j \in \mathbb{N}$ such that $\mathbf{a} \geq \mathbf{b}_{j}$ and $\lim _{j \rightarrow \infty} \mathbf{b}_{j}=\mathbf{a}$. Let $\epsilon>0$. There exists a large $j_{\epsilon}$ such that $\left|\mathbf{a}-\mathbf{b}_{j_{\epsilon}}\right|<\frac{\epsilon}{2}$. Thus

$$
\begin{aligned}
h_{\text {top }}\left(T, \underset{|\mathbf{b}-\mathbf{a}|<\epsilon}{\bigcup} E_{\boldsymbol{\Phi}}(\mathbf{b})\right) & \geq h_{\text {top }}\left(T, \bigcup_{\left|\mathbf{b}-\mathbf{b}_{j_{\epsilon}}\right|<\frac{\epsilon}{2}} E_{\boldsymbol{\Phi}}(\mathbf{b})\right) \\
& \geq P_{\boldsymbol{\Phi}}\left(\mathbf{t}_{j_{\epsilon}}\right)-\mathbf{b}_{j_{\epsilon}} \cdot \mathbf{t}_{j_{\epsilon}} \geq P_{\boldsymbol{\Phi}}\left(\mathbf{t}_{j_{\epsilon}}\right)-\mathbf{a} \cdot \mathbf{t}_{j_{\epsilon}} \\
& \geq \inf _{\mathbf{q} \in \mathbb{R}_{+}^{k}}\left\{P_{\boldsymbol{\Phi}}(\mathbf{q})-\mathbf{a} \cdot \mathbf{q}\right\},
\end{aligned}
$$

and hence

$$
\lim _{\epsilon \rightarrow 0} h_{\text {top }}\left(T, \underset{|\mathbf{b}-\mathbf{a}|<\epsilon}{\bigcup} E_{\boldsymbol{\Phi}}(\mathbf{b})\right) \geq \inf _{\mathbf{q} \in \mathbb{R}_{+}^{k}}\left\{P_{\boldsymbol{\Phi}}(\mathbf{q})-\mathbf{a} \cdot \mathbf{q}\right\} .
$$

Meanwhile, the upper bound follows from Corollary 4.5. This finishes the proof of part (i).

To show (ii), we first prove the following upper bound:

$$
\lim _{\epsilon \rightarrow 0} \sup \left\{h_{\mu}(T): \mu \in \mathcal{M}(X, T),\left|\Phi_{*}(\mu)-\mathbf{a}\right|<\epsilon\right\} \leq \inf _{\mathbf{q} \in \mathbb{R}_{+}^{k}}\left\{P_{\mathbf{\Phi}}(\mathbf{q})-\mathbf{a} \cdot \mathbf{q}\right\}
$$

for any $\mathbf{a}=\left(a_{1}, \ldots, a_{k}\right) \in \mathbb{R}^{k}$, where we take the convention $\sup \emptyset=-\infty$. To see it, let $\mathbf{q}=\left(q_{1}, \ldots, q_{k}\right) \in \mathbb{R}_{+}^{k}$ and $\epsilon>0$. Then by Theorem 3.1, for any $\mu \in \mathcal{M}(X, T)$ satisfying $\left|\Phi_{*}(\mu)-\mathbf{a}\right|<\epsilon$,

$$
h_{\mu}(T) \leq P_{\mathbf{\Phi}}(\mathbf{q})-\mathbf{q} \cdot \mathbf{\Phi}_{*}(\mu) \leq P_{\mathbf{\Phi}}(\mathbf{q})-\sum_{i=1}^{k}\left(a_{i}-\epsilon\right) q_{i} .
$$

That is, $\sup \left\{h_{\mu}(T): \mu \in \mathcal{M}(X, T),\left|\mathbf{\Phi}_{*}(\mu)-\mathbf{a}\right|<\epsilon\right\} \leq P_{\mathbf{\Phi}}(\mathbf{q})-\sum_{i=1}^{k}\left(a_{i}-\epsilon\right) q_{i}$. Letting $\epsilon \rightarrow 0$ yields (4.15). 
Now we prove the lower bound. Assume $\mathbf{a} \in \partial P_{\boldsymbol{\Phi}}(\mathbf{t})$ for some $\mathbf{t} \in \mathbb{R}_{+}^{k}$. Let $\epsilon>0$. Then by Minkowski's Theorem (cf. Section 2.3), there exist $\mathbf{a}_{j} \in \partial^{e} P_{\mathbf{\Phi}}(\mathbf{t})$ and $\lambda_{j} \in[0,1]$, $j=1, \ldots, k+1$, such that $\sum_{j=1}^{k+1} \lambda_{j}=1$ and

$$
\mathbf{a}=\sum_{j=1}^{k+1} \lambda_{j} \mathbf{a}_{j} .
$$

By Lemma 4.7, there exist $\nu_{j} \in \mathcal{E}(X, T), j=1, \ldots, k+1$ such that

$$
\left|\boldsymbol{\Phi}_{*}\left(\nu_{j}\right)-\mathbf{a}_{j}\right|<\epsilon, \quad\left|h_{\nu_{i}}(T)-\left(P_{\boldsymbol{\Phi}}(\mathbf{t})-\mathbf{a}_{j} \cdot \mathbf{t}\right)\right|<\epsilon .
$$

Set $\nu=\sum_{j=1}^{k+1} \lambda_{j} \nu_{j}$. Then $\nu \in \mathcal{M}(X, T)$ and

$$
\boldsymbol{\Phi}_{*}(\nu)=\sum_{j=1}^{k+1} \lambda_{j} \boldsymbol{\Phi}_{*}\left(\nu_{j}\right), \quad h_{\nu}(T)=\sum_{j=1}^{k+1} \lambda_{j} h_{\nu_{j}}(T) .
$$

Combining these with (4.16) and (4.17) yields

$$
\left|\boldsymbol{\Phi}_{*}(\nu)-\mathbf{a}\right|<\epsilon, \quad\left|h_{\nu}(T)-\left(P_{\boldsymbol{\Phi}}(\mathbf{t})-\mathbf{a} \cdot \mathbf{t}\right)\right|<\epsilon .
$$

Thus $\sup \left\{h_{\mu}(T): \mu \in \mathcal{M}(X, T),\left|\Phi_{*}(\mu)-\mathbf{a}\right|<\epsilon\right\} \geq h_{\nu}(T) \geq P_{\boldsymbol{\Phi}}(\mathbf{t})-\mathbf{a} \cdot \mathbf{t}-\epsilon$. Letting $\epsilon \rightarrow 0$ yields the desired lower bound

$$
\begin{aligned}
& \lim _{\epsilon \rightarrow 0} \sup \left\{h_{\mu}(T): \mu \in \mathcal{M}(X, T),\left|\mathbf{\Phi}_{*}(\mu)-\mathbf{a}\right|<\epsilon\right\} \\
& \geq P_{\boldsymbol{\Phi}}(\mathbf{t})-\mathbf{a} \cdot \mathbf{t}=\inf _{\mathbf{q} \in \mathbb{R}_{+}^{k}}\left\{P_{\boldsymbol{\Phi}}(\mathbf{q})-\mathbf{a} \cdot \mathbf{q}\right\} .
\end{aligned}
$$

In the end, we assume $\mathbf{a} \in \operatorname{cl}_{+}\left(\partial P_{\boldsymbol{\Phi}}\right)$. Then there exist $\mathbf{t}_{j} \in \mathbb{R}_{+}^{k}$ and $\mathbf{b}_{j} \in \partial^{e} P_{\boldsymbol{\Phi}}\left(\mathbf{t}_{j}\right), j \in \mathbb{N}$ such that $\mathbf{a} \geq \mathbf{b}_{j}$ and $\lim _{j \rightarrow \infty} \mathbf{b}_{j}=\mathbf{a}$. Let $\epsilon>0$. There exists a large $j_{\epsilon}$ such that $\left|\mathbf{a}-\mathbf{b}_{j_{\epsilon}}\right|<\frac{\epsilon}{2}$. Thus

$$
\begin{aligned}
\sup & \left\{h_{\mu}(T): \mu \in \mathcal{M}(X, T),\left|\boldsymbol{\Phi}_{*}(\mu)-\mathbf{a}\right|<\epsilon\right\} \\
& \geq \sup \left\{h_{\mu}(T): \mu \in \mathcal{M}(X, T),\left|\Phi_{*}(\mu)-\mathbf{b}_{j_{\epsilon}}\right|<\frac{\epsilon}{2}\right\} \\
\geq & P_{\boldsymbol{\Phi}}\left(\mathbf{t}_{j_{\epsilon}}\right)-\mathbf{b}_{j_{\epsilon}} \cdot \mathbf{t}_{j_{\epsilon}} \geq P_{\boldsymbol{\Phi}}\left(\mathbf{t}_{j_{\epsilon}}\right)-\mathbf{a} \cdot \mathbf{t}_{j_{\epsilon}} \\
\geq & \inf _{\mathbf{q} \in \mathbb{R}_{+}^{k}}\left\{P_{\boldsymbol{\Phi}}(\mathbf{q})-\mathbf{a} \cdot \mathbf{q}\right\},
\end{aligned}
$$

and hence

$$
\lim _{\epsilon \rightarrow 0} \sup \left\{h_{\mu}(T): \mu \in \mathcal{M}(X, T),\left|\boldsymbol{\Phi}_{*}(\mu)-\mathbf{a}\right|<\epsilon\right\} \geq \inf _{\mathbf{q} \in \mathbb{R}_{+}^{k}}\left\{P_{\mathbf{\Phi}}(\mathbf{q})-\mathbf{a} \cdot \mathbf{q}\right\} .
$$

This finishes the proof of part (ii).

To show part (iii), let $A=\left\{\mathbf{a} \in \mathbb{R}^{k}: \mathbf{a}=\mathbf{\Phi}_{*}(\mu)\right.$ for some $\left.\mu \in \mathcal{M}(X, T)\right\}$. Clearly, $A$ is non-empty and convex. Define $g: A \rightarrow \mathbb{R}$ by

$$
g(\mathbf{a})=\sup \left\{h_{\mu}(T): \mu \in \mathcal{M}(X, T), \mathbf{\Phi}_{*}(\mu)=\mathbf{a}\right\} .
$$


Then $g$ is a real-valued concave function on $A$. Take

$$
W(\mathbf{x})=\sup \{g(\mathbf{a})+\mathbf{a} \cdot \mathbf{x}: \mathbf{a} \in A\}, \quad \forall \mathbf{x} \in \mathbb{R}^{k} .
$$

Apply Corollary 2.5 to obtain

$$
\inf \left\{W(\mathbf{x})-\mathbf{a} \cdot \mathbf{x}: \mathbf{x} \in \mathbb{R}^{k}\right\}=g(\mathbf{a}), \quad \forall \mathbf{a} \in \operatorname{ri}(A) .
$$

However, by Theorem 3.1, we have $P_{\mathbf{\Phi}}(\mathbf{q})=W(\mathbf{q})$ for all $\mathbf{q} \in \mathbb{R}_{+}^{k}$. Now assume that $\mathbf{a} \in \partial P_{\mathbf{\Phi}}(\mathbf{q}) \cap \operatorname{ri}(A)$ for some $\mathbf{q} \in \mathbb{R}_{+}^{k}$. Then $\mathbf{a} \in \partial W(\mathbf{q}) \cap \operatorname{ri}(A)$. Hence

$$
\begin{aligned}
g(\mathbf{a}) & =\inf \left\{W(\mathbf{x})-\mathbf{a} \cdot \mathbf{x}: \mathbf{x} \in \mathbb{R}^{k}\right\}=W(\mathbf{q})-\mathbf{a} \cdot \mathbf{q} \\
& =P_{\mathbf{\Phi}}(\mathbf{q})-\mathbf{a} \cdot \mathbf{q}=\inf \left\{P_{\mathbf{\Phi}}(\mathbf{x})-\mathbf{a} \cdot \mathbf{x}: \mathbf{x} \in \mathbb{R}_{+}^{k}\right\} .
\end{aligned}
$$

This finishes the proof of (iii) and hence the proof of Theorem 4.2,

Proof of Theorem 1.2. Here we show that Theorem 1.2 is just the one-dimensional version of Theorem 4.2. To see it, let $(X, T)$ be a TDS with $h_{\text {top }}(T)<\infty$ and let $\Phi=\left\{\log \phi_{n}\right\}_{n=1}^{\infty}$ be an asymptotically sub-additive potential on $X$ satisfying $\bar{\beta}(\Phi)>-\infty$. Let $t>0$. It is clear that $\partial^{e} P_{\Phi}(t)=\left\{P_{\Phi}^{\prime}(t-), P_{\Phi}^{\prime}(t+)\right\}$ and $\partial P_{\Phi}(t)=\left[P_{\Phi}^{\prime}(t-), P_{\Phi}^{\prime}(t+)\right]$. Thus

$$
\partial P_{\Phi}\left(\mathbb{R}_{+}\right)=\bigcup_{t>0}\left[P_{\Phi}^{\prime}(t-), P_{\Phi}^{\prime}(t+)\right] \quad \text { and } \quad \partial^{e} P_{\Phi}\left(\mathbb{R}_{+}\right)=\bigcup_{t>0}\left\{P_{\Phi}^{\prime}(t-), P_{\Phi}^{\prime}(t+)\right\} .
$$

Moreover,

$$
\operatorname{cl}_{+}\left(\partial P_{\Phi}\left(\mathbb{R}_{+}\right)\right)=\partial P_{\Phi}\left(\mathbb{R}_{+}\right) \cup\left\{P_{\Phi}^{\prime}(\infty)\right\} \text { and } \operatorname{cl}_{+}\left(\partial^{e} P_{\Phi}\left(\mathbb{R}_{+}\right)\right)=\partial^{e} P_{\Phi}\left(\mathbb{R}_{+}\right) \cup\left\{P_{\Phi}^{\prime}(\infty)\right\} .
$$

Furthermore, by Lemma $\mathrm{A.3}(4), q \bar{\beta}(\Phi) \leq P_{\Phi}(q) \leq h_{\mathrm{top}}(T)+q \bar{\beta}(\Phi)$, from which we obtain $P_{\Phi}^{\prime}(\infty):=\lim _{q \rightarrow \infty} P_{\Phi}(q) / q=\bar{\beta}(\Phi)$. By the way, applying Theorem 4.2 (ii), for each $a \in$ $\partial P_{\Phi}(t)$ and any $\epsilon>0$, there exists $\mu \in \mathcal{M}(X, T)$ such that $\left|\Phi_{*}(\mu)-a\right|<\epsilon$. It implies that

$$
\left(\lim _{t \rightarrow 0+} P_{\Phi}^{\prime}(t-), P_{\Phi}^{\prime}(\infty)\right) \subseteq \operatorname{int}(A)=\operatorname{ri}(A),
$$

where $A:=\left\{a \in \mathbb{R}: a=\Phi_{*}(\mu)\right.$ for some $\left.\mu \in \mathcal{M}(X, T)\right\}$. According to (4.19)-(4.21), Theorem 1.2 just follows from Theorem 4.2 ,

4.3. A high-dimensional version of Theorem 1.3, Let $\Phi_{i}=\left\{\log \phi_{n, i}\right\}_{n=1}^{\infty}(i=1, \ldots, k)$ be asymptotically sub-additive potentials on a $\operatorname{TDS}(X, T)$. Let $\boldsymbol{\Phi}=\left(\Phi_{1}, \ldots, \Phi_{k}\right)$. For $\delta>0$, we define

$$
\mathrm{cl}_{+}^{\delta}\left(\partial P_{\boldsymbol{\Phi}}\left(\mathbb{R}_{+}^{k}\right)\right):=\mathrm{cl}_{+}\left(\underset{\left\{\mathbf{t} \in \mathbb{R}_{+}^{k}: t_{i} \geq \delta, i=1,2, \cdots, k\right\}}{\bigcup} \partial P_{\boldsymbol{\Phi}}(\mathbf{t})\right),
$$

where $\mathrm{cl}_{+}(A)$ is defines as in (4.5).

The following theorem is a high dimensional version of Theorem 1.3 .

Theorem 4.8. Assume $h_{\mathrm{top}}(T)<\infty, \bar{\beta}(\mathbf{\Phi})>-\infty$, and that the entropy map $\mu \mapsto h_{\mu}(T)$ is upper semi-continuous on $\mathcal{M}(X, T)$. Then 
(i) For any $\mathbf{t} \in \mathbb{R}_{+}^{k}$, if $\mathbf{a} \in \partial^{e} P_{\boldsymbol{\Phi}}(\mathbf{t})$, then $E_{\boldsymbol{\Phi}}(\mathbf{a}) \neq \emptyset$ and

$$
h_{\text {top }}\left(T, E_{\boldsymbol{\Phi}}(\mathbf{a})\right)=\inf _{\mathbf{q} \in \mathbb{R}_{+}^{k}}\left\{P_{\boldsymbol{\Phi}}(\mathbf{q})-\mathbf{a} \cdot \mathbf{q}\right\}=P_{\boldsymbol{\Phi}}(\mathbf{t})-\mathbf{a} \cdot \mathbf{t} .
$$

(ii) For $\mathbf{a} \in \bigcup_{\delta>0} \operatorname{cl}_{+}^{\delta}\left(\partial P_{\mathbf{\Phi}}\left(\mathbb{R}_{+}^{k}\right)\right)$,

$$
\inf _{\mathbf{q} \in \mathbb{R}_{+}^{k}}\left\{P_{\boldsymbol{\Phi}}(\mathbf{q})-\mathbf{a} \cdot \mathbf{q}\right\}=\max \left\{h_{\mu}(T): \mu \in \mathcal{M}(X, T), \boldsymbol{\Phi}_{*}(\mu)=\mathbf{a}\right\} .
$$

(iii) If $\mathbf{t} \in \mathbb{R}_{+}^{k}$ such that $\mathbf{t} \cdot \boldsymbol{\Phi}$ has a unique equilibrium state $\mu_{\mathbf{t}} \in \mathcal{M}(X, T)$, then $\mu_{\mathbf{t}}$ is ergodic, $P_{\boldsymbol{\Phi}}^{\prime}(\mathbf{t})=\boldsymbol{\Phi}_{*}\left(\mu_{\mathbf{t}}\right), E_{\boldsymbol{\Phi}}\left(P_{\boldsymbol{\Phi}}^{\prime}(\mathbf{t})\right) \neq \emptyset$ and $h_{\mathrm{top}}\left(T, E_{\boldsymbol{\Phi}}\left(P_{\boldsymbol{\Phi}}^{\prime}(\mathbf{t})\right)\right)=h_{\mu_{\mathbf{t}}}(T)$.

Proof. We first prove (i). Fix $\mathbf{t} \in \mathbb{R}_{+}^{k}$. By Theorem 3.3, $\mathcal{I}(\mathbf{\Phi}, \mathbf{t})$ is a non-empty compact convex subset of $\mathcal{M}(X, T)$. We claim that for any $\mathbf{b} \in \mathbb{R}^{k}$ the set

$$
\mathcal{I}_{\mathbf{b}}(\boldsymbol{\Phi}, \mathbf{t}):=\left\{\nu \in \mathcal{I}(\boldsymbol{\Phi}, \mathbf{t}): \boldsymbol{\Phi}_{*}(\nu)=\mathbf{b}\right\}
$$

is compact and convex. The convexity is clear. To show the compactness, assume that $\left\{\nu_{n}\right\} \subset \mathcal{I}_{\mathbf{b}}(\mathbf{\Phi}, \mathbf{t})$ and $\nu_{n}$ converges to $\nu$ in $\mathcal{M}(X, T)$. Then by the upper semi-continuity of $h_{(\cdot)}(T)$ and $\left(\Phi_{i}\right)_{*}(\cdot), i=1, \ldots, k$, we have

$$
h_{\nu}(T)+\mathbf{t} \cdot \mathbf{\Phi}_{*}(\nu) \geq \lim _{n \rightarrow \infty} h_{\nu_{n}}(T)+\mathbf{t} \cdot \mathbf{\Phi}_{*}\left(\nu_{n}\right)=P_{\mathbf{\Phi}}(\mathbf{t}) .
$$

By Theorem 3.1, $\nu \in \mathcal{I}(\boldsymbol{\Phi}, \mathbf{t})$ and furthermore, $h_{\nu}(T)=h_{\nu_{n}}(T)=P_{\boldsymbol{\Phi}}(\mathbf{t})-\mathbf{t} \cdot \mathbf{b}$ and $\boldsymbol{\Phi}_{*}(\nu)=\boldsymbol{\Phi}_{*}\left(\nu_{n}\right)=\mathbf{b}$. This is, $\nu \in \mathcal{I}_{\mathbf{b}}(\boldsymbol{\Phi}, \mathbf{t})$. Hence $\mathcal{I}_{\mathbf{b}}(\boldsymbol{\Phi}, \mathbf{t})$ is compact. This finishes the proof of the claim.

Now let $\mathbf{a} \in \partial^{e} P_{\boldsymbol{\Phi}}(\mathbf{t})$. By Theorem 3.3, the set $\mathcal{I}_{\mathbf{a}}(\boldsymbol{\Phi}, \mathbf{t})$ is non-empty. We are going to show further that $\mathcal{I}_{\mathbf{a}}(\boldsymbol{\Phi}, \mathbf{t})$ contains at least one ergodic measure. Since $\mathcal{I}_{\mathbf{a}}(\boldsymbol{\Phi}, \mathbf{t})$ is a non-empty compact convex subset of $\mathcal{M}(X, T)$, by the Krein-Milman theorem (c.f. [17, p. 146]), it contains at least one extreme point, denoted by $\nu$. Let $\nu=p \nu_{1}+(1-p) \nu_{2}$ for some $0<p<1$ and $\nu_{1}, \nu_{2} \in \mathcal{M}(X, T)$. Then

$$
\begin{aligned}
P_{\boldsymbol{\Phi}}(\mathbf{t}) & =h_{\nu}(T)+\mathbf{t} \cdot \boldsymbol{\Phi}_{*}(\nu) \\
& =p\left(h_{\nu_{1}}(T)+\mathbf{t} \cdot \boldsymbol{\Phi}_{*}\left(\nu_{1}\right)\right)+(1-p)\left(h_{\nu_{2}}(T)+\mathbf{t} \cdot \boldsymbol{\Phi}_{*}\left(\nu_{2}\right)\right) .
\end{aligned}
$$

By Theorem 3.1, $\nu_{1}, \nu_{2} \in \mathcal{I}(\mathbf{\Phi}, \mathbf{t})$. By Theorem 3.3, $\boldsymbol{\Phi}_{*}\left(\nu_{1}\right), \boldsymbol{\Phi}_{*}\left(\nu_{2}\right) \in \partial P_{\boldsymbol{\Phi}}(\mathbf{t})$. Moreover, note that $\mathbf{a}=\boldsymbol{\Phi}_{*}(\nu)=p \boldsymbol{\Phi}_{*}\left(\nu_{1}\right)+(1-p) \boldsymbol{\Phi}_{*}\left(\nu_{2}\right)$, we have $\boldsymbol{\Phi}_{*}\left(\nu_{1}\right)=\boldsymbol{\Phi}_{*}\left(\nu_{2}\right)=\mathbf{a}$ since $\mathbf{a} \in \partial^{e} P_{\boldsymbol{\Phi}}(\mathbf{t})$. That is, $\nu_{1}, \nu_{2} \in \mathcal{I}_{\mathbf{a}}(\boldsymbol{\Phi}, \mathbf{t})$. Since $\nu$ is an extreme point of $\mathcal{I}_{\mathbf{a}}(\boldsymbol{\Phi}, \mathbf{t})$, we have $\nu_{1}=\nu_{2}=\nu$. It follows that $\nu$ is an extreme point of $\mathcal{M}(X, T)$, i.e., $\nu$ is ergodic. By Proposition A.1(1), we have $\nu\left(E_{\boldsymbol{\Phi}}(\mathbf{a})\right)=1$, and thus by Proposition 2.1(3),

$$
h_{\text {top }}\left(T, E_{\boldsymbol{\Phi}}(\mathbf{a})\right) \geq h_{\nu}(T)=P_{\boldsymbol{\Phi}}(\mathbf{t})-\mathbf{t} \cdot \mathbf{a}=\inf _{\mathbf{q} \in \mathbb{R}_{+}^{k}}\left\{P_{\boldsymbol{\Phi}}(\mathbf{q})-\mathbf{q} \cdot \mathbf{a}\right\} .
$$

However by Corollary 4.5, the upper bound $h_{\text {top }}\left(T, E_{\boldsymbol{\Phi}}(\mathbf{a})\right) \leq \inf _{\mathbf{q} \in \mathbb{R}_{+}^{k}}\left\{P_{\boldsymbol{\Phi}}(\mathbf{q})-\mathbf{q} \cdot \mathbf{a}\right\}$ is generic. Thus we have the equality

$$
h_{\mathrm{top}}\left(T, E_{\boldsymbol{\Phi}}(\mathbf{a})\right)=h_{\nu}(T)=P_{\boldsymbol{\Phi}}(\mathbf{t})-\mathbf{t} \cdot \mathbf{a}=\inf _{\mathbf{q} \in \mathbb{R}_{+}^{k}}\left\{P_{\boldsymbol{\Phi}}(\mathbf{q})-\mathbf{q} \cdot \mathbf{a}\right\} .
$$


This finishes the proof of part (i).

To show (ii), by Theorem 4.2(ii) we need only to show that for $\mathbf{a} \in \mathrm{cl}_{+}^{\delta}\left(\partial P_{\mathbf{\Phi}}\right)$,

$$
\inf _{\mathbf{q} \in \mathbb{R}_{+}^{k}}\left\{P_{\boldsymbol{\Phi}}(\mathbf{q})-\mathbf{a} \cdot \mathbf{q}\right\} \leq \max \left\{h_{\mu}(T): \mu \in \mathcal{M}(X, T), \boldsymbol{\Phi}_{*}(\mu)=\mathbf{a}\right\} .
$$

To see it, we first assume that $\mathbf{a} \in \partial P_{\boldsymbol{\Phi}}(\mathbf{t})$ for some $\mathbf{t} \in \mathbb{R}_{+}^{k}$. By Theorem 4.2 (ii), there exists $\left(\nu_{j}\right) \subset \mathcal{M}(X, T)$ such that

$$
\lim _{j \rightarrow \infty} \boldsymbol{\Phi}_{*}\left(\nu_{j}\right)=\mathbf{a} \text { and } \limsup _{j \rightarrow \infty} h_{\nu_{j}}(T) \geq \inf _{\mathbf{q} \in \mathbb{R}_{+}^{k}}\left\{P_{\boldsymbol{\Phi}}(\mathbf{q})-\mathbf{a} \cdot \mathbf{q}\right\} .
$$

Extract a subsequence if necessary so that $\lim _{j \rightarrow \infty} \nu_{j}=\nu$ for some $\nu \in \mathcal{M}(X, T)$. Then

$$
h_{\nu}(T) \geq \limsup _{j \rightarrow \infty} h_{\nu_{j}}(T) \geq \inf _{\mathbf{q} \in \mathbb{R}_{+}^{k}}\left\{P_{\boldsymbol{\Phi}}(\mathbf{q})-\mathbf{a} \cdot \mathbf{q}\right\}=P_{\boldsymbol{\Phi}}(\mathbf{t})-\mathbf{a} \cdot \mathbf{t}
$$

and $\boldsymbol{\Phi}_{*}(\nu) \geq \limsup \sup _{j \rightarrow \infty} \boldsymbol{\Phi}_{*}\left(\nu_{j}\right)=\mathbf{a}$ by the upper-semi continuity of $h_{(\cdot)}(T)$ and $\left(\Phi_{i}\right)_{*}(\cdot)$. Hence

$$
h_{\nu}(T) \geq P_{\boldsymbol{\Phi}}(\mathbf{t})-\mathbf{a} \cdot \mathbf{t} \geq P_{\boldsymbol{\Phi}}(\mathbf{t})-\boldsymbol{\Phi}_{*}(\nu) \cdot \mathbf{t} \geq h_{\nu}(T),
$$

which implies $\boldsymbol{\Phi}_{*}(\nu)=\mathbf{a}$ and $h_{\nu}(T)=P_{\boldsymbol{\Phi}}(\mathbf{t})-\mathbf{a} \cdot \mathbf{t}$.

Next we assume that $\mathbf{a} \in \operatorname{cl}_{+}^{\delta}\left(\partial P_{\boldsymbol{\Phi}}\left(\mathbb{R}_{+}^{k}\right)\right)$ for some $\delta>0$. Then there exists a sequence $\left(\mathbf{t}_{j}\right) \in \mathbb{R}_{+}^{k}$ such that each entry of $\mathbf{t}_{j}$ is greater than $\delta$, and there exists $\mathbf{a}_{j} \in \partial^{e} P_{\boldsymbol{\Phi}}\left(\mathbf{t}_{j}\right)$ for each $j$ such that $\mathbf{a} \geq \mathbf{a}_{j}$ and $\lim _{j \rightarrow \infty} \mathbf{a}_{j}=\mathbf{a}$. By the above discussion, for each $j \in \mathbb{N}$ there exists $\mu_{j} \in \mathcal{M}(X, T)$ such that $\boldsymbol{\Phi}_{*}\left(\mu_{j}\right)=\mathbf{a}_{j}$ and $h_{\mu_{j}}(T)=P_{\boldsymbol{\Phi}}\left(\mathbf{t}_{j}\right)-\mathbf{a}_{j} \cdot \mathbf{t}_{j}$. Extract a subsequence if necessary so that $\lim _{j \rightarrow \infty} \mu_{j}=\mu$ for some $\mu \in \mathcal{M}(X, T)$. Thus $\boldsymbol{\Phi}_{*}(\mu) \geq \lim _{j \rightarrow \infty} \boldsymbol{\Phi}_{*}\left(\mu_{j}\right)=\mathbf{a}$ and

$$
\begin{aligned}
h_{\mu}(T) & \geq \limsup _{j \rightarrow \infty} h_{\mu_{j}}(T)=\limsup _{j \rightarrow \infty}\left(P_{\boldsymbol{\Phi}}\left(\mathbf{t}_{j}\right)-\mathbf{a}_{j} \cdot \mathbf{t}_{j}\right) \\
& \geq \limsup _{j \rightarrow \infty}\left(P_{\boldsymbol{\Phi}}\left(\mathbf{t}_{j}\right)-\mathbf{a} \cdot \mathbf{t}_{j}\right) \\
& \geq \limsup _{j \rightarrow \infty}\left(h_{\mu}(T)+\left(\boldsymbol{\Phi}_{*}(\mu)-\mathbf{a}\right) \cdot \mathbf{t}_{\mathbf{j}}\right) \\
& \geq h_{\mu}(T)+\sum_{i=1}^{k}\left(\left(\Phi_{i}\right)_{*}(\mu)-a_{i}\right) \delta .
\end{aligned}
$$

This implies that $\boldsymbol{\Phi}_{*}(\mu)=\mathbf{a}$ and

$$
h_{\mu}(T) \geq \limsup _{j \rightarrow \infty}\left(P_{\mathbf{\Phi}}\left(\mathbf{t}_{j}\right)-\mathbf{a} \cdot \mathbf{t}_{j}\right) \geq \inf _{\mathbf{q} \in \mathbb{R}_{+}^{k}}\left\{P_{\mathbf{\Phi}}(\mathbf{q})-\mathbf{a} \cdot \mathbf{q}\right\} .
$$

This finishes the proof of part (ii).

Now we turn to prove (iii). We assume $\mathbf{t} \in \mathbb{R}_{+}^{k}$ such that $\mathbf{t} \cdot \boldsymbol{\Phi}$ has a unique equilibrium state $\mu_{\mathbf{t}}$. By Theorem 3.3, $\partial P_{\boldsymbol{\Phi}}(\mathbf{t})=\left\{\boldsymbol{\Phi}_{*}\left(\mu_{t}\right)\right\}$. Now (iii) comes from parts (i) and (ii) of the theorem. 
Proof of Theorem 1.3. It follows directly from Theorem 4.8, using the fact that in the onedimensional case, $\partial^{e} P_{\Phi}(t)=\left\{P_{\Phi}^{\prime}(t+), P_{\Phi}^{\prime}(t-)\right\}$ for $t>0$ and

$$
\bigcup_{\delta>0} \mathrm{cl}_{+}^{\delta}\left(\partial P_{\Phi}\left(\mathbb{R}_{+}\right)\right)=\bigcup_{t>0}\left[P_{\Phi}^{\prime}(t-), P_{\Phi}^{\prime}(\infty)\right]
$$

Remark 4.9. Theorem 1.3(i) has a nice application in the multifractal analysis of measures on symbolic spaces. Let $\mu$ be a fully supported Borel probability measure on the one-sided full shift space $(\Sigma, \sigma)$ over a finite alphabet. Assume in addition that

$$
\mu\left(I_{n+m}(x)\right) \leq C \mu\left(I_{n}(x)\right) \mu\left(I_{m}\left(\sigma^{n} x\right)\right), \quad \forall x \in \Sigma, n, m \in \mathbb{N},
$$

where $C>0$ is a constant and $I_{n}(y)$ denotes the $n$-th cylinder in $\Sigma$ containing $y$. Let $\Phi$ be a potential on $\Sigma$ given by $\Phi=\left\{\log \mu\left(I_{n}(x)\right)\right\}_{n=1}^{\infty}$. By applying a general multifractal result of Ben Nasr [10], Testud [48] obtained that (formulated in our terminologies as)

$$
h_{\mathrm{top}}\left(\sigma, E_{\Phi}(\alpha)\right)=\inf \left\{P_{\Phi}(q)-\alpha q: q>0\right\} \quad \text { whenever } \alpha=P^{\prime}(t) \text { for some } t>0,
$$

provided that $P_{\Phi}^{\prime}(t)$ exists at $t$. However by Proposition $A .5(i), \Phi$ is asymptotically subadditive, hence by Theorem 1.3(i), the above variational relation actually holds for any $\alpha=$ $P_{\Phi}^{\prime}(t+)$ and $\alpha=P_{\Phi}^{\prime}(t-)$ for each $t>0$. Furthermore, the constant $C$ in (4.23) can be replaced by $C_{n}$, where $\left(C_{n}\right)$ is a sequence of positive numbers satisfying $\lim _{n \rightarrow \infty}(1 / n) \log C_{n}=$ 0 (cf. Remark A.6 (iii)).

\subsection{Lyapunov spectrum for certain sub-additive potentials on symbolic spaces.}

In this subsection, we assume that $(X, T)$ is the one-sided full shift over an finite set $\{1,2, \ldots, m\}$. That is, $X=\{1, \ldots, m\}^{\mathbb{N}}$ endowed with the standard metric $d(x, y)=2^{-n}$ for $x=\left(x_{i}\right)_{i=1}^{\infty}$ and $\left(y_{i}\right)_{i=1}^{\infty}$, where $n$ is the largest integer so that $x_{j}=y_{j}$ for $1 \leq j \leq n$, and $T$ is the shift map given by $\left(x_{i}\right)_{i=1}^{\infty} \mapsto\left(x_{i+1}\right)_{i=1}^{\infty}$.

Let $X^{*}$ denote the collection of finite words over $\{1, \ldots, m\}$, i.e., $X^{*}=\bigcup_{i=1}^{\infty}\{1, \ldots, m\}^{i}$. Assume that $\phi: X^{*} \rightarrow[0, \infty)$ is a map (not identically equal to 0 ) satisfying the following two assumptions:

(H1) $\phi(I J) \leq \phi(I) \phi(J)$ for any $I, J \in X^{*}$;

(H2) There exist a sequence of positive integers $\left(t_{n}\right)$ and a sequence of positive numbers $\left(c_{n}\right)$ with $\lim _{n \rightarrow \infty} t_{n} / n=0=\lim _{n \rightarrow \infty}(1 / n) \log c_{n}$, such that for each $I, J \in X^{*}$ with lengths $|I| \geq n,|J| \geq n$, there exists $K \in X^{*}$ with $|K| \leq t_{n}$ so that

$$
\phi(I K J) \geq c_{n} \phi(I) \phi(J) .
$$

Let $\Phi=\left(\log \phi_{n}\right)$ be a potential on $X$ given by $\phi_{n}(x)=\phi\left(x_{1} \ldots x_{n}\right)$ for $x=\left(x_{i}\right)_{i=1}^{\infty}$. It is clear that $\Phi$ is sub-additive. Denote

$$
P(q):=\limsup _{n \rightarrow \infty} \frac{1}{n} \log \sum \phi(I)^{q},
$$


where the sum is taken over the set of $I \in\{1, \ldots, m\}^{n}$ with $\phi(I)>0$. It is clear that $P(q)=P(T, q \Phi)$ for $q>0$. Although $\Phi$ is not necessary to be asymptotically additive, we still have the following rather complete result, as an analogue of our recent work [24] on the norm of matrix products.

Theorem 4.10. Let $\Phi$ be given as above. Assume that $P(q) \in \mathbb{R}$ for each $q<0$. Then $\left\{\alpha \in \mathbb{R}: E_{\Phi}(\alpha) \neq \emptyset\right\}=\mathbb{R} \cap[a, b]$, where $a=\lim _{n \rightarrow-\infty} P(q) / q$ and $b=\lim _{n \rightarrow \infty} P(q) / q$. Furthermore, for $\alpha \in \mathbb{R} \cap[a, b]$,

$$
h_{\text {top }}(T, E(\alpha))=\inf \{P(q)-a q: q \in \mathbb{R}\} .
$$

Proof of Theorem 4.10. Take a slight modification of the proof of Theorem 1.1 in [24].

We remark that under the condition of the above theorem, we always have $b \in \mathbb{R}$. However it is possible that $a=-\infty$.

There are some natural maps $\phi: X^{*} \rightarrow[0, \infty)$ which satisfy the assumptions (H1)(H2). For example, if $\left\{M_{i}\right\}_{i=1}^{m}$ is a family of $d \times d$ real matrices so that there is no trivial proper linear subspace $V \subset \mathbb{R}^{d}$ with $M_{i} V \subseteq V$ for all $i$, then the map $\phi$ defined by $\phi\left(x_{1} \ldots x_{n}\right)=\left\|M_{x_{1}} \ldots M_{x_{n}}\right\|$ satisfies (H1)-(H2) (see [24, 19]). More generally, the singular value functions for $M_{x_{1}} \ldots M_{x_{n}}$ also satisfy (H1)-(H2) when $\left\{M_{i}\right\}_{i=1}^{m}$ satisfies further mild irreducibility conditions (see [19]).

\section{The MUlTiFRACTAL FORMALISM FOR ASYMPTOTICALLY ADDITIVE POTENTIALS}

Let $(X, T)$ be a TDS. Let $k \in \mathbb{N}$ and let $\Phi_{i}=\left\{\log \phi_{n, i}\right\}_{n=1}^{\infty}, \Psi_{i}=\left\{\log \psi_{n, i}\right\}_{n=1}^{\infty}(i=$ $1,2, \cdots, k)$ be asymptotically additive potentials on $(X, T)$. Furthermore assume

$$
\psi_{n, i}(x) \geq C(1+\delta)^{n} \quad(i \in\{1,2, \cdots, k\}, n \in \mathbb{N}, x \in X)
$$

for some constants $C, \delta>0$. This assumption guarantees that $\left(\Psi_{i}\right)_{*}(\mu) \neq 0(i=1,2, \cdots, k)$ for each $\mu \in \mathcal{M}(X, T)$.

For $\mathbf{a}=\left(a_{1}, \ldots, a_{k}\right) \in \mathbb{R}^{k}$, denote

$$
E(\mathbf{a}):=\left\{x \in X: \lim _{n \rightarrow \infty} \frac{\log \phi_{n, i}(x)}{\log \psi_{n, i}(x)}=a_{i} \text { for } 1 \leq i \leq k\right\} .
$$

In this section, we shall study the multifractal structure of $E(\mathbf{a})$.

For $\mu \in \mathcal{M}(X, T)$, the set $G_{\mu}$ of $\mu$-generic points is defined by

$$
G_{\mu}:=\left\{x \in X: \frac{1}{n} \sum_{j=0}^{n-1} \delta_{T^{j} x} \rightarrow \mu \text { in the weak* topology as } n \rightarrow \infty\right\},
$$

where $\delta_{y}$ denotes the probability measure whose support is the single point $y$. Bowen [12] showed that $h_{\mathrm{top}}\left(T, G_{\mu}\right) \leq h_{\mu}(T)$ for any $\mu \in \mathcal{M}(X, T)$. 
Definition 5.1. A TDS $(X, T)$ is called to be saturated if for any $\mu \in \mathcal{M}(X, T)$, we have $G_{\mu} \neq \emptyset$ and $h_{\mathrm{top}}\left(T, G_{\mu}\right)=h_{\mu}(T)$.

It was shown independently in [22, 44] that if a TDS satisfies the specification property (or a weaker form), then it is saturated. The main result in this section is the following.

Theorem 5.2. Let $(X, T)$ be a TDS and let $\Phi_{i}, \Psi_{i}(i=1,2, \cdots, k)$ be asymptotically additive potentials on $X$ satisfying the assumption (5.1). Let $\Omega \subset \mathbb{R}^{k}$ be the range of the following map from $\mathcal{M}(X, T)$ to $\mathbb{R}^{k}$ :

For $\mathbf{a} \in \Omega$, write

$$
\mu \rightarrow\left(\frac{\left(\Phi_{1}\right)_{*}(\mu)}{\left(\Psi_{1}\right)_{*}(\mu)}, \frac{\left(\Phi_{2}\right)_{*}(\mu)}{\left(\Psi_{2}\right)_{*}(\mu)}, \cdots, \frac{\left(\Phi_{k}\right)_{*}(\mu)}{\left(\Psi_{k}\right)_{*}(\mu)}\right) .
$$

$$
H(\mathbf{a})=\sup \left\{h_{\mu}(T): \mu \in \mathcal{M}(X, T),\left(\Phi_{i}\right)_{*}(\mu)=a_{i}\left(\Psi_{i}\right)_{*}(\mu) \text { for } i=1,2, \cdots, k\right\} .
$$

Then we have the following properties:

(i) $\left\{\mathbf{a} \in \mathbb{R}^{k}: E(\mathbf{a}) \neq \emptyset\right\} \subseteq \Omega$.

(ii) If $h_{\mathrm{top}}(T)<\infty$, then we have

$$
\mathbf{a} \in \Omega \Longleftrightarrow \inf \left\{P_{\mathbf{a}}(\mathbf{q}): \mathbf{q} \in \mathbb{R}^{k}\right\} \neq-\infty \Longleftrightarrow \inf \left\{P_{\mathbf{a}}(\mathbf{q}): \mathbf{q} \in \mathbb{R}^{k}\right\} \geq 0
$$

where $P_{\mathbf{a}}(\mathbf{q}):=P\left(T, \sum_{i=1}^{k} q_{i}\left(\Phi_{i}-a_{i} \Psi_{i}\right)\right)$.

(iii) Assume that $h_{\text {top }}(T)<\infty$ and the entropy map is upper semi-continuous. Then for any $\mathbf{a} \in \Omega$,

$$
H(\mathbf{a})=\inf _{\mathbf{q} \in \mathbb{R}^{k}} P_{\mathbf{a}}(\mathbf{q})
$$

(iv) Assume that $(X, T)$ is saturated. Then $E(\mathbf{a}) \neq \emptyset$ if and only if $\mathbf{a} \in \Omega$. Furthermore

$$
h_{\text {top }}(T, E(\mathbf{a}))=H(\mathbf{a}), \quad \forall \mathbf{a} \in \Omega .
$$

We emphasize that in parts (i)-(iii) of the above theorem, we do not need to assume that $(X, T)$ is saturated.

Proof. We first prove (i). Assume that $E(\mathbf{a}) \neq \emptyset$ for some $\mathbf{a}=\left(a_{1}, \ldots, a_{k}\right) \in \mathbb{R}^{k}$. Take $x \in E(\mathbf{a})$. Denote $\mu_{x, n}=(1 / n) \sum_{j=1}^{n-1} \delta_{T^{j} x}$. Then there exists $n_{j} \uparrow \infty$ so that $\mu_{x, n_{j}} \rightarrow \mu$ for some $\mu \in \mathcal{M}(X, T)$. Apply Lemma A.4(ii) (in which we take $\nu_{n}=\delta_{x}$ ) to obtain

$$
\frac{\left(\Phi_{i}\right)_{*}(\mu)}{\left(\Psi_{i}\right)_{*}(\mu)}=\lim _{j \rightarrow \infty} \frac{\log \phi_{n_{j}, i}(x)}{\log \psi_{n_{j}, i}(x)}=a_{i}, \quad i=1, \ldots, k .
$$

Hence $\mathbf{a} \in \Omega$. This proves (i).

To show (ii), assume $h_{\text {top }}(T)<\infty$. For $\mathbf{a}=\left(a_{1}, \ldots, a_{k}\right) \in \mathbb{R}^{k}$ and $\mu \in \mathcal{M}(X, T)$, we denote

$$
\tau_{\mathbf{a}}(\mu)=\left(\left(\Phi_{1}\right)_{*}(\mu)-a_{1}\left(\Psi_{1}\right)_{*}(\mu), \ldots,\left(\Phi_{k}\right)_{*}(\mu)-a_{k}\left(\Psi_{k}\right)_{*}(\mu)\right) .
$$


Clearly, $\tau_{\mathbf{a}}(\mu) \in \mathbb{R}^{k}$, and

$$
\mathbf{a} \in \Omega \Longleftrightarrow \tau_{\mathbf{a}}\left(\mu_{0}\right)=\mathbf{0} \text { for some } \mu_{0} \in \mathcal{M}(X, T) .
$$

Now assume $\mathbf{a} \in \Omega$. Then there exists $\mu_{0} \in \mathcal{M}(X, T)$ such that $\tau_{\mathbf{a}}\left(\mu_{0}\right)=\mathbf{0}$. Apply Theorem 3.1 to obtain that

$$
\begin{aligned}
P_{\mathbf{a}}(\mathbf{q}) & =P\left(T, \sum_{i=1}^{k} q_{i}\left(\Phi_{i}-a_{i} \Psi_{i}\right)\right) \\
& =\sup \left\{h_{\mu}(T)+\mathbf{q} \cdot \tau_{\mathbf{a}}(\mu): \mu \in \mathcal{M}(X, T)\right\} \geq h_{\mu_{0}}(T) \geq 0
\end{aligned}
$$

for each $\mathbf{q}=\left(q_{1}, \ldots, q_{k}\right) \in \mathbb{R}^{k}$.

Conversely, assume $\mathbf{a} \notin \Omega$. write

$$
A=\left\{\tau_{\mathbf{a}}(\mu): \mu \in \mathcal{M}(X, T)\right\} .
$$

By Lemma A.4(i), $\tau_{\mathbf{a}}$ is a continuous affine function on $\mathcal{M}(X, T)$, hence $A$ is a compact convex set in $\mathbb{R}^{k}$. a $\notin \Omega$ implies $\mathbf{0} \notin A$. Hence there exists a unit vector $\mathbf{v} \in \mathbb{R}^{k}$ and $c>0$ such that

$$
\mathbf{v} \cdot \mathbf{b}<-c \quad \text { for any } \mathbf{b} \in A .
$$

By Theorem 3.1, we have for $t>0$,

$$
\begin{aligned}
P_{\mathbf{a}}(t \mathbf{v}) & =\sup \left\{h_{\mu}(T)+t \mathbf{v} \cdot \tau_{\mathbf{a}}(\mu): \mu \in \mathcal{M}(X, T)\right\} \\
& \leq \sup \left\{h_{\mu}(T)-t c: \mu \in \mathcal{M}(X, T)\right\}=h_{\text {top }}(T)-t c .
\end{aligned}
$$

Letting $t \rightarrow+\infty$, we obtain $\inf \left\{P_{\mathbf{a}}(\mathbf{q}): \mathbf{q} \in \mathbb{R}^{k}\right\}=-\infty$. This finishes the proof of (ii).

Next we prove (iii). Fix $\mathbf{a}=\left(a_{1}, \ldots, a_{k}\right) \in \Omega$. Define $A$ as in (5.4). Since $\mathbf{a} \in \Omega$, we have $\mathbf{0} \in A$. Define $g: A \rightarrow \mathbb{R}$ by

$$
g(\mathbf{t})=\sup \left\{h_{\mu}(T): \mu \in \mathcal{M}(X, T), \tau_{\mathbf{a}}(\mu)=\mathbf{t}\right\} .
$$

It is direct to check that $g$ is concave and upper semi-continuous on $A$. By the definition of $H$ (see (5.3)), we have $H(\mathbf{a})=g(\mathbf{0})$. Define

$$
W(\mathbf{q})=\sup \{g(\mathbf{t})+\mathbf{q} \cdot \mathbf{t}: \mathbf{t} \in A\}, \quad \forall \mathbf{q} \in \mathbb{R}^{k} .
$$

Then by Corollary 2.5)(ii), we have $g(\mathbf{t})=\inf \left\{W(\mathbf{q})-\mathbf{q} \cdot \mathbf{t}: \mathbf{q} \in \mathbb{R}^{k}\right\}$ for all $\mathbf{t} \in A$. In particular,

$$
H(\mathbf{a})=g(\mathbf{0})=\inf \left\{W(\mathbf{q}): \mathbf{q} \in \mathbb{R}^{k}\right\} .
$$

However, by Theorem 3.1 and the definition of $W$, we have

$$
W(\mathbf{q})=P\left(T, \sum_{i=1}^{k} q_{i}\left(\Phi_{i}-a_{i} \Psi_{i}\right)\right)=: P_{\mathbf{a}}(\mathbf{q}) .
$$

Hence (5.5) implies $H(\mathbf{a})=\inf _{\mathbf{q} \in \mathbb{R}^{k}} P_{\mathbf{a}}(\mathbf{q})$. This finishes the proof (iii).

In the end we prove (iv). We divide this proof into four steps. 
Step 1. For $\mathbf{a} \in \Omega$, we have $E(\mathbf{a}) \supseteq G_{\mu} \neq \emptyset$ for each $\mu \in \mathcal{M}(X, T)$ with $\left(\Phi_{i}\right)_{*}(\mu)=$ $a_{i}\left(\Psi_{i}\right)_{*}(\mu) \quad(i=1,2, \cdots, k)$. To see this, let $x \in G_{\mu}$. By Lemma A.4(ii) (in which we take $\left.\nu_{n}=\delta_{x}\right)$, we have $\lim _{n \rightarrow \infty}(1 / n) \log \phi_{n, i}(x)=\left(\Phi_{i}\right)_{*}(\mu)$ and $\lim _{n \rightarrow \infty}(1 / n) \log \psi_{n, i}(x)=$ $\left(\Psi_{i}\right)_{*}(\mu)$. It follows that $x \in E(\mathbf{a})$. Hence $E(\mathbf{a}) \supseteq G_{\mu}$.

Step 2. Let $\mathbf{a} \in \mathbb{R}^{k}$ so that $E(\mathbf{a}) \neq \emptyset$. Then for each $x \in E(\mathbf{a})$ and $\mu \in V(x)$ (here $V(x)$ denotes the set of limit points of $\left.\mu_{x, n}=(1 / n) \sum_{j=0}^{n-1} \delta_{T^{j} x}\right)$, we have $\left(\Phi_{i}\right)_{*}(\mu) /\left(\Psi_{i}\right)_{*}(\mu)=a_{i}$ for $i=1,2, \cdots, k$. To show this, take such $x$ and $\mu$. Then there exists a subsequence sequence $n_{\ell}$ of natural numbers such that $\lim _{\ell \rightarrow \infty} \mu_{n_{\ell}, x}=\mu$. By Lemma A.4(ii) again (in which we take $\nu_{n}=\delta_{x}$ ), we have

$$
\lim _{\ell \rightarrow \infty} \frac{1}{n_{\ell}} \log \phi_{n_{\ell}, i}(x)=\left(\Phi_{i}\right)_{*}(\mu) \text { and } \lim _{\ell \rightarrow \infty} \frac{1}{n_{\ell}} \log \psi_{n_{\ell}, i}(x)=\left(\Psi_{i}\right)_{*}(\mu) \quad(i=1,2, \cdots, k) .
$$

Since $x \in E(\mathbf{a})$, we have

$$
\lim _{n \rightarrow \infty} \frac{\log \phi_{n, i}(x)}{\log \psi_{n, i}(x)}=a_{i} \quad(i=1,2, \cdots, k) .
$$

It follows that $\left(\Phi_{i}\right)_{*}(\mu) /\left(\Psi_{i}\right)_{*}(\mu)=a_{i}$ for $i=1,2, \cdots, k$.

Step 3. For $\mathbf{a} \in \Omega$, we have $h_{\mathrm{top}}(T, E(\mathbf{a})) \geq H(\mathbf{a})$. To see it, let $\mu \in \mathcal{M}(X, T)$ so that $\left(\Phi_{i}\right)_{*}(\mu)=a_{i}\left(\Psi_{i}\right) *(\mu)(i=1,2, \cdots, k)$. By step $1, E(\mathbf{a}) \supseteq G_{\mu}$ and hence $h_{\text {top }}(T, E(\mathbf{a})) \geq$ $h_{\mathrm{top}}\left(T, G_{\mu}\right)=h_{\mu}(T)$. This proves the inequality $h_{\mathrm{top}}(T, E(\mathbf{a})) \geq H(\mathbf{a})$.

Step 4. For $\mathbf{a} \in \Omega$, we have $h_{\mathrm{top}}(T, E(\mathbf{a})) \leq H(\mathbf{a})$. By step 2, for each $x \in E(\mathbf{a})$ and $\mu \in V(x)$, we have $\left(\Phi_{i}\right)_{*}(\mu) /\left(\Psi_{i}\right)_{*}(\mu)=a_{i}$ for $i=1,2, \cdots, k$ and hence $h_{\mu}(T) \leq H(\mathbf{a})$. It follows that

$$
E(\mathbf{a}) \subseteq\left\{x \in X: \exists \mu \in V(x) \text { with } h_{\mu}(T) \leq H(\mathbf{a})\right\} .
$$

By Lemma 4.1, we have $h_{\mathrm{top}}(T, E(\mathbf{a})) \leq H(\mathbf{a})$. This finishes the proof of (iv).

We remark that (iii) of Theorem 5.2 can be proved alternatively by applying Proposition 3.15 in $[29$.

Proof of Theorem 1.4. Except the second part in (iii), all the statements listed in Theorem 1.4 follow from Theorem 5.2 (in which we take $k=1$ and $\psi_{n}(x) \equiv 1$ ). In the following, we prove the second part in Theorem 1.4(iii): under the assumptions that $h_{\text {top }}(T)<\infty$ and the entropy map is upper semi-continuous, for any $\alpha \in \Gamma:=\bigcup_{t \in \mathbb{R}}\left\{P_{\Phi}^{\prime}(t-), P_{\Phi}^{\prime}(t+)\right\} \cup$ $\left\{P_{\Phi}^{\prime}( \pm \infty)\right\}$, we have $E_{\Phi}(\alpha) \neq \emptyset$ and

$$
h_{\text {top }}\left(T, E_{\Phi}(\alpha)\right)=\inf \left\{P_{\Phi}(q)-\alpha q: q \in \mathbb{R}\right\} .
$$

According to Corollary 4.5, it suffices to show that if $\alpha \in \Gamma$, then $E_{\Phi}(\alpha) \neq \emptyset$ and $h_{\text {top }}\left(T, E_{\Phi}(\alpha)\right) \geq \inf \left\{P_{\Phi}(q)-\alpha q: q \in \mathbb{R}\right\}$. For this purpose, we will show the following claim: 
Claim. For each $\alpha \in \Gamma$, there exists an ergodic measure $\nu$ such that $\Phi_{*}(\nu)=\alpha$ and $h_{\nu}(T) \geq \inf \left\{P_{\Phi}(q)-\alpha q: q \in \mathbb{R}\right\}$.

The claim will imply that $\nu\left(E_{\Phi}(\alpha)\right)=1$ (by Kingman's sub-additive ergodic theorem), and by Proposition $2.1(3), h_{\text {top }}\left(T, E_{\Phi}(\alpha)\right) \geq h_{\nu}(T) \geq \inf \left\{P_{\Phi}(q)-\alpha q: q \in \mathbb{R}\right\}$. In the following we prove the claim in a way similar to the proof of Theorem 4.8.

First we consider the case $\alpha \in\left\{P_{\Phi}^{\prime}(t-), P_{\Phi}^{\prime}(t+)\right\}$ for some $t \in \mathbb{R}$. Fix $t$ and denote

$$
\mathcal{I}(\Phi, t)=\left\{\mu \in \mathcal{M}(X, T): P_{\Phi}(t)=h_{\mu}(T)+t \Phi_{*}(\mu)\right\} .
$$

By Theorem 3.3(ii), $\mathcal{I}(\Phi, t)$ is a non-empty compact convex subset of $\mathcal{M}(X, T)$. Furthermore, for any $b \in \mathbb{R}$ the set

$$
\mathcal{I}_{b}(\Phi, t):=\left\{\nu \in \mathcal{I}(\Phi, t): \Phi_{*}(\nu)=b\right\}
$$

is compact and convex (may be empty). The convexity of $\mathcal{I}_{b}(\Phi, t)$ is clear. To show the compactness, assume that $\left\{\nu_{n}\right\} \subset \mathcal{I}_{b}(\Phi, t)$ and $\nu_{n}$ converges to $\nu$ in $\mathcal{M}(X, T)$. Then by the upper semi-continuity of $h_{(\cdot)}(T)$ and the continuity of $\Phi_{*}(\cdot)$, we have

$$
h_{\nu}(T)+t \Phi_{*}(\nu) \geq \lim _{n \rightarrow \infty} h_{\nu_{n}}(T)+t \Phi_{*}\left(\nu_{n}\right)=P_{\Phi}(t) .
$$

By Theorem 3.1, $\nu \in \mathcal{I}(\Phi, t)$ and furthermore, $h_{\nu}(T)=h_{\nu_{n}}(T)=P_{\Phi}(t)-t b$ and $\Phi_{*}(\nu)=$ $\Phi_{*}\left(\nu_{n}\right)=b$. This is, $\nu \in \mathcal{I}_{b}(\Phi, t)$. Hence $\mathcal{I}_{b}(\Phi, t)$ is compact.

Since $\alpha \in\left\{P_{\Phi}^{\prime}(t-), P_{\Phi}^{\prime}(t+)\right\} \subset \partial P_{\Phi}(t)$, by Theorem 3.3. $\mathcal{I}_{\alpha}(\Phi, t)$ is non-empty. We are going to show further that $\mathcal{I}_{\alpha}(\Phi, t)$ contains at least one ergodic measure. Since $\mathcal{I}_{\alpha}(\Phi, t)$ is a non-empty compact convex subset of $\mathcal{M}(X, T)$, by the Krein-Milman theorem, it contains at least one extreme point, denoted by $\nu$. Let $\nu=p \nu_{1}+(1-p) \nu_{2}$ for some $0<p<1$ and $\nu_{1}, \nu_{2} \in \mathcal{M}(X, T)$. We will show that $\nu_{1}=\nu_{2}$, which implies that $\nu$ is ergodic. To see that $\nu_{1}=\nu_{2}$, note that

$$
\begin{aligned}
P_{\Phi}(t) & =h_{\nu}(T)+t \Phi_{*}(\nu) \\
& =p\left(h_{\nu_{1}}(T)+t \Phi_{*}\left(\nu_{1}\right)\right)+(1-p)\left(h_{\nu_{2}}(T)+t \Phi_{*}\left(\nu_{2}\right)\right) .
\end{aligned}
$$

By Theorem [3.1, $\nu_{1}, \nu_{2} \in \mathcal{I}(\Phi, t)$. By Theorem 3.3, $\Phi_{*}\left(\nu_{1}\right), \Phi_{*}\left(\nu_{2}\right) \in \partial P_{\Phi}(t)$. Moreover, note that $\alpha=\Phi_{*}(\nu)=p \Phi_{*}\left(\nu_{1}\right)+(1-p) \Phi_{*}\left(\nu_{2}\right)$, we have $\Phi_{*}\left(\nu_{1}\right)=\Phi_{*}\left(\nu_{2}\right)=\alpha$ since $\alpha$ is an extreme point of $\partial P_{\Phi}(t)$ (noting that $\partial P_{\Phi}(t)=\left[P_{\Phi}^{\prime}(t-), P_{\Phi}^{\prime}(t+)\right]$ ). That is, $\nu_{1}, \nu_{2} \in \mathcal{I}_{\alpha}(\Phi, t)$. Since $\nu$ is an extreme point of $\mathcal{I}_{\alpha}(\Phi, t)$, we have $\nu_{1}=\nu_{2}=\nu$. Therefore, $\nu$ is ergodic. Since $\nu \in \mathcal{I}_{\alpha}(\Phi, t)$, we have $\Phi_{*}(\nu)=\alpha$, and $h_{\nu}(T)=P_{\Phi}(t)-\alpha t \geq \inf \left\{P_{\Phi}(q)-\alpha q: q \in \mathbb{R}\right\}$. This proves the claim in the case that $\alpha \in\left\{P_{\Phi}^{\prime}(t-), P_{\Phi}^{\prime}(t+)\right\} \subset \partial P_{\Phi}(t)$ for some $t \in \mathbb{R}$.

Next, we consider the case $\alpha \in\left\{P_{\Phi}^{\prime}( \pm \infty)\right\}$. First assume that $\alpha=P_{\Phi}^{\prime}(+\infty)$. By Theorems 1.1 and 1.2, $\alpha=\bar{\beta}(\Phi)=\max \left\{\Phi_{*}(\mu): \mu \in \mathcal{M}(X, T)\right\}$. By the convexity of $P_{\Phi}(\cdot)$, there exists a sequence $\left(t_{j}\right) \uparrow+\infty$, such that $P_{\Phi}^{\prime}\left(t_{j}\right):=\alpha_{j}$ exists and $\alpha_{j} \uparrow \alpha$ when $j \rightarrow \infty$. As proved in last paragraph, for each $j \in \mathbb{N}$, there exists $\mu_{j} \in \mathcal{M}(X, T)$ such that $\Phi_{*}\left(\mu_{j}\right)=\alpha_{j}$ and $h_{\mu_{j}}(T)=P_{\Phi}\left(t_{j}\right)-\alpha_{j} t_{j}$. Extract a subsequence if necessary so that 
$\lim _{j \rightarrow \infty} \mu_{j}=\mu$ for some $\mu \in \mathcal{M}(X, T)$. Then $\Phi_{*}(\mu)=\lim _{j \rightarrow \infty} \Phi_{*}\left(\mu_{j}\right)=\alpha$ and

$$
\begin{aligned}
h_{\mu}(T) & \geq \limsup _{j \rightarrow \infty} h_{\mu_{j}}(T)=\limsup _{j \rightarrow \infty}\left(P_{\Phi}\left(t_{j}\right)-\alpha_{j} t_{j}\right) \\
& \geq \limsup _{j \rightarrow \infty}\left(P_{\Phi}\left(t_{j}\right)-\alpha t_{j}\right) \geq \inf _{q \in \mathbb{R}}\left\{P_{\Phi}(q)-\alpha q\right\},
\end{aligned}
$$

where we use the facts $\alpha_{j} \leq \alpha$ and $t_{j}>0$ for the second inequality. Now let $\mu=\int \theta d m(\theta)$ be the ergodic decomposition of $\mu \in \mathcal{M}(X, T)$. By Proposition A.1 (3), $\int \Phi_{*}(\theta) d m(\theta)=$ $\Phi_{*}(\mu)=\alpha$. By the way, we also have $\int h_{\theta}(T) d m(\theta)=h_{\mu}(T)$ (cf. [49]). Note that $\Phi_{*}(\theta) \leq \alpha$ for any $\theta$. Hence there exists an ergodic measure $\nu$ such that $\Phi_{*}(\nu)=\alpha$ and $h_{\nu}(T) \geq h_{\mu}(T) \geq \inf _{q \in \mathbb{R}}\left\{P_{\Phi}(q)-\alpha q\right\}$, as desired. In the end, assume $\alpha=P_{\Phi}^{\prime}(-\infty)$. Then $-\alpha=P_{-\Phi}^{\prime}(+\infty)$. Since $-\Phi$ is also asymptotically additive, there exists an ergodic measure $\eta$ such that $(-\Phi)_{*}(\eta)=-\alpha$, i.e., $\Phi_{*}(\eta)=\alpha$, and

$$
h_{\eta}(T) \geq \inf _{q \in \mathbb{R}}\left\{P_{-\Phi}(q)-(-\alpha) q\right\}=\inf _{q \in \mathbb{R}}\left\{P_{\Phi}(-q)+\alpha q\right\}=\inf _{q \in \mathbb{R}}\left\{P_{\Phi}(q)-\alpha q\right\} .
$$

This finishes the proof of the claim, and also the proof of Theorem 1.4.

\section{EXAmples}

In this section, we give some examples regarding Lyapunov spectra on TDS's on which the entropy map is not upper semi-continuous. The multifractal behaviors in this case are rather irregular and complicated. These examples also show that the conditions and results in Theorems 1.1, 1.2 and 4.2 are optimal.

Example 6.1. There exist a TDS $(X, T)$ with $h_{\mathrm{top}}(T)<\infty$ and an additive potential $\Phi=\left\{\log \phi_{n}\right\}_{n=1}^{\infty}$ on $X$ such that $\left(\lim _{t \rightarrow 0+} P_{\Phi}^{\prime}(t-), P_{\Phi}^{\prime}(\infty)\right)=\emptyset$ and for $\alpha=P_{\Phi}^{\prime}(\infty)$,

$$
h_{\text {top }}\left(T, E_{\Phi}(\alpha)\right)=\sup \left\{h_{\mu}(T): \mu \in \mathcal{M}(X, T), \Phi_{*}(\mu)=\alpha\right\}<\inf _{q>0}\left\{P_{\Phi}(q)-\alpha q\right\} .
$$

Construction. According to Krieger [36, for each $i \in \mathbb{N}$, we can construct a Cantor set $X_{i} \subseteq\left[0, \frac{1}{i}\right] \times\left\{\frac{1}{i}\right\}$ and a continuous transformation $T_{i}: X_{i} \rightarrow X_{i}$ such that $\left(X_{i}, T_{i}\right)$ is uniquely ergodic (i.e., $\mathcal{M}\left(X_{i}, T_{i}\right)$ consists of a singleton) and $h_{\text {top }}\left(T_{i}\right)=1$. Then we let $X=\bigcup_{i=1}^{\infty} X_{i} \cup\{(0,0)\}$ and define $T: X \rightarrow X$ by

$$
T(x)= \begin{cases}T_{i}(x) & \text { if } x \in X_{i} \\ x & \text { if } x=(0,0)\end{cases}
$$

It is easy to check that $(X, T)$ is a TDS. Define a function $g: X \rightarrow \mathbb{R}$ by

$$
g(x)= \begin{cases}1-1 / i & \text { if } x \in X_{i} \\ 1 & \text { if } x=(0,0)\end{cases}
$$

Let $\phi_{n}(x)=\exp \left(\sum_{j=0}^{n-1} g\left(T^{j} x\right)\right)$ for $n \in \mathbb{N}$ and $x \in X$. Then $\Phi=\left\{\log \phi_{n}\right\}_{n=1}^{\infty}$ is an additive potential on $X$. 
For $i \in \mathbb{N}$, let $\mu_{i}$ denote the unique element in $\mathcal{M}\left(X_{i}, T_{i}\right)$. Let $\mu_{0}$ be the Dirac measure $\delta_{(0,0)}$ at the point $(0,0)$. Then $\mathcal{E}(X, T)=\left\{\mu_{i}: i=0,1, \cdots\right\}$ and thus

$$
\mathcal{M}(X, T)=\left\{\sum_{i=0}^{\infty} \lambda_{i} \mu_{i}: \lambda_{i} \geq 0 \text { and } \sum_{i=0}^{\infty} \lambda_{i}=1\right\} .
$$

By Theorem 3.1, we have

$$
\begin{aligned}
P_{\Phi}(q) & =\sup \left\{h_{\mu}(T)+q \Phi_{*}(\mu): \mu \in \mathcal{M}(X, T)\right\} \\
& =\sup \left\{h_{\sum_{i=0}^{\infty} \lambda_{i} \mu_{i}}(T)+q \sum_{i=0}^{\infty} \lambda_{i} \Phi_{*}\left(\mu_{i}\right): \lambda_{i} \geq 0 \text { and } \sum_{i=0}^{\infty} \lambda_{i}=1\right\} \\
& =\sup \left\{\sum_{i=0}^{\infty} \lambda_{i}\left(h_{\mu_{i}}(T)+q \Phi_{*}\left(\mu_{i}\right)\right): \lambda_{i} \geq 0 \text { and } \sum_{i=0}^{\infty} \lambda_{i}=1\right\} \\
& \left.=\sup \left\{q \lambda_{0}+\sum_{i=1}^{\infty} \lambda_{i}(1+q(1-1 / i))\right): \lambda_{i} \geq 0 \text { and } \sum_{i=0}^{\infty} \lambda_{i}=1\right\} \\
& =\max \left\{q, \sup _{i \in \mathbb{N}}\{1+q(1-1 / i)\}\right\}=q+1 \text { for } q>0 .
\end{aligned}
$$

Hence $P_{\Phi}^{\prime}(q)=1$ for $q>0$ and $P_{\Phi}^{\prime}(\infty)=1$. Thus $\left(\lim _{t \rightarrow 0+} P_{\Phi}^{\prime}(t-), P_{\Phi}^{\prime}(\infty)\right)=\emptyset$. For $\alpha=P_{\Phi}^{\prime}(\infty)=1$, one has $E_{\Phi}(\alpha)=\{(0,0)\}$. Hence

$$
0=h_{\mathrm{top}}\left(T, E_{\Phi}(\alpha)\right)=\sup \left\{h_{\mu}(T): \mu \in \mathcal{M}(X, T), \Phi_{*}(\mu)=\alpha\right\}<\inf _{q>0}\left\{P_{\Phi}(q)-\alpha q\right\}=1,
$$

as desired.

Example 6.2. There exist a $T D S(X, T)$ with $h_{\mathrm{top}}(T)<\infty$, an additive potential $\Phi=$ $\left\{\log \phi_{n}\right\}_{n=1}^{\infty}$ on $X$ such that for each $\alpha \in[\underline{\beta}(\Phi), \bar{\beta}(\Phi)]$,

$$
h_{\text {top }}\left(T, E_{\Phi}(\alpha)\right)<\inf _{q \in \mathbb{R}}\left\{P_{\Phi}(q)-\alpha q\right\}
$$

where $\underline{\beta}(\Phi):=\lim _{n \rightarrow \infty} \frac{1}{n} \inf _{x \in X} \log \phi_{n}(x)$.

Construction. Similar to the construction in Example 6.1, we construct Cantor sets $X_{i} \subseteq$ $\left[0, \frac{1}{|i|+1}\right] \times\left\{\frac{i}{|i|+1}\right\}(i \in \mathbb{Z})$ and continuous transformations $T_{i}: X_{i} \rightarrow X_{i}$ such that $\left(X_{i}, T_{i}\right)$ is uniquely ergodic and $h_{\text {top }}\left(T_{i}\right)=\frac{|i|}{|i|+1}$. Then let $X=\bigcup_{i \in \mathbb{Z}} X_{i} \cup\{(0,1)\} \cup\{(0,-1)\}$ and define $T: X \rightarrow X$ by

$$
T(x)= \begin{cases}T_{i}(x) & \text { if } x \in X_{i} \\ x & \text { if } x=(0,1) \text { or }(0,-1) .\end{cases}
$$

It is clear that $(X, T)$ is a TDS. Define a continuous function $h$ on $X$ by

$$
g(x)= \begin{cases}\frac{i}{|i|+1} & \text { if } x \in X_{i}, \\ 1 & \text { if } x=(0,1) \\ -1 & \text { if } x=(0,-1)\end{cases}
$$


Let $\phi_{n}(x)=\exp \left(\sum_{j=0}^{n-1} g\left(T^{j} x\right)\right)$ for $n \in \mathbb{N}$ and $x \in X$. Then $\Phi=\left\{\log \phi_{n}\right\}$ is an additive potential on $X$ with $[\underline{\beta}(\Phi), \bar{\beta}(\Phi)]=[-1,1]$. Similarly, it is not hard to verify that

$$
P_{\Phi}(q)=\max \left\{q,-q, \sup _{i \in \mathbb{Z}}\left\{\frac{|i|}{|i|+1}+\frac{i}{|i|+1} q\right\}\right\}=1+|q| .
$$

Hence $P_{\Phi}^{\prime}(\infty)=P_{\Phi}^{\prime}(0+)=1, P_{\Phi}^{\prime}(0-)=P_{\Phi}^{\prime}(-\infty)=-1$ and

$$
P_{\Phi}^{\prime}(q)= \begin{cases}1 & \text { if } q>0 \\ -1 & \text { if } q<0\end{cases}
$$

It is easy to see that $E_{\Phi}(\alpha) \neq \emptyset$ if and only if $\alpha \in\left\{\frac{i}{|i|+1}: i \in \mathbb{Z}\right\} \cup\{1,-1\}$. Furthermore

$$
E_{\Phi}(\alpha)= \begin{cases}X_{i} & \text { if } \alpha=\frac{i}{|i|+1} \text { for some } i \in \mathbb{Z} \\ \{(0,1)\} & \text { if } \alpha=1, \\ \{(0,-1)\} & \text { if } \alpha=-1\end{cases}
$$

Hence for $\alpha \in[\underline{\beta}(\Phi), \bar{\beta}(\Phi)]=[-1,1]$,

$$
h_{\text {top }}\left(T, E_{\Phi}(\alpha)\right)<1=\inf _{q \in \mathbb{R}}\left\{P_{\Phi}(q)-\alpha q\right\},
$$

as desired. Keep in mind that $\left\{\Phi_{*}(\mu): \mu \in \mathcal{M}(X, T)\right\}=[-1,1]$ by Lemma A.3.

Example 6.3. There exist a TDS $(X, T)$ with $h_{\mathrm{top}}(T)<\infty$ and two additive potential $\Phi_{i}=\left\{\log \phi_{n, i}\right\}_{n=1}^{\infty}(i=1,2)$ on $X$ such that $\partial P_{\boldsymbol{\Phi}}\left(\mathbb{R}_{+}^{2}\right)$ is one-dimensional set and for any $\mathbf{a} \in \partial P_{\boldsymbol{\Phi}}\left(\mathbb{R}_{+}^{2}\right)$, where $\mathbf{\Phi}=\left(\Phi_{1}, \Phi_{2}\right)$,

$$
\sup \left\{h_{\mu}(T): \mu \in \mathcal{M}(X, T), \boldsymbol{\Phi}_{*}(\mu)=\mathbf{a}\right\}<\inf _{\mathbf{q} \in \mathbb{R}_{+}^{2}}\left\{P_{\boldsymbol{\Phi}}(\mathbf{q})-\mathbf{a} \cdot \mathbf{q}\right\} .
$$

Construction. Similar to the previous two examples, we construct a Cantor set $X_{i} \subseteq$ $\left[0, \frac{1}{|i|+1}\right] \times\left\{\frac{i}{|i|+1}\right\}$ and a continuous transformation $T_{i}: X_{i} \rightarrow X_{i}$ such that $\left(X_{i}, T_{i}\right)$ is uniquely ergodic and $h_{\text {top }}\left(T_{i}\right)=1$. Then let $X=\bigcup_{i \in \mathbb{Z}} X_{i} \cup\{(0,1)\} \cup\{(0,-1)\}$ and define $T: X \rightarrow X$ by

$$
T(x)= \begin{cases}T_{i}(x) & \text { if } x \in X_{i} \\ x & \text { if } x=(0,1) \text { or }(0,-1)\end{cases}
$$

It is clear that $(X, T)$ is a TDS. Define two continuous function $g_{1}, g_{2}$ on $X$ by

$$
g_{1}(x)=\left\{\begin{array}{ll}
\frac{i}{i+1} & \text { if } x \in X_{i}, i \geq 0, \\
1 & \text { if } x=(0,1), \\
\frac{2|i|}{|i|+1} & \text { if } x \in X_{i}, i<0, \\
2 & \text { if } x=(0,-1) .
\end{array} \quad \text { and } \quad g_{2}(x)= \begin{cases}0 & \text { if } x \in X_{i}, i \geq 0 \\
0 & \text { if } x=(0,1), \\
\frac{-|i|}{|i|+1} & \text { if } x \in X_{i}, i<0 \\
-1 & \text { if } x=(0,-1) .\end{cases}\right.
$$

Set $\phi_{n, i}(x)=\exp \left(\sum_{j=0}^{n-1} g_{i}\left(T^{j} x\right)\right)$ for $i=1,2, n \in \mathbb{N}$ and $x \in X$. Then $\Phi_{i}=\left\{\log \phi_{n, i}\right\}_{n=1}^{\infty}$, $i=1,2$, are two additive potentials on $X$ with $\bar{\beta}\left(\Phi_{1}\right)=2, \bar{\beta}\left(\Phi_{2}\right)=0$. 
For $i \in \mathbb{Z}$, let $\mu_{i}$ denote the unique element in $\mathcal{M}\left(X_{i}, T_{i}\right)$. Let $\mu_{\infty}$ be the Dirac measure $\delta_{(0,1)}$ at the point $(0,1)$. Let $\mu_{-\infty}$ be the Dirac measure $\delta_{(0,-1)}$ at the point $(0,-1)$. For simplify, write $\overline{\mathbb{Z}}=\mathbb{Z} \cup\{ \pm \infty\}$. Then $\mathcal{E}(X, T)=\left\{\mu_{i}: i \in \overline{\mathbb{Z}}\right\}$. A direct calculation by applying Theorem 3.1 yields that for $\mathbf{q}=\left(q_{1}, q_{2}\right) \in \mathbb{R}_{+}^{2}$,

$$
P_{\mathbf{\Phi}}(\mathbf{q})=\max \left\{1+q_{1}, 1+2 q_{1}-q_{2}\right\} .
$$

Hence

$$
P_{\mathbf{\Phi}}^{\prime}(\mathbf{q})= \begin{cases}(1,0) & \text { if } \mathbf{q} \in \mathbb{R}_{+}^{2} \text { with } q_{1}<q_{2} \\ (2,-1) & \text { if } \mathbf{q} \in \mathbb{R}_{+}^{2} \text { with } q_{1}>q_{2}\end{cases}
$$

and $\partial P_{\boldsymbol{\Phi}}((q, q))=\operatorname{conv}(\{(1,0),(2,-1)\})$ for $q>0$. Thus $\partial P_{\boldsymbol{\Phi}}\left(\mathbb{R}_{+}^{2}\right)=\operatorname{conv}(\{(1,0),(2,-1)\})$ is one dimensional.

Recall that $A:=\left\{\boldsymbol{\Phi}_{*}(\mu): \mu \in \mathcal{M}(X, T)\right\}$. Clearly, $A=\operatorname{conv}(\{(0,0),(1,0),(2,-1)\})$ is a two-dimensional set and $\partial P_{\boldsymbol{\Phi}}\left(\mathbb{R}_{+}^{2}\right)$ is just one edge in the convex set $A$.

For $\mathbf{a} \in \partial P_{\boldsymbol{\Phi}}\left(\mathbb{R}_{+}^{2}\right)$, there exists unique $t \in[0,1]$ with $\mathbf{a}=t(1,0)+(1-t)(2,-1)$. It is not hard to see that for $\mu \in \mathcal{M}(X, T), \mathbf{\Phi}_{*}(\mu)=\mathbf{a}$ if and only if $\mu=t \mu_{\infty}+(1-t) \mu_{-\infty}$. Hence

$$
\begin{aligned}
\sup & \left\{h_{\mu}(T): \mu \in \mathcal{M}(X, T), \boldsymbol{\Phi}_{*}(\mu)=\mathbf{a}\right\}=h_{t \mu_{\infty}+(1-t) \mu_{-\infty}}(T)=0 \\
& <1=\inf _{\mathbf{q} \in \mathbb{R}_{+}^{2}}\left\{\max \left\{1+q_{1}, 1+2 q_{1}-q_{2}\right\}-\left(t q_{1}+(1-t)\left(2 q_{1}-q_{2}\right)\right)\right\} \\
& =\inf _{\mathbf{q} \in \mathbb{R}_{+}^{2}}\left\{P_{\mathbf{\Phi}}(\mathbf{q})-\mathbf{a} \cdot \mathbf{q}\right\},
\end{aligned}
$$

as desired.

\section{Appendix A. Properties and examples of Asymptotical sub-Additive POTENTIALS}

In this appendix, we give some properties and examples of asymptotically sub-additive (resp. asymptotically additive) potentials. Let $(X, T)$ be a TDS and let $\Phi=\left\{\log \phi_{n}\right\}_{n=1}^{\infty}$ be an asymptotically sub-additive potential on a TDS $(X, T)$. Let $\lambda_{\Phi}$ and $\Phi_{*}$ be defined as in (1.2)-(1.3).

Proposition A.1. Let $\mu \in \mathcal{M}(X, T)$. Then we have the following properties.

(1) The limit $\Phi_{*}(\mu)=\lim _{n \rightarrow \infty} \frac{1}{n} \int \log \phi_{n}(x) d \mu(x)$ exists (which may take value $\left.-\infty\right)$. Furthermore $\lambda_{\Phi}(x)$ exists for $\mu$-a.e. $x \in X$, and $\int \lambda_{\Phi}(x) d \mu(x)=\Phi_{*}(\mu)$. In particular, when $\mu \in \mathcal{E}(X, T), \lambda_{\Phi}(x)=\Phi_{*}(\mu)$ for $\mu$-a.e. $x \in X$.

(2) The map $\Phi_{*}: \mathcal{M}(X, T) \rightarrow \mathbb{R} \cup\{-\infty\}$ is upper semi-continuous and there is $C \in \mathbb{R}$ such that for all $\mu \in \mathcal{M}(X, T), \lambda_{\Phi}(x) \leq C \mu$-a.e and $\Phi_{*}(\mu) \leq C$.

(3) Let $\mu=\int_{\Omega} \theta d m(\theta)$ be the ergodic decomposition of $\mu \in \mathcal{M}(X, T)$. Then $\Phi_{*}(\mu)=$ $\int_{\Omega} \Phi_{*}(\theta) d m(\theta)$. 
Proof. In the case that $\Phi$ is sub-additive, statement (1) comes exactly from Kingman's sub-additive ergodic theorem (cf. [49, p. 231). We shall show that it remains valid when $\Phi$ is asymptotically sub-additive. Fix such a $\Phi$. For $\epsilon>0$, by definition, there exist a subadditive potential $\Psi=\left\{\log \psi_{n}\right\}_{n=1}^{\infty}$ and an integer $n_{0}$ such that $\left|\log \phi_{n}(x)-\log \psi_{n}(x)\right| \leq n \epsilon$ for any $n \geq n_{0}$ and $x \in X$. Hence

$$
\begin{aligned}
\limsup _{n \rightarrow \infty} \frac{1}{n} \int \log \phi_{n}(x) d \mu(x) & \leq \lim _{n \rightarrow \infty} \frac{1}{n} \int \log \psi_{n}(x) d \mu(x)+\epsilon \\
& \leq \liminf _{n \rightarrow \infty} \frac{1}{n} \int \log \phi_{n}(x) d \mu(x)+2 \epsilon .
\end{aligned}
$$

Since the above inequalities hold for any $\epsilon>0$, the limit for defining $\Phi_{*}(\mu)$ exists. Similarly, we have the inequalities

$$
\limsup _{n \rightarrow \infty} \frac{1}{n} \log \phi_{n}(x) \leq \lim _{n \rightarrow \infty} \frac{1}{n} \log \psi_{n}(x)+\epsilon \leq \liminf _{n \rightarrow \infty} \frac{1}{n} \log \phi_{n}(x)+2 \epsilon
$$

for $\mu$-a.e. $x$, from which we derive that $\lambda_{\Phi}(x)$ exists $\mu$-a.e and $\int \lambda_{\Phi}(x) d \mu(x)=\Phi_{*}(\mu)$. Furthermore, $\lambda_{\Phi}(x)=\Phi_{*}(\mu) \mu$-a.e. when $\mu$ is ergodic. This proves (1).

To see that $\Phi_{*}$ is upper semi-continuous, let $\epsilon>0$ and $\Psi$ be given as in the above paragraph. Suppose that $\left\{\mu_{i}\right\}$ is a sequence in $\mathcal{M}(X, T)$ which converges to $\mu$ in the weak* topology. Then for any $n \geq n_{0}$ and $R \in \mathbb{R}$,

$$
\begin{aligned}
\limsup _{i \rightarrow \infty} \Phi_{*}\left(\mu_{i}\right) & \leq \limsup _{i \rightarrow \infty} \Psi_{*}\left(\mu_{i}\right)+\epsilon \leq \limsup _{i \rightarrow \infty} \frac{1}{n} \int \log \psi_{n}(x) d \mu_{i}(x)+\epsilon \\
& \leq \limsup _{i \rightarrow \infty} \frac{1}{n} \int \max \left\{\log \psi_{n}(x), R\right\} d \mu_{i}(x)+\epsilon \\
& =\frac{1}{n} \int \max \left\{\log \psi_{n}(x), R\right\} d \mu(x)+\epsilon .
\end{aligned}
$$

Taking $R \rightarrow-\infty$ to obtain

$$
\limsup _{i \rightarrow \infty} \Phi_{*}\left(\mu_{i}\right) \leq \frac{1}{n} \int \log \psi_{n}(x) d \mu(x)+\epsilon \leq \frac{1}{n} \int \log \phi_{n}(x) d \mu(x)+2 \epsilon .
$$

Letting $n \rightarrow \infty$, we have $\lim \sup _{i \rightarrow \infty} \Phi_{*}\left(\mu_{i}\right) \leq \Phi_{*}(\mu)$. This proves the upper semi-continuity of $\Phi_{*}$. To give an upper bound for $\lambda_{\Phi}$ and $\Phi_{*}$, let $D=\max _{x \in X} \psi_{n_{0}}(x)$. Then $\log \psi_{k n_{0}}(x) \leq$ $k \log D$ by the subadditivity. Hence for $\mu$-a.e $x$,

$$
\lambda_{\Phi}(x) \leq \epsilon+\limsup _{k \rightarrow \infty} \frac{1}{k n_{0}} \log \psi_{k n_{0}}(x) \leq \epsilon+(\log D) / n_{0} .
$$

Take integration with respect to $\mu$ to get $\Phi_{*}(\mu) \leq \epsilon+(\log D) / n_{0}$.

To prove (3), we first assume that $\Phi$ is sub-additive. Let $\mu=\int_{\Omega} \theta d m(\theta)$ be the ergodic decomposition of $\mu \in \mathcal{M}(X, T)$. Let $C_{1}=\max _{x \in X}\left|\log \phi_{1}(x)\right|$. Then

$$
\frac{1}{n} \int \log \phi_{n}(x) d \theta(x) \leq C_{1} \quad \text { for all } \theta \in \Omega, n \in \mathbb{N} .
$$


Define $h_{k}(\theta)=\frac{1}{2^{k}} \int \log \phi_{2^{k}}(x) d \theta(x)$ for $\theta \in \mathcal{M}(X, T)$ and $k \in \mathbb{N}$. Since $\Phi$ is sub-additive and $\theta$ is invariant, we have $C_{1} \geq h_{1}(\theta) \geq h_{2}(\theta) \geq \cdots$ and $h_{k}(\theta) \searrow \Phi_{*}(\theta)$. By (A.1), we have

$$
\begin{aligned}
\Phi_{*}(\mu) & =\lim _{n \rightarrow \infty} \frac{1}{n} \int \log \phi_{n}(x) d \mu(x)=\lim _{k \rightarrow \infty} \int_{\Omega} \frac{1}{2^{k}} \int \log \phi_{2^{k}}(x) d \theta(x) d m(\theta) \\
& =\lim _{k \rightarrow \infty} \int h_{k}(\theta) d m(\theta)=\int_{\Omega} \lim _{k \rightarrow \infty} h_{k}(\theta) d m(\theta)=\int_{\Omega} \Phi_{*}(\theta) d m(\theta),
\end{aligned}
$$

where we use the monotone convergence theorem for the fourth equality. Hence we prove (3) in the case that $\Phi$ is sub-additive. Now assume that $\Phi$ is asymptotically sub-additive. For $\epsilon>0$, let $\Psi$ be given as in the first paragraph of our proof. Then $\left|\Phi_{*}(\theta)-\Psi_{*}(\theta)\right| \leq \epsilon$ for any $\theta \in \mathcal{M}(X, T)$. It together with $\Psi_{*}(\mu)=\int_{\Omega} \Psi_{*}(\theta) d m(\theta)$ yields $\left|\Phi_{*}(\mu)-\int_{\Omega} \Phi_{*}(\theta) d m(\theta)\right| \leq$ $2 \epsilon$. Letting $\epsilon \rightarrow 0$, we obtain the desired identity for $\Phi$. This finishes the proof.

Let $\mathcal{M}(X)$ denote the space of Borel probability measures on $X$ endowed with the weakstar topology. Then we have

Lemma A.2. Suppose $\left\{\nu_{n}\right\}_{n=1}^{\infty}$ is a sequence in $\mathcal{M}(X)$. We form the new sequence $\left\{\mu_{n}\right\}_{n=1}^{\infty}$ by $\mu_{n}=\frac{1}{n} \sum_{i=0}^{n-1} \nu_{n} \circ T^{-i}$. Assume that $\mu_{n_{i}}$ converges to $\mu$ in $\mathcal{M}(X)$ for some subsequence $\left\{n_{i}\right\}$ of natural numbers. Then $\mu \in \mathcal{M}(X, T)$ and

$$
\limsup _{i \rightarrow \infty} \frac{1}{n_{i}} \int \log \phi_{n_{i}}(x) d \nu_{n_{i}}(x) \leq \Phi_{*}(\mu) .
$$

Proof. The lemma was proved in [15, Lemma 2.3] for the case that $\Phi$ is sub-additive. Here we shall show that it can be extended to the case that $\Phi$ is asymptotically sub-additive.

Let $\Phi$ be an asymptotically sub-additive potential on $X$ and $\epsilon>0$. Then there exist a sub-additive potential $\Psi=\left\{\log \psi_{n}\right\}_{n=1}^{\infty}$ on $X$ and $n_{0}$ such that $\left|\log \phi_{n}(x)-\log \psi_{n}(x)\right| \leq n \epsilon$ for any $n \geq n_{0}$ and $x \in X$. Hence

$\limsup _{i \rightarrow \infty} \frac{1}{n_{i}} \int \log \phi_{n_{i}}(x) d \nu_{n_{i}}(x) \leq \limsup _{i \rightarrow \infty} \frac{1}{n_{i}} \int \log \psi_{n_{i}}(x) d \nu_{n_{i}}(x)+\epsilon \leq \Psi_{*}(\mu)+\epsilon \leq \Phi_{*}(\mu)+2 \epsilon$.

Letting $\epsilon \rightarrow 0$, we obtain the desired inequality for $\Phi$.

Lemma A.3. Define $\bar{\beta}(\Phi)=\lim \sup _{n \rightarrow \infty} \sup _{x \in X} \frac{\log \phi_{n}(x)}{n}$. Then

(1) $\bar{\beta}(\Phi) \in \mathbb{R} \cup\{-\infty\}$ and $\bar{\beta}(\Phi)=\liminf _{n \rightarrow \infty} \sup _{x \in X} \frac{\log \phi_{n}(x)}{n}$.

(2) $\bar{\beta}(\Phi)=\sup \left\{\Phi_{*}(\mu): \mu \in \mathcal{M}(X, T)\right\}$ and there exists an ergodic measure $\nu \in$ $\mathcal{M}(X, T)$ such that $\bar{\beta}(\Phi)=\Phi_{*}(\nu)$.

(3) The following conditions are equivalent:

(a) $\bar{\beta}(\Phi)=-\infty$;

(b) $\lambda_{\Phi}(x)=-\infty$ for all $x \in X$;

(c) $\Phi_{*}(\mu)=-\infty$ for all $\mu \in \mathcal{M}(X, T)$;

(d) $P(T, \Phi)=-\infty$. 
(4) If $\bar{\beta}(\Phi)>-\infty$, then $h_{\mathrm{top}}(T)+\bar{\beta}(\Phi) \geq P(T, \Phi) \geq \bar{\beta}(\Phi)>-\infty$. Moreover if we assume in addition that $h_{\mathrm{top}}(T)<\infty$, then $P(T, \Phi) \in \mathbb{R}$.

Proof. Let $\epsilon>0$. Take a sub-additive potential $\Psi=\left\{\log \psi_{n}\right\}_{n=1}^{\infty}$ on $(X, T)$ such that $\left|\log \phi_{n}(x)-\log \psi_{n}(x)\right|<n \epsilon$ for all $n \geq n_{0}$ and $x \in X$. Let $C=\max _{x \in X}\left|\psi_{1}(x)\right|$. Then $\psi_{n}(x) \leq C^{n}$. Thus for $n \geq n_{0}$ we have $\log \phi_{n}(x) \leq n(\log C+\epsilon)$ and hence $\sup _{x \in X} \frac{\log \phi_{n}(x)}{n} \leq$ $\log C+\epsilon$. This implies $\bar{\beta}(\Phi) \in \mathbb{R} \cup\{-\infty\}$. Denote $b_{n}=\sup _{x \in X} \log \psi_{n}(x)$. Then by the sub-additivity of $\Psi, b_{n+m} \leq b_{n}+b_{m}$. It follows that $\liminf _{n \rightarrow \infty} b_{n} / n=\limsup _{n \rightarrow \infty} b_{n} / n$ and thus $\liminf \operatorname{in}_{n \rightarrow \infty} \sup _{x \in X} \log \phi_{n}(x) / n \geq \lim \sup _{n \rightarrow \infty} \sup _{x \in X} \log \phi_{n}(x) / n-2 \epsilon$. Letting $\epsilon \rightarrow 0$, we obtain

$$
\liminf _{n \rightarrow \infty} \sup _{x \in X} \log \phi_{n}(x) / n=\limsup _{n \rightarrow \infty} \sup _{x \in X} \log \phi_{n}(x) / n .
$$

This proves (1).

For any $\mu \in \mathcal{M}(X, T)$, by Proposition A.1(1),

$$
\Phi_{*}(\mu)=\lim _{n \rightarrow \infty} \int_{X} \frac{\log \phi_{n}(x)}{n} d \mu(x) \leq \limsup _{n \rightarrow \infty} \sup _{x \in X} \frac{\log \phi_{n}(x)}{n}=\bar{\beta}(\Phi) .
$$

Hence $\sup \left\{\Phi_{*}(\mu): \mu \in \mathcal{M}(X, T)\right\} \leq \bar{\beta}(\Phi)$. Conversely, choose $n_{i} \rightarrow \infty$ and $x_{i} \in X$ such that $\lim _{i \rightarrow \infty} \frac{\log \phi_{n_{i}}\left(x_{i}\right)}{n_{i}}=\lim \sup _{n \rightarrow \infty} \sup _{x \in X} \frac{\log \phi_{n}(x)}{n}$. Let $\mu_{n_{i}}=\frac{1}{n_{i}} \sum_{j=0}^{n_{i}-1} \delta_{T^{j} x_{i}}$ for $i \in \mathbb{N}$. Since $\mathcal{M}(X)$ is compact, we may assume that $\mu_{n_{i}} \rightarrow \mu$ for some $\mu \in \mathcal{M}(X)$. By Lemma A.2. $\mu \in \mathcal{M}(X, T)$ and $\lim _{i \rightarrow \infty} \frac{1}{n_{i}} \int \log \phi_{n_{i}}(x) d \delta_{x_{i}} \leq \Phi_{*}(\mu)$, i.e., $\bar{\beta}(\Phi)=\lim _{i \rightarrow \infty} \frac{\log \phi_{n_{i}}\left(x_{i}\right)}{n_{i}} \leq$ $\Phi_{*}(\mu)$. Moreover, by Proposition A.1 (3), there exists an ergodic measure $\nu \in \mathcal{M}(X, T)$ such that $\bar{\beta}(\Phi) \leq \Phi_{*}(\nu)$. Clearly, $\bar{\beta}(\Phi)=\Phi_{*}(\nu)$. This proves $(2)$.

To show (3), note that the implications $(a) \Rightarrow(b),(c)$ are direct. By (2), there exists an ergodic measure $\nu \in \mathcal{M}(X, T)$ such that $\bar{\beta}(\Phi)=\Phi_{*}(\nu)$. By Proposition A.1 $(1), \lambda_{\Phi}(x)=$ $\bar{\beta}(\Phi)$ for $\nu$-a.e. $x \in X$. Hence $\bar{\beta}(\Phi)=-\infty$ when $(b)$ or $(c)$ occurs. This shows that (b) or (c) implies $(a)$. The equivalence of $(c)$ and $(d)$ comes from Theorem 3.1, This proves (3). Part (4) follows directly from (2) and Theorem 3.1.

Now we give some properties of asymptotically additive potentials, which just follow from Proposition A.1(2) and Lemma A.2.

Lemma A.4. Assume that $\Phi=\left\{\log \phi_{n}\right\}_{n=1}^{\infty}$ is an asymptotically additive potential on $(X, T)$. Then

(i) The map $\mu \mapsto \Phi_{*}(\mu)$ is continuous on $\mathcal{M}(X, T)$.

(ii) Suppose $\left\{\nu_{n}\right\}_{n=1}^{\infty}$ is a sequence in $\mathcal{M}(X)$. We form the new sequence $\left\{\mu_{n}\right\}_{n=1}^{\infty}$ by $\mu_{n}=\frac{1}{n} \sum_{i=0}^{n-1} \nu_{n} \circ T^{-i}$. Assume that $\mu_{n_{i}}$ converges to $\mu$ in $\mathcal{M}(X)$ for some subsequence $\left\{n_{i}\right\}$ of natural numbers. Then $\mu \in \mathcal{M}(X, T)$, and moreover

$$
\lim _{i \rightarrow \infty} \frac{1}{n_{i}} \int \log \phi_{n_{i}}(x) d \nu_{n_{i}}(x)=\Phi_{*}(\mu) .
$$


(iii) $\Omega:=\left\{\Phi_{*}(\mu): \mu \in \mathcal{M}(X, T)\right\}$ is an interval which equals $[\underline{\beta}(\Phi), \bar{\beta}(\Phi)]$, where $\underline{\beta}(\Phi):=\lim _{n \rightarrow \infty}(1 / n) \inf _{x \in X} \log \phi_{n}(x)$.

In the end of this section, we give the following proposition.

Proposition A.5. Let $\Phi=\left\{\log \phi_{n}\right\}$ be a potential on $X$ (i.e., each $\phi_{n}$ is a non-negative continuous function on $X)$. We have the following statements.

(i) If there exists $C \geq 1$ such that $\phi_{n+m}(x) \leq C \phi_{n}(x) \phi_{m}\left(T^{n} x\right)$ for all $x \in X$ and $n, m \in \mathbb{N}$, then $\Phi$ is asymptotically sub-additive.

(ii) If there exists $C \geq 1$ such that

$$
0<C^{-1} \phi_{n}(x) \phi_{m}\left(T^{n} x\right) \leq \phi_{n+m}(x) \leq C \phi_{n}(x) \phi_{m}\left(T^{n} x\right)
$$

for all $x \in X$ and $n, m \in \mathbb{N}$, then $\Phi$ is asymptotically additive.

(iii) If $\phi_{n}(x)>0$ for all $n \in \mathbb{N}, x \in X$ and there exists a continuous function $g$ on $X$ such that

$$
\log \phi_{n+1}(x)-\log \phi_{n}(T x) \rightarrow g(x)
$$

uniformly on $X$ as $n \rightarrow \infty$, then $\Phi$ is asymptotically additive.

(iv) $\Phi$ is asymptotically additive if and only if for any $\epsilon>0$, there exists an additive potential $\Psi=\left\{\log \psi_{n}\right\}_{n=1}^{\infty}$ on $X$ such that

$$
\limsup _{n \rightarrow \infty} \frac{1}{n} \sup _{x \in X}\left|\log \phi_{n}(x)-\log \psi_{n}(x)\right| \leq \epsilon .
$$

Proof. To see (i), define $\Psi=\left\{\log \psi_{n}\right\}_{n=1}^{\infty}$ by $\psi_{n}(x)=C \phi_{n}(x)$. Then

$$
\psi_{n+m}(x)=C \phi_{n+m}(x) \leq C^{2} \phi_{n}(x) \phi_{m}(x)=\psi_{n}(X) \psi_{m}\left(T^{n} x\right),
$$

Hence $\Psi$ is sub-additive. Clearly, $\left(\log \psi_{n}(x)-\log \phi_{n}(x)\right) / n=(\log C) / n \rightarrow 0$ as $n \rightarrow \infty$. Hence $\Phi$ is asymptotically sub-additive. This proves (i). Part (ii) follows directly from (i).

To show (iii), define $r_{n}=\sup _{x \in X}\left|\log \phi_{n}(x)-\log \phi_{n-1}(T x)-g(x)\right|$, with the convention $\log \phi_{0}(x) \equiv 0$. It is clear that $\lim _{n \rightarrow \infty} r_{n}=0$. Let $g_{n}=\sum_{i=0}^{n-1} g \circ T^{i}$. Then $\mathcal{G}=\left\{g_{n}\right\}_{n=1}^{\infty}$ is additive. Note that

$$
\begin{aligned}
\left|\log \phi_{n}(x)-g_{n}(x)\right| & =\left|\sum_{i=1}^{n}\left(\log \phi_{i}\left(T^{n-i} x\right)-\log \phi_{i-1}\left(T^{n-i+1} x\right)-g\left(T^{n-i} x\right)\right)\right| \\
& \leq \sum_{i=1}^{n}\left|\log \phi_{i}\left(T^{n-i} x\right)-\log \phi_{i-1}\left(T^{n-i+1} x\right)-g\left(T^{n-i} x\right)\right| \leq \sum_{i=1}^{n} r_{i} .
\end{aligned}
$$

Hence $\lim \sup _{n \rightarrow \infty} \sup _{x \in X}\left|\frac{\log \phi_{n}(x)-g_{n}(x)}{n}\right| \leq \lim \sup _{n \rightarrow \infty} \frac{1}{n} \sum_{i=1}^{n} r_{i}=0$ as $\lim _{n \rightarrow+\infty} r_{n}=0$. Hence $\Phi$ is asymptotically additive.

The "if" part in (iv) is direct, we only need to show the "only if" part. Assume that $\Phi$ is asymptotically additive, that is, $\phi_{n}$ is positive continuous on $X$ for each $n$ and both 
$\left\{\log \phi_{n}\right\}_{n=1}^{\infty}$ and $\left\{\log \left(\phi_{n}\right)^{-1}\right\}_{n=1}^{\infty}$ are asymptotically sub-additive. We claim that for any $\epsilon>0$, there exists $K>0$ such that for each $k \geq K$, there exists $C_{\epsilon, k}>0$ so that

$$
\left|\log \phi_{n}(x)-\frac{1}{k} \sum_{j=0}^{n-1} \log \phi_{k}\left(T^{j} x\right)\right| \leq n \epsilon+C_{\epsilon, k}, \quad \forall n \geq 2 k, x \in X,
$$

Clearly the above inequality implies the "only if" part in (iv). Without loss of generality, we show that

$$
\log \phi_{n}(x) \leq \frac{1}{k} \sum_{j=0}^{n-1} \log \phi_{k}\left(T^{j} x\right)+n \epsilon+C_{\epsilon, k}, \quad \forall n \geq 2 k, x \in X .
$$

for certain $C_{\epsilon, k}>0$. Fix $\epsilon>0$. Since $\Phi$ is asymptotically sub-additive, there exists a sub-additive potential $\Psi=\left\{\log \psi_{n}\right\}_{n=1}^{\infty}$ on $X$ such that there is $K>0$ and

$$
\left|\log \phi_{n}(x)-\log \psi_{n}(x)\right| \leq \frac{n \epsilon}{2}, \quad \forall n \geq K, x \in X
$$

Set $C=\max \left\{1, \sup _{x \in X} \psi_{1}(x)\right\}$. By [15, Lemma 2.2 ],

$$
\log \psi_{n}(x) \leq 2 k \log C+\frac{1}{k} \sum_{i=0}^{n-k} \log \psi_{k}\left(T^{i} x\right), \quad \forall x \in X, n \geq 2 k .
$$

Combining the above inequality with (A.4), we have for $k \geq K$,

$$
\begin{aligned}
\log \phi_{n}(x) & \leq(2 n-k) \epsilon / 2+2 k \log C+\frac{1}{k} \sum_{i=0}^{n-k} \log \phi_{k}\left(T^{i} x\right) \\
& \leq(2 n-k) \epsilon / 2+2 k \log C+M_{k}+\frac{1}{k} \sum_{i=0}^{n-1} \log \phi_{k}\left(T^{i} x\right)
\end{aligned}
$$

for all $x \in X$ and $n \geq 2 k$, where $M_{k}:=\max \left\{1, \sup _{x \in X}\left|\log \phi_{k}(x)\right|\right\}$. This proves (A.3), with $C_{\epsilon, k}=2 k \log C+M_{k}$. We finish the proof of the proposition.

Remark A.6. (i) The potentials satisfying the assumption in Proposition A.5(iii) was considered by Barreira [3] in the study of the Hausdorff dimension of planar limit sets.

(ii) Let $\mathcal{C}_{a a}(X, T)$ denote the collection of asymptotically additive potentials on $X$. Define an equivalence relation $\sim$ on $\mathcal{C}_{a a}(X, T)$ by $\Phi \sim \Psi$ if $\|\Phi-\Psi\|_{\lim }=0$, where

$$
\|\Phi-\Psi\|_{\lim }:=\limsup _{n \rightarrow \infty} \frac{1}{n} \sup _{x \in X}\left|\log \phi_{n}(x)-\log \psi_{n}(x)\right|
$$

for $\Phi=\left\{\log \phi_{n}\right\}_{n=1}^{\infty}, \Psi=\left\{\log \psi_{n}\right\}_{n=1}^{\infty}$. Then it is not hard to see that the quotient space $\mathcal{C}_{a a}(X, T) / \sim$ endowed with the norm $\|\cdot\|_{\text {lim }}$ is a separable Banach space. 
TABLE 1. Main notation and conventions

\begin{tabular}{|c|c|}
\hline$(X, T)$ & A topological dynamical system (Section 1) \\
\hline$\Phi=\left\{\log \phi_{n}\right\}_{n=1}^{\infty}$ & (Asymptotically sub-additive) potential (Section 1) \\
\hline $\bar{\beta}(\Phi)$ & $\bar{\beta}(\Phi)=\lim _{n \rightarrow \infty}(1 / n) \log \sup _{x \in X} \phi_{n}(x)$ \\
\hline$\lambda_{\Phi}(x), \Phi_{*}(\mu)$ & Lyapunov exponent of $\Phi$ at $x$ (resp. with respect to $\mu$ ) (Section 1) \\
\hline$E_{\Phi}(\alpha)$ & $\alpha$-level set of $\lambda_{\Phi}$ (Section 1) \\
\hline $\mathcal{M}(X)$ & Set of all Borel probability measures on $X$ \\
\hline $\mathcal{M}(X, T), \mathcal{E}(X, T)$ & Set of $T$-invariant (resp. ergodic) Borel probability measures on $X$ \\
\hline$h_{\mu}(T)$ & measure-theoretic entropy of $T$ with respect to $\mu$ \\
\hline$P(T, \Phi)$ & Topological pressure of $\Phi$ (Section 2.2) \\
\hline$P_{\Phi}(q)$ & $P(T, q \Phi)$ \\
\hline$P_{\Phi}^{\prime}( \pm \infty)$ & $\lim _{q \rightarrow \infty} P_{\Phi}(q) / q, \lim _{q \rightarrow-\infty} P_{\Phi}(q) / q$ \\
\hline $\mathcal{I}(\Phi, q)$ & Set of equilibrium states of $q \Phi$ \\
\hline $\begin{array}{l}h_{\mathrm{top}}(T, Z), h_{\mathrm{top}}(T) \\
\operatorname{conv}(M)\end{array}$ & $\begin{array}{l}\text { Topological entropy of } T \text { with respect to } Z \text { (resp. } Z=X) \text { (Section 2.1) } \\
\text { Convex hull of } M \text { (Section } \sqrt{2.3})\end{array}$ \\
\hline $\operatorname{ri}(A)$ & Relative interior of a convex set $A$ \\
\hline$f^{*}$ & Conjugate function of $f$ (Section 2.4) \\
\hline $\operatorname{ext}(C), \operatorname{expo}(C)$ & Set of extreme points (resp. exposed points) of $C$ (Section 2.3) \\
\hline $\begin{array}{l}\partial f(\mathbf{x}) \\
\partial^{e} f(\mathbf{x})\end{array}$ & $\begin{array}{l}\text { Subdifferential of } f \text { at } \mathbf{x} \text { (Section } 2.3 \text { ) } \\
\operatorname{ext}(\partial f(\mathbf{x}))\end{array}$ \\
\hline$\partial f(U), \partial^{e} f(U)$ & $\bigcup_{\mathbf{x} \in U} \partial f(\mathbf{x}), \bigcup_{\mathbf{x} \in U} \partial^{e} f(\mathbf{x})$ \\
\hline$V(x)$ & Set of limit points of the sequence $\mu_{x, n}=(1 / n) \sum_{j=0}^{n-1} \delta_{T^{j} x}$ in $\mathcal{M}(X)$ \\
\hline $\mathbb{R}_{+}$ & $(0, \infty)$ \\
\hline $\mathrm{cl}_{+}(A)$ & (cf. (4.5)) \\
\hline $\mathrm{cl}_{+}^{\delta}\left(\partial P_{\boldsymbol{\Phi}}\left(\mathbb{R}_{+}^{k}\right)\right)$ & (cf. 4.22) \\
\hline$\underline{\Phi}=\left(\Phi_{1}, \ldots, \Phi_{k}\right)$ & A family of asymptotically sub-additive potentials \\
\hline $\bar{\beta}(\boldsymbol{\Phi})$ & $\bar{\beta}\left(\sum_{i=1}^{k} \Phi_{i}\right)$ \\
\hline $\mathbf{\Phi}_{*}(\mu)$ & $\left(\left(\Phi_{1}\right)_{*}(\mu), \ldots,\left(\Phi_{k}\right)_{*}(\mu)\right)$ \\
\hline$P_{\mathbf{\Phi}}(\mathbf{q})$ & $P(T, \mathbf{q} \cdot \mathbf{\Phi})$ \\
\hline $\mathcal{I}(\mathbf{\Phi}, \mathbf{q})$ & Set of equilibrium states of $\mathbf{q} \cdot \boldsymbol{\Phi}$ \\
\hline$E_{\mathbf{\Phi}}(\mathbf{a})$ & (cf. (4.4) $)$ \\
\hline$G_{\mu}$ & Set of $\mu$-generic points (see Section [5) \\
\hline
\end{tabular}

\section{Appendix B. Main notation and COnVEntions}

For the reader's convenience, we summarize in Table 1 the main notation and typographical conventions used in this paper.

Acknowledgements The first author was partially supported by the direct grant and RGC grants in the Hong Kong Special Administrative Region, China (projects CUHK400706, CUHK401008). The second author was partially supported by NSFC (Grant 10531010), 973 project and FANEDD (Grant 200520). The authors thank Yongluo Cao and Katrin Gelfert for their valuable comments. They also thank the anonymous referee for his helpful comments and suggestions that improved the manuscript. 


\section{REFERENCES}

[1] J. Barral and M. Mensi, Gibbs measures on self-affine Sierpinski carpets and their singularity spectrum. Ergod. Th. \& Dynam. Sys. 27 (2007), 1419-1443.

[2] J. Barral, and M. Mensi, Multifractal analysis of Birkhoff averages on 'self-affine' symbolic spaces. Nonlinearity 21 (2008), 2409-2425.

[3] L. Barreira, A non-additive thermodynamic formalism and applications to dimension theory of hyperbolic dynamical systems. Ergod. Th. E Dynam. Sys. 16 (1996), no. 5, 871-927.

[4] L. Barreira, Nonadditive thermodynamic formalism: equilibrium and Gibbs measures. Discrete Contin. Dyn. Syst., 16 (2006), 279-305.

[5] L. Barreira, Dimension and recurrence in hyperbolic dynamics. Progress in Mathematics, 272. Birkhäuser Verlag, Basel, 2008.

[6] L. Barreira and K. Gelfert, Multifractal analysis for Lyapunov exponents on nonconformal repellers. Comm. Math. Phys. 267 (2006), 393-418.

[7] L. Barreira, Ya. Pesin and J. Schmeling, On a general concept of multifractality: multifractal spectra for dimensions, entropies, and Lyapunov exponents. Multifractal rigidity. Chaos 7 (1997), 27-38.

[8] L. Barreira, B. Saussol and J. Schmeling, Higher-dimensional multifractal analysis. J. Math. Pures Appl. 81 (2002), 67-91.

[9] L. Barreira and J. Schmeling, Sets of "non-typical" points have full topological entropy and full Hausdorff dimension, Israel J. Math. 116 (2000), 29-70.

[10] F. Ben Nasr, Analyse multifractale de mesures. C. R. Acad. Sci. Paris Sér. I Math. 319 (1994), 807-810.

[11] R. Bowen, Entropy-expansive maps. Trans. Amer. Math. Soc. 164 (1972), 323-331.

[12] R. Bowen, Topological entropy for noncompact sets. Trans. Amer. Math. Soc. 184 (1973), 125-136.

[13] G. Brown, G. Michon and J. Peyrière, On the multifractal analysis of measures. J. Statist. Phys. 66 (1992), 775-790.

[14] J. Buzzi, Intrinsic ergodicity of smooth interval maps. Israel J. Math. 100 (1997), 125-161.

[15] Y. L. Cao, D. J. Feng and W. Huang, The thermodynamical formalism for submultiplicative potentials. Discrete Contin. Dyn. Syst. 20 (2008), 639-657.

[16] E. C. Chen, T. Küpper and L. Shu, Topological entropy for divergence points. Ergod. Th. E Dynam. Sys. 25 (2005), 1173-1208.

[17] J. B. Conway, A course in functional analysis. Springer-Verlag, New York, 1985.

[18] K. J. Falconer, A subadditive thermodynamic formalism for mixing repellers. J. Phys. A 21 (1988), no. 14, L737-L742.

[19] K. J. Falconer and A. Sloan, Continuity of subadditive pressure for self-affine sets. Real Analysis Exchange 34 (2008), no. 2, 413-428.

[20] A. H. Fan and D. J. Feng, On the distribution of long-term time averages on symbolic space. J. Statist. Phys. 99 (2000), 813-856.

[21] A. H. Fan, D. J. Feng and J. Wu, Recurrence, dimension and entropy, J. Lond. Math. Soc. 64 (2001), 229-244.

[22] A. H. Fan, L. M. Liao and J. Peyrière, Generic points in systems of specification and Banach valued Birkhoff ergodic average. Discrete Contin. Dyn. Syst. 21 (2008), 1103-1128.

[23] D. J. Feng, Lyapunov exponents for products of matrices and multifractal analysis. Part I: Positive matrices. Israel J. Math. 138 (2003), 353-376.

[24] D. J. Feng, Lyapunov exponents for products of matrices and multifractal analysis. Part II. General matrices. Israel J. Math. 170 (2009), 355-394.

[25] D. J. Feng, The variational principle for products of non-negative matrices. Nonlinearity 17 (2004), 447-457.

[26] D. J. Feng and K. S. Lau, The pressure function for products of non-negative matrices. Math. Res. Lett. 9 (2002), 363-378.

[27] D. J. Feng, K. S. Lau and J. Wu, Ergodic limits on the conformal repeller. Adv. Math. 169 (2002) 58-91. 
[28] D. J. Feng and E. Olivier, Multifractal analysis of the weak Gibbs measures and phase transitionapplication to some Bernoulli convolutions. Ergod. Th. ES Dynam. Sys., 23 (2003), 1751-1784.

[29] D. J. Feng and L. Shu, Multifractal analysis for disintegrations of Gibbs measures and conditional Birkhoff averages. Ergod. Th. ES Dynam. Sys., 29 (2009), 885-918.

[30] J. P Hiriart-Urruty and C. Lemaréchal, Fundamentals of convex analysis. Springer-Verlag, Berlin, 2001.

[31] A. Käenmäki. On natural invariant measures on generalised iterated function systems. Ann. Acad. Sci. Fenn. Math., 29 (2004), 419-458.

[32] A. Katok and B. Hasselblatt, Introduction to the modern theory of dynamical systems. Cambridge University Press, Cambridge, 1995.

[33] G. Keller, Equilibrium states in ergodic theory, Cambridge University Press, 1998.

[34] M. Kesseböhmer, Large deviation for weak Gibbs measures and multifractal spectra. Nonlinearity 14 (2001), 395-409.

[35] M. Kesseböhmer and B. O. Stratmann, A multifractal formalism for growth rates and applications to geometrically finite Kleinian groups. Ergod. Th. ES Dynam. Sys. 24 (2004), 141-170.

[36] W. Krieger, On unique ergodicity. Proceedings of the Sixth Berkeley Symposium on Mathematical Statistics and Probability (Univ. California, Berkeley, Calif., 1970/1971), Vol. II: Probability theory, pp. 327-346, Univ. California Press, Berkeley, Calif., 1972.

[37] N. Makarov and S. Smirnov, On "thermodynamics" of rational maps. I. Negative spectrum. Comm. Math. Phys. 211 (2000), 705-743.

[38] N. Makarov and S. Smirnov, On thermodynamics of rational maps. II. Non-recurrent maps. J. London Math. Soc. 67 (2003), 417-432.

[39] M. Misiurewicz, Topological conditional entropy. Studia Math. 55 (1976), 175-200.

[40] A. Mummert, The thermodynamic formalism for almost-additive sequences. Discrete Contin. Dyn. Syst., 16 (2006), 435-454.

[41] E. Olivier, Multifractal analysis in symbolic dynamics and distribution of pointwise dimension for g-measures. Nonlinearity 12 (1999), 1571-1585.

[42] L. Olsen, Multifractal analysis of divergence points of deformed measure theoretical Birkhoff averages. J. Math. Pures Appl. 82 (2003), 1591-1649.

[43] Y. B. Pesin, Dimension theory in dynamical systems. Contemporary views and applications. Chicago Lectures in Mathematics. University of Chicago Press, Chicago, IL, 1997.

[44] C.-E. Pfister, and W. G. Sullivan, On the topological entropy of saturated sets. Ergod. Th. ES Dynam. Sys. 27 (2007), 929-956.

[45] R. T. Rockafellar, Convex analysis. Princeton University Press, Princeton, N.J. 1970.

[46] D. Ruelle, Thermodynamic formalism. The mathematical structures of classical equilibrium statistical mechanics. Encyclopedia of Mathematics and its Applications, 5. Addison-Wesley Publishing Co., Reading, Mass., 1978.

[47] F. Takens and E. Verbitskiy, On the variational principle for the topological entropy of certain noncompact sets. Ergod. Th. \& Dynam. Sys. 23 (2003), 317-348.

[48] B. Testud, Phase transitions for the multifractal analysis of self-similar measures. Nonlinearity 19 (2006), 1201-1217.

[49] P. Walters, An introduction to ergodic theory, Springer-Verlag, Berlin, Heidelberg, New York, 1982.

Department of Mathematics, The Chinese University of Hong Kong, Shatin, Hong Kong,

E-mail address: djfeng@math.cuhk.edu.hk

Department of Mathematics, University of Science and Technology of China, Hefei 230026 , Anhui, P. R. China,

E-mail address: wenh@mail.ustc.edu.cn 